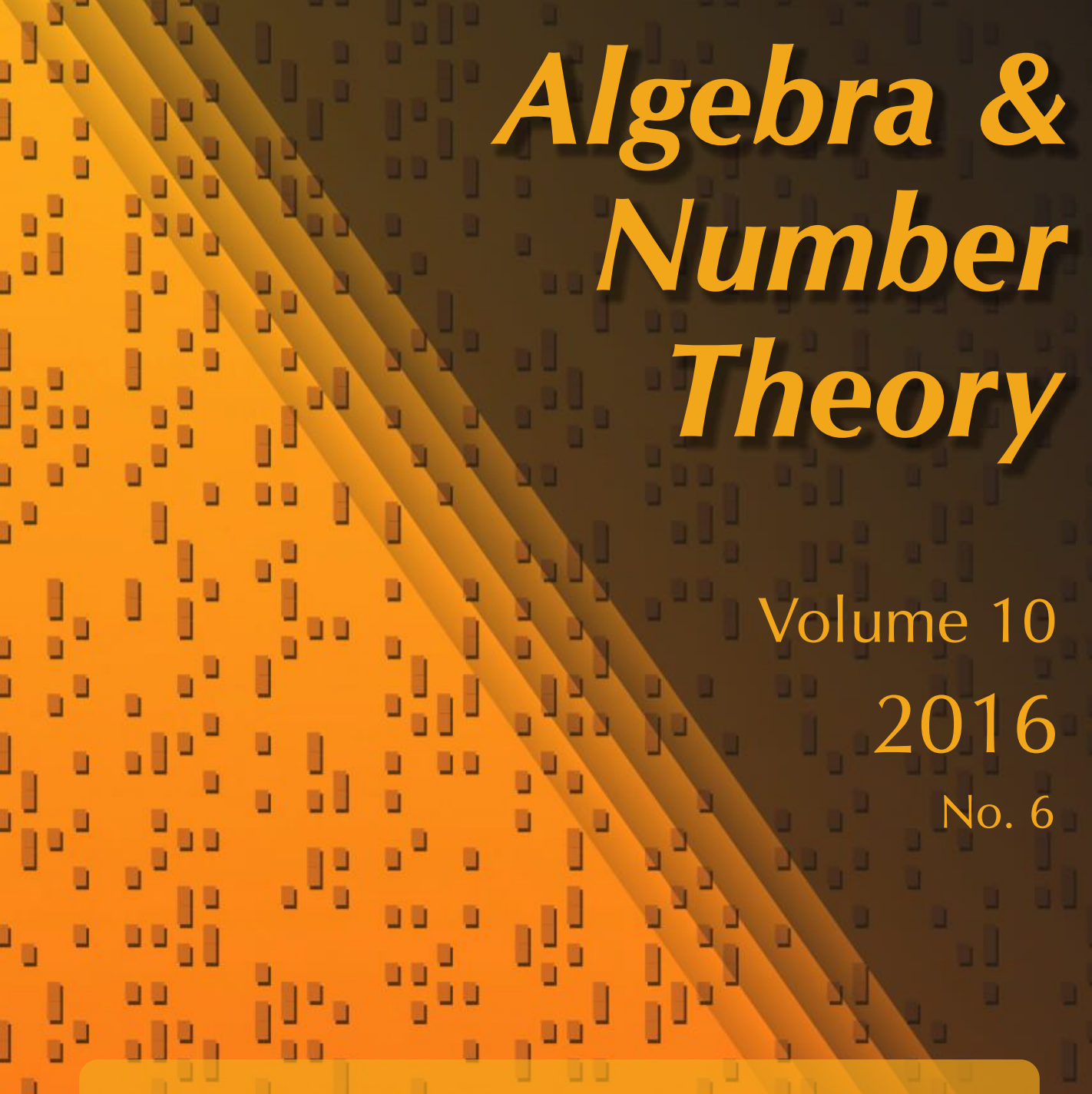

On the local Tamagawa number conjecture for Tate motives over tamely ramified fields

Jay Daigle and Matthias Flach

J.

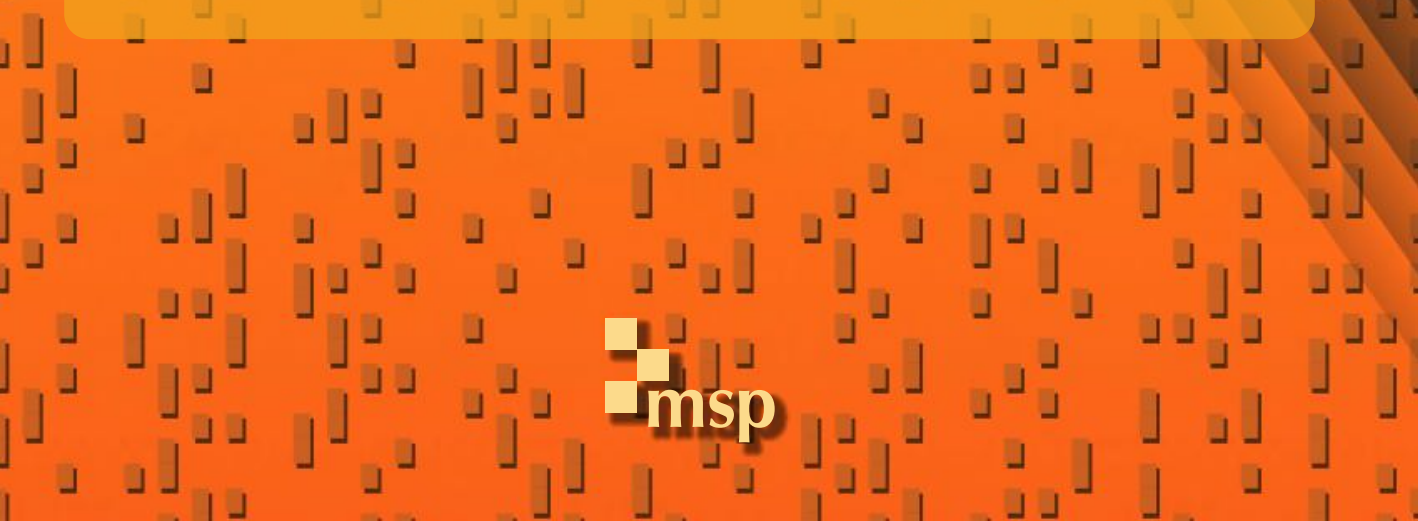




\title{
On the local Tamagawa number conjecture for Tate motives over tamely ramified fields
}

\author{
Jay Daigle and Matthias Flach
}

\begin{abstract}
The local Tamagawa number conjecture, which was first formulated by Fontaine and Perrin-Riou, expresses the compatibility of the (global) Tamagawa number conjecture on motivic L-functions with the functional equation. The local conjecture was proven for Tate motives over finite unramified extensions $K / \mathbb{Q}_{p}$ by Bloch and Kato. We use the theory of $(\varphi, \Gamma)$-modules and a reciprocity law due to Cherbonnier and Colmez to provide a new proof in the case of unramified extensions, and to prove the conjecture for $\mathbb{Q}_{p}(2)$ over certain tamely ramified extensions.
\end{abstract}

\section{Introduction}

Let $K / \mathbb{Q}_{p}$ be a finite extension and $V$ a de Rham representation of $G_{K}:=\operatorname{Gal}(\bar{K} / K)$. The local Tamagawa number conjecture is a statement describing a certain $\mathbb{Q}_{p}$-basis of the determinant line $\operatorname{det}_{\mathbb{Q}_{p}} R \Gamma(K, V)$ of (continuous) local Galois cohomology up to units in $\mathbb{Z}_{p}^{\times}$. It was first formulated by Fontaine and Perrin-Riou [1994, 4.5.4] as conjecture $C_{\mathrm{EP}}$ and independently by Kato [1993, Conjecture 1.8] as the "local $\epsilon$-conjecture". Both conjectures express compatibility of the (global) Tamagawa number conjecture on motivic L-functions with the functional equation. The fact that the local Tamagawa number conjecture is equivalent to this compatibility still constitutes its main interest. For example, the proof of the Tamagawa number conjecture for Dirichlet L-functions at integers $r \geq 2$ [Burns and Flach 2006] uses the conjecture at $1-r$ and compatibility with the functional equation (no other more direct proof is known). Fukaya and Kato [2006] generalized [Kato 1993, Conjecture 1.8] to de Rham representations with coefficients in a possibly noncommutative $\mathbb{Q}_{p}$-algebra, and in fact to arbitrary $p$-adic families of local Galois representations.

In this paper we shall only consider Tate motives $V=\mathbb{Q}_{p}(r)$ with $r \geq 2$ (for the case $r=1$ see [Bley and Cobbe 2016; Breuning 2004]). If $K / \mathbb{Q}_{p}$ is unramified the local Tamagawa number conjecture for $\mathbb{Q}_{p}(r)$ was first proven by Bloch and

MSC2010: primary 14F20; secondary 11G40, 18F10, 22A99.

Keywords: Tamagawa number conjecture. 
Kato [1990] in their seminal paper on the global Tamagawa number conjecture, and has since been reproven by a number of authors (e.g., [Perrin-Riou 1994; Benois and Berger 2008]). These later proofs also cover the case where $K / \mathbb{Q} p$ is a cyclotomic extension, or more generally where $V$ is an abelian de Rham representations of $\operatorname{Gal}\left(\overline{\mathbb{Q}}_{p} / \mathbb{Q}_{p}\right)$ [Kato 1993, Theorem 4.1; Venjakob 2013]. All proofs have two main ingredients: Iwasawa theory and a "reciprocity law". The latter is an explicit description of the exponential or dual exponential map for the de Rham representation $V$, which however very often only holds in restricted situations (e.g., $V$ ordinary or absolutely crystalline). The aim of this paper is to explore the application of the very general reciprocity law of Cherbonnier and Colmez [1999], which holds for arbitrary de Rham representations, to the local Tamagawa number conjecture for Tate motives.

In Section 2 we give a first somewhat explicit statement (Proposition 2) which is equivalent to the local Tamagawa conjecture for $\mathbb{Q}_{p}(r)$ over an arbitrary Galois extension $K / \mathbb{Q}_{p}$. We in fact work with the refined equivariant conjecture over the group ring $\mathbb{Z}_{p}\left[\mathrm{Gal}\left(K / \mathbb{Q}_{p}\right)\right]$, following Fukaya and Kato [2006]. In Section 3 we focus on the case where $p \nmid\left[K: \mathbb{Q}_{p}\right]$. In Section 4 we state the reciprocity law of Cherbonnier and Colmez in the case of Tate motives. In Section 5 we show that it also can be used to give a proof of the unramified case (which however has many common ingredients with the existing proofs). Finally, in Section 6 we formulate our main result, Proposition 44, which is a fairly explicit statement equivalent to the equivariant local Tamagawa number conjecture for $\mathbb{Q}_{p}(r)$ over $K / \mathbb{Q}_{p}$ with $p \nmid\left[K: \mathbb{Q}_{p}\right]$. We show that it can be used to prove some new cases; more specifically we have:

Proposition 1. Assume $K / \mathbb{Q}_{p}$ is Galois of degree prime to $p$ and with ramification degree $e<p / 4$. Then the equivariant local Tamagawa number conjecture holds for $V=\mathbb{Q}_{p}(2)$.

The only cases where the conjecture for tamely ramified fields was known previously are cyclotomic fields, i.e., where $e \mid p-1$, and in this case one can allow arbitrary $r$ [Perrin-Riou 1994; Benois and Berger 2008]. We believe many more cases can be proven with Proposition 44 and hope to return to this in a subsequent article.

\section{The conjecture}

Throughout this paper $p$ denotes an odd prime. Let $K / \mathbb{Q}_{p}$ be an arbitrary finite Galois extension with group $G$ and $r \geq 2$. In this section we shall explicate the consequences of the local Tamagawa number conjecture of Fukaya and Kato [2006, Conjecture 3.4.3] for the triple

$$
(\Lambda, T, \zeta)=\left(\mathbb{Z}_{p}[G], \operatorname{Ind}_{G_{K}}^{G_{\mathbb{Q}_{p}}} \mathbb{Z}_{p}(1-r), \zeta\right) .
$$


Here $\zeta=\left(\zeta_{p^{n}}\right)_{n} \in \Gamma\left(\overline{\mathbb{Q}}_{p}, \mathbb{Z}_{p}(1)\right)$ is a compatible system of $p^{n}$-th roots of unity which we fix throughout this paper. The conjectures for a triple $(\Lambda, T, \zeta)$ and its dual $\left(\Lambda^{\mathrm{op}}, T^{*}(1), \zeta\right)$ are equivalent. We find it advantageous to work with $\mathbb{Q}_{p}(1-r)$ rather than $\mathbb{Q}_{p}(r)$ as in [Bloch and Kato 1990] since we are employing the Cherbonnier-Colmez reciprocity law [Cherbonnier and Colmez 1999] which describes the dual exponential map.

In order to give an idea what the conjecture is about, consider the Bloch-Kato exponential map [Bloch and Kato 1990]

$$
\exp : K \stackrel{\sim}{\longrightarrow} H^{1}\left(K, \mathbb{Q}_{p}(r)\right) .
$$

In a first approximation one may say that the local Tamagawa number conjecture describes the relation between the two $\mathbb{Z}_{p}$-lattices $\exp \left(\mathcal{O}_{K}\right)$ and $\operatorname{im}\left(H^{1}\left(K, \mathbb{Z}_{p}(r)\right)\right)$ inside $H^{1}\left(K, \mathbb{Q}_{p}(r)\right)$. Rather than giving a complete description of the relative position of these two lattices, the conjecture only specifies their relative volume, that is the class in $\mathbb{Q}_{p}^{\times} / \mathbb{Z}_{p}^{\times}$which multiplies $\operatorname{Det}_{\mathbb{Z}_{p}} \exp \left(\mathcal{O}_{K}\right)$ to $\operatorname{Det}_{\mathbb{Z}_{p}}\left(\operatorname{im}\left(H^{1}\left(K, \mathbb{Z}_{p}(r)\right)\right)\right)$ inside the $\mathbb{Q}_{p}$-line $\operatorname{Det}_{\mathbb{Q}_{p}} H^{1}\left(K, \mathbb{Q}_{p}(r)\right)$. The equivariant form of the conjecture is a finer statement which arises by replacing determinants over $\mathbb{Z}_{p}$ by determinants over $\mathbb{Z}_{p}[G]$. If $G$ is abelian and $\operatorname{im}\left(H^{1}\left(K, \mathbb{Z}_{p}(r)\right)\right)$ is projective over $\mathbb{Z}_{p}[G]$, the conjecture thereby does specify the relative position of the two lattices in view of the fact that $H^{1}\left(K, \mathbb{Q}_{p}(r)\right)$ is free of rank one over $\mathbb{Q}_{p}[G]$ and so coincides with its determinant. If $G$ is nonabelian, even though $H^{1}\left(K, \mathbb{Q}_{p}(r)\right)$ remains free of rank one over $\mathbb{Q}_{p}[G]$, the conjecture is an identity in the algebraic K-group $\left.\left.K_{1}\left(\mathbb{Q}_{p}^{\text {ur }}[G]\right)\right) / K_{1}\left(\mathbb{Z}_{p}^{\text {ur }}[G]\right)\right)$ and is again quite a bit weaker than a full determination of the relative position of the two lattices.

Determinants in the sense of [Deligne 1987] (see also [Fukaya and Kato 2006, 1.2]) are only defined for modules of finite projective dimension, or more generally perfect complexes, and so the first step is to replace the $\mathbb{Z}_{p}$-lattice $\operatorname{im}\left(H^{1}\left(K, \mathbb{Z}_{p}(r)\right)\right)$ by the entire perfect complex $R \Gamma\left(K, \mathbb{Z}_{p}(r)\right)$. There still is an isomorphism

$$
R \Gamma\left(K, \mathbb{Z}_{p}(r)\right) \otimes_{\mathbb{Z}_{p}} \mathbb{Q}_{p} \cong R \Gamma\left(K, \mathbb{Q}_{p}(r)\right) \cong H^{1}\left(K, \mathbb{Q}_{p}(r)\right)[-1]
$$

since the groups $H^{1}\left(K, \mathbb{Z}_{p}(r)\right)_{\text {tor }}$ and $H^{2}\left(K, \mathbb{Z}_{p}(r)\right)$ are finite. If $K / \mathbb{Q}_{p}$ is Galois with group $G$ then $R \Gamma\left(K, \mathbb{Z}_{p}(r)\right)$ is always a perfect complex of $\mathbb{Z}_{p}[G]$-modules whereas $\operatorname{im}\left(H^{1}\left(K, \mathbb{Z}_{p}(r)\right)\right)$ or $\mathcal{O}_{K}$ need no longer have finite projective dimension over $\mathbb{Z}_{p}[G]$. A further simplification occurs if one does not try to compare $R \Gamma\left(K, \mathbb{Z}_{p}(r)\right)$ to $\exp \left(\mathcal{O}_{K}\right)$ directly. Instead one uses the "period isomorphism"

$$
\text { per : } \overline{\mathbb{Q}}_{p} \otimes_{\mathbb{Q}_{p}} K \cong \overline{\mathbb{Q}}_{p} \otimes_{\mathbb{Q}_{p}}\left(\operatorname{Ind}_{G_{K}}^{G_{\mathbb{Q}_{p}}} \mathbb{Q}_{p}\right) \cong \overline{\mathbb{Q}}_{p}[G]
$$

and tries to compare $\operatorname{Det}_{\mathbb{Z}_{p}} R \Gamma\left(K, \mathbb{Z}_{p}(r)\right)$ to a suitable lattice in this last space. The left- $\mathbb{Z}_{p}[G]$-module $\operatorname{Ind}_{G_{K}}^{G_{\mathbb{Q}_{p}}} \mathbb{Z}_{p}$ is always free of rank one whereas $\mathcal{O}_{K}$ need not be. After choosing an embedding $K \rightarrow \overline{\mathbb{Q}}_{p}$ one gets an isomorphism $\psi: G_{\mathbb{Q}_{p}} / G_{K} \cong G$ 
and an isomorphism

$$
\operatorname{Ind}_{G_{K}}^{G_{Q_{p}}} \mathbb{Z}_{p} \cong \mathbb{Z}_{p}[G]
$$

so that the $\mathbb{Z}_{p}[G]$-linear left action of $\gamma \in G_{\mathbb{Q}_{p}}$ is given by

$$
\mathbb{Z}_{p}[G] \ni x \mapsto x \psi\left(\gamma^{-1}\right) .
$$

The period isomorphism is then given for $x \in K$ by

$$
\operatorname{per}(x):=\operatorname{per}(1 \otimes x)=\sum_{g \in G} g(x) \cdot g^{-1} \in \overline{\mathbb{Q}}_{p}[G] .
$$

The dual of exp identifies with the dual exponential map

$$
\exp _{\mathbb{Q}_{p}(r)}^{*}: H^{1}\left(K, \mathbb{Q}_{p}(1-r)\right) \rightarrow K
$$

by local Tate duality and the trace pairing on $K$. Let $\beta \in H^{1}\left(K, \mathbb{Z}_{p}(1-r)\right)$ be an element spanning a free $\mathbb{Z}_{p}[G]$-submodule and let $C_{\beta}$ be the mapping cone of the ensuing map of perfect complexes of $\mathbb{Z}_{p}[G]$-modules

$$
\left(\mathbb{Z}_{p}[G] \cdot \beta\right)[-1] \rightarrow H^{1}\left(K, \mathbb{Z}_{p}(1-r)\right)[-1] \rightarrow R \Gamma\left(K, \mathbb{Z}_{p}(1-r)\right) .
$$

Then $C_{\beta}$ is a perfect complex of $\mathbb{Z}_{p}[G]$-modules with finite cohomology groups, i.e., such that $C_{\beta} \otimes_{\mathbb{Z}_{p}} \mathbb{Q}_{p}$ is acyclic. It therefore represents a class $\left[C_{\beta}\right]$ in the relative $K$-group $K_{0}\left(\mathbb{Z}_{p}[G], \mathbb{Q}_{p}\right)$ for which one has an exact sequence

$$
K_{1}\left(\mathbb{Z}_{p}[G]\right) \rightarrow K_{1}\left(\mathbb{Q}_{p}[G]\right) \rightarrow K_{0}\left(\mathbb{Z}_{p}[G], \mathbb{Q}_{p}\right) \rightarrow 0 .
$$

Hence we may also view $\left[C_{\beta}\right]$ as an element in $K_{1}\left(\mathbb{Q}_{p}[G]\right) / \operatorname{im}\left(K_{1}\left(\mathbb{Z}_{p}[G]\right)\right)$. Extending scalars to $\overline{\mathbb{Q}}_{p}$ we get an isomorphism of free rank-one $\overline{\mathbb{Q}}_{p}[G]$-modules

$$
H^{1}\left(K, \mathbb{Q}_{p}(1-r)\right) \otimes_{\mathbb{Q}_{p}} \overline{\mathbb{Q}}_{p} \stackrel{\exp ^{*} \otimes \overline{\mathbb{Q}}_{p}}{\longrightarrow} K \otimes_{\mathbb{Q}_{p}} \overline{\mathbb{Q}}_{p} \stackrel{\text { per }}{\longrightarrow} \overline{\mathbb{Q}}_{p}[G]
$$

sending the $\overline{\mathbb{Q}}_{p}[G]$-basis $\beta$ to a unit $\operatorname{per}\left(\exp ^{*}(\beta)\right) \in \overline{\mathbb{Q}}_{p}[G]^{\times}$. As such it has a class

$$
\left[\operatorname{per}\left(\exp ^{*}(\beta)\right)\right] \in K_{1}\left(\overline{\mathbb{Q}}_{p}[G]\right)
$$

via the natural projection map $\overline{\mathbb{Q}}_{p}[G]^{\times} \rightarrow K_{1}\left(\overline{\mathbb{Q}}_{p}[G]\right.$ ) (recall that for any ring $R$ we have maps $\left.R^{\times} \rightarrow \mathrm{GL}(R) \rightarrow \mathrm{GL}(R)^{\mathrm{ab}}=: K_{1}(R)\right)$. In Section 2.2 below we shall define an $\epsilon$-factor $\epsilon\left(K / \mathbb{Q}_{p}, 1-r\right) \in K_{1}\left(\overline{\mathbb{Q}}_{p}[G]\right)$ such that

$$
\epsilon\left(K / \mathbb{Q}_{p}, 1-r\right) \cdot\left[\operatorname{per}\left(\exp ^{*}(\beta)\right)\right] \in K_{1}\left(\mathbb{Q}_{p}^{\mathrm{ur}}[G]\right) .
$$

Let $F \subseteq K$ denote the maximal unramified subfield, $\Sigma=\operatorname{Gal}\left(F / \mathbb{Q}_{p}\right)$ and $\sigma \in \Sigma$ the (arithmetic) Frobenius automorphism. Then $\mathbb{Q}_{p}[\Sigma]$ is canonically a direct factor of $\mathbb{Q}_{p}[G]$ and $\mathbb{Q}_{p}[\Sigma]^{\times} \cong K_{1}\left(\mathbb{Q}_{p}[\Sigma]\right)$ a direct factor of $K_{1}\left(\mathbb{Q}_{p}[G]\right)$. For $\alpha \in \mathbb{Q}_{p}[\Sigma]^{\times}$ we denote by $[\alpha]_{F}$ its class in $K_{1}\left(\mathbb{Q}_{p}[G]\right)$. Finally, note that if $R$ is a $\mathbb{Q}$-algebra then any nonzero rational number $n$ has a class $[n] \in K_{1}(R)$ via $\mathbb{Q}^{\times} \rightarrow R^{\times} \rightarrow K_{1}(R)$. 
Proposition 2. Let $K / \mathbb{Q}_{p}$ be Galois with group $G$ and $r \geq 2$. The local Tamagawa number conjecture for the triple

$$
(\Lambda, T, \zeta)=\left(\mathbb{Z}_{p}[G], \operatorname{Ind}_{G_{K}}^{G_{\mathbb{Q}_{p}}} \mathbb{Z}_{p}(1-r), \zeta\right)
$$

is equivalent to the identity

$$
[(r-1) !] \cdot \epsilon\left(K / \mathbb{Q}_{p}, 1-r\right) \cdot\left[\operatorname{per}\left(\exp ^{*}(\beta)\right)\right] \cdot\left[C_{\beta}\right]^{-1} \cdot\left[\frac{1-p^{r-1} \sigma}{1-p^{-r} \sigma^{-1}}\right]_{F}=1
$$

in the group $K_{1}\left(\mathbb{Q}_{p}^{\mathrm{ur}}[G]\right) / \operatorname{im}\left(K_{1}\left(\mathbb{Z}_{p}^{\mathrm{ur}}[G]\right)\right)$.

Before we begin the proof of the proposition we explain what we mean by the local Tamagawa number conjecture for $\left(\mathbb{Z}_{p}[G], \operatorname{Ind}_{G_{K}}^{G_{\mathbb{Q}_{p}}} \mathbb{Z}_{p}(1-r), \zeta\right)$. The local Tamagawa number conjecture [Fukaya and Kato 2006, Conjecture 3.4.3] claims the existence of $\epsilon$-isomorphisms $\epsilon_{\Lambda, \zeta}(T)$ for all triples $(\Lambda, T, \zeta)$, where $\Lambda$ is a semilocal pro- $p$ ring satisfying a certain finiteness condition [Fukaya and Kato 2006, 1.4.1], $T$ a finitely generated projective $\Lambda$-module with continuous $G_{\mathbb{Q}_{p}}$-action and $\zeta$ a basis of $\Gamma\left(\overline{\mathbb{Q}}_{p}, \mathbb{Z}_{p}(1)\right)$, such that certain functorial properties hold. One of these properties [Fukaya and Kato 2006, Conjecture 3.4.3(v)] says that if $L:=\Lambda \otimes_{\mathbb{Z}_{p}} \mathbb{Q}_{p}$ is a finite extension of $\mathbb{Q}_{p}$ and $V:=T \otimes_{\mathbb{Z}_{p}} \mathbb{Q}_{p}$ is a de Rham representation, then

$$
\tilde{L} \otimes_{\tilde{\Lambda}} \epsilon_{\Lambda, \zeta}(T)=\epsilon_{L, \zeta}(V),
$$

where $\epsilon_{L, \zeta}(V)$ is the isomorphism in $C_{\tilde{L}}$ defined in [Fukaya and Kato 2006, 3.3]. Here, for any ring $R, C_{R}$ is the Picard category constructed in [Fukaya and Kato 2006, 1.2], equivalent to the category of virtual objects of [Deligne 1987], $S \otimes_{R}-$ : $C_{R} \rightarrow C_{S}$ is the Picard functor induced by a ring homomorphism $R \rightarrow S$ and $\widetilde{R}=W\left(\overline{\mathbb{F}}_{p}\right) \otimes_{\mathbb{Z}_{p}} R$ for any $\mathbb{Z}_{p}$-algebra $R$. The construction of $\epsilon_{L, \zeta}(V)$ involves certain isomorphisms and exact sequences which we recall in the proof below. If $A$ is a finite dimensional semisimple $\mathbb{Q}_{p}$-algebra and $V$ an $A$-linear de Rham representation, those isomorphisms and exact sequences are in fact $A$-linear and therefore lead to an isomorphism $\epsilon_{A, \zeta}(V)$ in the category $C_{\tilde{A}}$. If $A:=\Lambda \otimes_{\mathbb{Z}_{p}} \mathbb{Q}_{p}$ is a semisimple $\mathbb{Q}_{p}$-algebra and $V:=T \otimes_{\mathbb{Z}_{p}} \mathbb{Q}_{p}$ is a de Rham representation, we say that the local Tamagawa number conjecture holds for the particular triple $(\Lambda, T, \zeta)$ if

$$
\tilde{A} \otimes_{\tilde{\Lambda}} \epsilon_{\Lambda, \zeta}(T)=\epsilon_{A, \zeta}(V)
$$

for some isomorphism $\epsilon_{\Lambda, \zeta}(T)$ in $C_{\tilde{\Lambda}}$.

Proof of Proposition 2. For a perfect complex of $\mathbb{Q}_{p}[G]$-modules $P$, we set

$$
P^{*}=\operatorname{Hom}_{\mathbb{Q}_{p}[G]}\left(P, \mathbb{Q}_{p}[G]\right),
$$


which is a perfect complex of $\mathbb{Q}_{p}[G]^{\mathrm{op}}$-modules. Fix $r \geq 2$ and set

$$
V=\operatorname{Ind}_{G_{K}}^{G_{Q_{p}}} \mathbb{Q}_{p}(1-r) \quad \text { and } \quad V^{*}(1)=\operatorname{Ind}_{G_{K}}^{G_{\mathbb{Q}_{p}}} \mathbb{Q}_{p}(r),
$$

which are free of rank one over $\mathbb{Q}_{p}[G]$ and $\mathbb{Q}_{p}[G]^{\text {op }}$, respectively. We recall the ingredients of the isomorphism $\theta_{\mathbb{Q}_{p}[G]}(V)$ of [Fukaya and Kato 2006, 3.3.2] (or rather of its generalization from field coefficients to semisimple coefficients). The element $\zeta$ determines an element $t=\log (\zeta)$ of $B_{\mathrm{dR}}$. We have

$$
\begin{gathered}
D_{\text {cris }}(V)=F \cdot t^{r-1}, \quad D_{\mathrm{dR}}(V) / D_{\mathrm{dR}}^{0}(V)=0, \\
D_{\text {cris }}\left(V^{*}(1)\right)=F \cdot t^{-r}, \quad D_{\mathrm{dR}}\left(V^{*}(1)\right) / D_{\mathrm{dR}}^{0}\left(V^{*}(1)\right)=K, \\
C_{f}\left(\mathbb{Q}_{p}, V\right): F \stackrel{1-p^{r-1} \sigma}{\longrightarrow} F, \\
C_{f}\left(\mathbb{Q}_{p}, V^{*}(1)\right): F \stackrel{\left(1-p^{-r} \sigma, \subseteq\right)}{\longrightarrow} F \oplus K,
\end{gathered}
$$

and commutative diagrams

$$
\begin{gathered}
\operatorname{Det}_{\mathbb{Q}_{p}[G]}(0) \stackrel{\eta\left(\mathbb{Q}_{p}, V\right)}{\longrightarrow} \operatorname{Det}_{\mathbb{Q}_{p}[G]} C_{f}\left(\mathbb{Q}_{p}, V\right) \cdot \operatorname{Det}_{\mathbb{Q}_{p}[G]} D_{\mathrm{dR}}(V) / D_{\mathrm{dR}}^{0}(V) \\
\operatorname{Det}_{\mathbb{Q}_{p}[G]}(0) \stackrel{\eta^{\prime}\left(\mathbb{Q}_{p}, V\right)}{\longrightarrow} \operatorname{Det}_{\mathbb{Q}_{p}[G]}(0) \cdot \operatorname{Det}_{\mathbb{Q}_{p}[G]}(0)^{-1} \cdot \operatorname{Det}_{\mathbb{Q}_{p}[G]}(0) \\
\prod_{\left[1-p^{-r} \sigma^{-1}\right]_{F}} \\
\operatorname{Det}_{\mathbb{Q}_{p}[G]}(0) \stackrel{\eta\left(\mathbb{Q}_{p}, V^{*}(1)\right)^{*,-1}}{\longrightarrow} \begin{array}{l}
\operatorname{Det}_{\mathbb{Q}_{p}[G]} C_{f}\left(\mathbb{Q}_{p}, V^{*}(1)\right)^{*} \\
\left.\operatorname{Det}_{\mathbb{Q}_{p}[G]} D_{\mathrm{dR}}\left(V^{*}(1)\right) / D_{\mathrm{dR}^{\prime}}^{0}\left(V^{*}(1)\right)\right)^{*}
\end{array} \\
\operatorname{Det}_{\mathbb{Q}_{p}[G]}(0) \stackrel{\eta^{\prime}\left(\mathbb{Q}_{p}, V^{*}(1)\right)^{*,-1}}{\longrightarrow} \operatorname{Det}_{\mathbb{Q}_{p}[G]}(0) \cdot \operatorname{Det}_{\mathbb{Q}_{p}[G]}\left(K^{*}\right)^{-1} \cdot \operatorname{Det}_{\mathbb{Q}_{p}[G]}\left(K^{*}\right)
\end{gathered}
$$

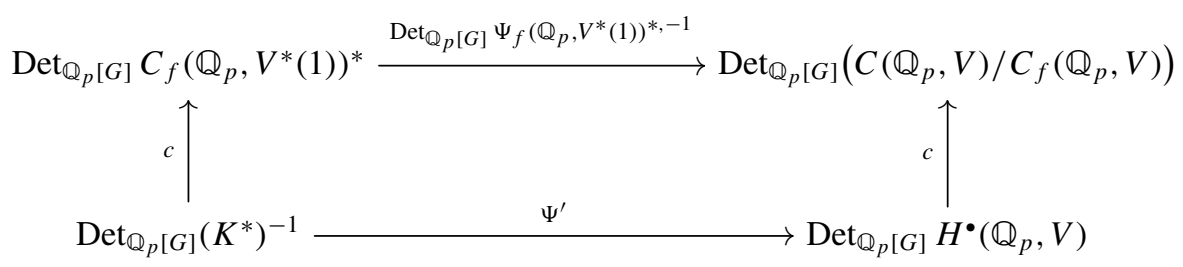

where the vertical maps $c$ are induced by passage to cohomology. The morphism $\Psi^{\prime}$ is $\left(\operatorname{Det}_{\mathbb{Q}_{p}[G]}^{-1}\right.$ of) the inverse of the isomorphism

$$
H^{1}\left(\mathbb{Q}_{p}, V\right) \stackrel{T}{\rightarrow} H^{1}\left(\mathbb{Q}_{p}, V^{*}(1)\right)^{*} \stackrel{\exp _{V^{*}(1)}^{*}}{\longrightarrow} K^{*},
$$

where $T$ is the local Tate duality isomorphism. For the isomorphism

$$
\theta_{\mathbb{Q}_{p}[G]}(V)=\eta\left(\mathbb{Q}_{p}, V\right) \cdot\left(\operatorname{Det}_{\mathbb{Q}_{p}[G]} \Psi_{f}\left(\mathbb{Q}_{p}, V^{*}(1)\right)^{*,-1} \circ \eta\left(\mathbb{Q}_{p}, V^{*}(1)\right)^{*,-1}\right)
$$


we obtain a commutative diagram

$$
\begin{gathered}
\operatorname{Det}_{\mathbb{Q}_{p}[G]}(0) \stackrel{\theta_{\mathbb{Q}_{p}[G]}(V)}{\longrightarrow} \operatorname{Det}_{\mathbb{Q}_{p}[G]} C\left(\mathbb{Q}_{p}, V\right) \cdot \operatorname{Det}_{\mathbb{Q}_{p}[G]} D_{\mathrm{dR}}(V) \\
{\left[\frac{1-p^{-r-1}}{1-p^{r-1} \sigma}\right]_{F} \uparrow} \\
\operatorname{Det}_{\mathbb{Q}_{p}[G]}(0) \stackrel{\theta^{\prime}}{\longrightarrow} \operatorname{Det}_{\mathbb{Q}_{p}[G]} H^{\bullet}\left(\mathbb{Q}_{p}, V\right) \cdot \operatorname{Det}_{\mathbb{Q}_{p}[G]}(K)
\end{gathered}
$$

where $\theta^{\prime}$ is induced by the dual exponential map

$$
H^{1}\left(\mathbb{Q}_{p}, V\right) \stackrel{\exp _{V^{*}(1)}^{*}}{\longrightarrow} K
$$

The isomorphism $\Gamma_{\mathbb{Q}_{p}[G]}(V) \cdot \epsilon_{\mathbb{Q}_{p}[G], \zeta, d R}(V)$ of [Fukaya and Kato 2006, 3.3.3] is the isomorphism

$$
\left[(-1)^{r-1}(r-1) !\right] \cdot \epsilon\left(K / \mathbb{Q}_{p}, 1-r\right) \cdot \operatorname{Det}_{\overline{\mathbb{Q}}_{p}[G]}(\text { per })
$$

and the isomorphism

$$
\epsilon_{\mathbb{Q}_{p}[G], \zeta}(V)=\Gamma_{\mathbb{Q}_{p}[G]}(V) \cdot \epsilon_{\mathbb{Q}_{p}[G], \zeta, d R}(V) \cdot \theta_{\mathbb{Q}_{p}[G]}(V)
$$

fits into a commutative diagram

$$
\begin{aligned}
& \operatorname{Det}_{\mathbb{Q}_{p}^{\text {ur }}[G]}(0) \stackrel{\epsilon_{\mathbb{Q}_{p}[G], \zeta}(V)}{\longrightarrow} \mathbb{Q}_{p}^{\mathrm{ur}}[G] \underset{\mathbb{Q}_{p}[G]}{\otimes}\left(\operatorname{Det}_{\mathbb{Q}_{p}[G]} R \Gamma\left(K, \mathbb{Q}_{p}(1-r)\right) \cdot \operatorname{Det}_{\mathbb{Q}_{p}[G]}(V)\right) \\
& \begin{array}{c}
\uparrow\left[\frac{1-p^{-r} \sigma^{-1}}{1-p^{r-1} \sigma}\right]_{F} \\
\operatorname{Det}_{\mathbb{Q}_{p}^{\text {ur }}[G]}(0) \stackrel{\theta^{\prime \prime}}{\longrightarrow} \mathbb{Q}_{p}^{\text {ur }}[G] \underset{\mathbb{Q}_{p}[G]}{\otimes}\left(\operatorname{Det}_{\mathbb{Q}_{p}[G]}^{-1} H^{1}\left(K, \mathbb{Q}_{p}(1-r)\right) \cdot \operatorname{Det}_{\mathbb{Q}_{p}[G]}\left(\mathbb{Q}_{p}[G]\right)\right)
\end{array}
\end{aligned}
$$

where

$$
\theta^{\prime \prime}=\left[(-1)^{r-1}(r-1) !\right] \cdot \epsilon\left(K / \mathbb{Q}_{p}, 1-r\right) \cdot \operatorname{Det}_{\overline{\mathbb{Q}}_{p}[G]}(\text { per }) \cdot \theta^{\prime}
$$

and $c$ involves passage to cohomology as well as our identification $V \cong \mathbb{Q}_{p}[G]$ chosen above. Now passage to cohomology is also the scalar extension of the isomorphism

$$
\operatorname{Det}_{\mathbb{Z}_{p}[G]}^{-1}\left(\mathbb{Z}_{p}[G] \cdot \beta\right) \cdot \operatorname{Det}_{\mathbb{Z}_{p}[G]}\left(C_{\beta}\right) \cong \operatorname{Det}_{\mathbb{Z}_{p}[G]} R \Gamma\left(K, \mathbb{Z}_{p}(1-r)\right)
$$

induced by the short exact sequence of perfect complexes of $\mathbb{Z}_{p}[G]$-modules

$$
0 \rightarrow R \Gamma\left(K, \mathbb{Z}_{p}(1-r)\right) \rightarrow C_{\beta} \rightarrow \mathbb{Z}_{p}[G] \cdot \beta \rightarrow 0
$$

combined with the acyclicity isomorphism

$$
\text { can : } \operatorname{Det}_{\mathbb{Q}_{p}[G]}(0) \cong \operatorname{Det}_{\mathbb{Q}_{p}[G]}\left(C_{\beta, \mathbb{Q}_{p}}\right) \text {. }
$$


Since the class of $C_{\beta}$ in $K_{0}\left(\mathbb{Z}_{p}[G]\right)$ vanishes, we can choose an isomorphism

$$
a: \operatorname{Det}_{\mathbb{Z}_{p}[G]}(0) \cong \operatorname{Det}_{\mathbb{Z}_{p}[G]}\left(C_{\beta}\right),
$$

which leads to another isomorphism

$$
c^{\prime}: \operatorname{Det}_{\mathbb{Z}_{p}[G]}^{-1}\left(\mathbb{Z}_{p}[G] \cdot \beta\right) \cong \operatorname{Det}_{\mathbb{Z}_{p}[G]} R \Gamma\left(K, \mathbb{Z}_{p}(1-r)\right)
$$

defined over $\mathbb{Z}_{p}[G]$. Setting

$$
\lambda:=\left(c_{\mathbb{Q}_{p}}^{\prime}\right)^{-1} c \in \operatorname{Aut}\left(\operatorname{Det}_{\mathbb{Q}_{p}[G]}^{-1} H^{1}\left(K, \mathbb{Q}_{p}(1-r)\right)\right)=K_{1}\left(\mathbb{Q}_{p}[G]\right),
$$

we obtain a commutative diagram

$$
\begin{aligned}
& \operatorname{Det}_{\mathbb{Q}_{p}^{\mathrm{ur}}[G]}(0) \stackrel{\epsilon_{\mathbb{Q}_{p}[G], \zeta}(V)}{\longrightarrow} \mathbb{Q}_{p}^{\mathrm{ur}}[G] \underset{\mathbb{Q}_{p}[G]}{\otimes}\left(\operatorname{Det}_{\mathbb{Q}_{p}[G]} R \Gamma\left(K, \mathbb{Q}_{p}(1-r)\right) \cdot \operatorname{Det}_{\mathbb{Q}_{p}[G]}(V)\right) \\
& \uparrow\left[\frac{1-p^{-r} \sigma^{-1}}{1-p^{r-1} \sigma}\right]_{F} \quad \uparrow c_{\mathbb{Q}_{p}}^{\prime} \\
& \operatorname{Det}_{\mathbb{Q}_{p}^{\text {ur }}[G]}(0) \stackrel{\theta^{\prime \prime \prime}}{\longrightarrow} \mathbb{Q}_{p}^{\mathrm{ur}}[G] \underset{\mathbb{Q}_{p}[G]}{\otimes}\left(\operatorname{Det}_{\mathbb{Q}_{p}[G]}^{-1} H^{1}\left(K, \mathbb{Q}_{p}(1-r)\right) \cdot \operatorname{Det}_{\mathbb{Q}_{p}[G]}\left(\mathbb{Q}_{p}[G]\right)\right)
\end{aligned}
$$

where

$$
\theta^{\prime \prime \prime}=\lambda \circ \theta^{\prime \prime}=\lambda \cdot\left[(-1)^{r-1}(r-1) !\right] \cdot \epsilon\left(K / \mathbb{Q}_{p}, 1-r\right) \cdot \operatorname{Det}_{\overline{\mathbb{Q}}_{p}[G]}(\text { per }) \cdot \theta^{\prime} .
$$

The local Tamagawa number conjecture claims that $\epsilon_{\mathbb{Q}_{p}[G], \zeta}(V)$ is induced by an isomorphism

$$
\operatorname{Det}_{\mathbb{Z}_{p}^{\text {ur }}[G]}(0) \stackrel{\epsilon_{\mathbb{Z}_{p}[G], \zeta}(T)}{\longrightarrow} \mathbb{Z}_{p}^{\mathrm{ur}}[G] \underset{\mathbb{Z}_{p}[G]}{\otimes}\left(\operatorname{Det}_{\mathbb{Z}_{p}[G]} R \Gamma\left(K, \mathbb{Z}_{p}(1-r)\right) \cdot \operatorname{Det}_{\mathbb{Z}_{p}[G]}(T)\right)
$$

and this will be the case if and only if

$$
\theta^{\mathrm{iv}}:=\theta^{\prime \prime \prime} \cdot\left[\frac{1-p^{r-1} \sigma}{1-p^{-r} \sigma^{-1}}\right]_{F}
$$

is induced by an isomorphism

$$
\operatorname{Det}_{\mathbb{Z}_{p}^{\text {ur }[G]}}(0) \stackrel{\theta_{\mathbb{Z}_{p}[G]}^{\text {iv }}}{\longrightarrow} \mathbb{Z}_{p}^{\text {ur }}[G] \underset{\mathbb{Z}_{p}[G]}{\otimes}\left(\operatorname{Det}_{\mathbb{Z}_{p}[G]}^{-1}\left(\mathbb{Z}_{p}[G] \cdot \beta\right) \cdot \operatorname{Det}_{\mathbb{Z}_{p}[G]}\left(\mathbb{Z}_{p}[G]\right)\right) .
$$

The isomorphism of $\overline{\mathbb{Q}}_{p}[G]$-modules

$\tau: H^{1}\left(K, \mathbb{Q}_{p}(1-r)\right) \otimes_{\mathbb{Q}_{p}} \overline{\mathbb{Q}}_{p} \stackrel{\exp ^{*} \otimes \overline{\mathbb{Q}}_{p}}{\longrightarrow} K \otimes_{\mathbb{Q}_{p}} \overline{\mathbb{Q}}_{p} \stackrel{\text { per }}{\longrightarrow} \overline{\mathbb{Q}}_{p}[G] \stackrel{\cdot \operatorname{per}\left(\exp ^{*}(\beta)\right)^{-1}}{\longrightarrow} \overline{\mathbb{Q}}_{p}[G]$ is clearly induced by an isomorphism of $\mathbb{Z}_{p}[G]$-modules

$$
\tau_{\mathbb{Z}_{p}[G]}: \mathbb{Z}_{p}[G] \cdot \beta \stackrel{\sim}{\rightarrow} \mathbb{Z}_{p}[G]
$$


and we have

$$
\begin{aligned}
& \theta^{\mathrm{iv}}=\left[\frac{1-p^{r-1} \sigma}{1-p^{-r} \sigma^{-1}}\right]_{F} \cdot \lambda \cdot\left[(-1)^{r-1}(r-1) !\right] \\
& \cdot \epsilon\left(K / \mathbb{Q}_{p}, 1-r\right) \cdot\left[\operatorname{per}\left(\exp ^{*}(\beta)\right)\right] \cdot \operatorname{Det}_{\overline{\mathbb{Q}}_{p}[G]}(\tau) .
\end{aligned}
$$

Hence $\theta^{\text {iv }}$ is induced by an isomorphism $\theta_{\mathbb{Z}_{p}[G]}^{\text {iv }}$ if and only if the class in $K_{1}\left(\mathbb{Q}_{p}^{\text {ur }}[G]\right)$ of

$$
\left[\frac{1-p^{r-1} \sigma}{1-p^{-r} \sigma^{-1}}\right]_{F} \cdot \lambda \circ\left[(-1)^{r-1}(r-1) !\right] \cdot \epsilon\left(K / \mathbb{Q}_{p}, 1-r\right) \cdot\left[\operatorname{per}\left(\exp ^{*}(\beta)\right)\right]
$$

lies in $K_{1}\left(\mathbb{Z}_{p}^{\text {ur }}[G]\right)$. Now note that $[(-1)] \in K_{1}(\mathbb{Z}) \subset K_{1}\left(\mathbb{Z}_{p}^{\text {ur }}[G]\right)$ and that $\lambda=$ $\left[C_{\beta}\right]^{-1}$, so we do indeed obtain identity (4). In order to see this last identity, note that we have

$$
\lambda^{-1}=a^{-1} \cdot \text { can }
$$

and that $a^{-1} \cdot$ can $\in K_{1}\left(\mathbb{Q}_{p}^{\mathrm{ur}}[G]\right)$ is a lift of $\left[C_{\beta}\right] \in K_{0}\left(\mathbb{Z}_{p}^{\mathrm{ur}}[G], \mathbb{Q}_{p}\right)$ according to the conventions of [Fukaya and Kato 2006, 1.3.8, Theorem 1.3.15(ii)].

2.1. Description of $\boldsymbol{K}_{\mathbf{1}}$. For any finite group $G$ we have the Wedderburn decomposition

$$
\overline{\mathbb{Q}}_{p}[G] \cong \prod_{\chi \in \widehat{G}} M_{d_{\chi}}\left(\overline{\mathbb{Q}}_{p}\right)
$$

where $\widehat{G}$ is the set of irreducible $\overline{\mathbb{Q}}_{p}$-valued characters of $G$ and $d_{\chi}=\chi(1)$ is the degree of $\chi$. Hence there is a corresponding decomposition

$$
K_{1}\left(\overline{\mathbb{Q}}_{p}[G]\right) \cong \prod_{\chi \in \widehat{G}} K_{1}\left(M_{d_{\chi}}\left(\overline{\mathbb{Q}}_{p}\right)\right) \cong \prod_{\chi \in \widehat{G}} \overline{\mathbb{Q}}_{p}^{\times},
$$

which allows one to think of $K_{1}\left(\overline{\mathbb{Q}}_{p}[G]\right)$ as a collection of nonzero $p$-adic numbers indexed by $\widehat{G}$. Note here that for any ring $R$ one has $K_{1}\left(M_{d}(R)\right)=K_{1}(R)$ and for a commutative semilocal ring $R$ one has $K_{1}(R)=R^{\times}$.

If $p \nmid|G|$ then all characters $\chi \in \widehat{G}$ take values in $\mathbb{Z}_{p}^{\mathrm{ur}}$, the Wedderburn decomposition is already defined over $\mathbb{Z}_{p}^{\text {ur }}$ and so is the decomposition of $K_{1}$. One has

and

$$
K_{1}\left(\mathbb{Z}_{p}^{\mathrm{ur}}[G]\right) \cong \prod_{\chi \in \widehat{G}} K_{1}\left(M_{d_{\chi}}\left(\mathbb{Z}_{p}^{\mathrm{ur}}\right)\right) \cong \prod_{\chi \in \widehat{G}} \mathbb{Z}_{p}^{\mathrm{ur}, \times}
$$

$$
K_{1}\left(\mathbb{Q}_{p}^{\mathrm{ur}}[G]\right) / \operatorname{im}\left(K_{1}\left(\mathbb{Z}_{p}^{\mathrm{ur}}[G]\right)\right) \cong \prod_{\chi \in \widehat{G}} \mathbb{Q}_{p}^{\mathrm{ur}, \times} / \mathbb{Z}_{p}^{\mathrm{ur}, \times} \cong \prod_{\chi \in \widehat{G}} p^{\mathbb{Z}},
$$

which allows one to think of elements in $K_{1}\left(\mathbb{Q}_{p}^{\text {ur }}[G]\right) / \operatorname{im}\left(K_{1}\left(\mathbb{Z}_{p}^{\mathrm{ur}}[G]\right)\right)$ as a collection of integers ( $p$-adic valuations) indexed by $\widehat{G}$. 
2.2. Definition of the $\epsilon$-factor. If $L$ is a local field, $E$ an algebraically closed field of characteristic 0 with the discrete topology, $\mu_{L}$ a Haar measure on the additive group of $L$ with values in $E, \psi_{L}: L \rightarrow E^{\times}$a continuous character, the theory of Langlands-Deligne [Deligne 1973] associates to each continuous representation $r$ of the Weil group $W_{L}$ over $E$ an $\epsilon$-factor $\epsilon\left(r, \psi_{L}, \mu_{L}\right) \in E^{\times}$.

We shall take $E=\overline{\mathbb{Q}}_{p}$ and always fix $\mu_{L}$ and $\psi_{L}$ so that $\mu_{L}\left(\mathcal{O}_{L}\right)=1$ and $\psi_{L}=\psi_{\mathbb{Q}_{p}} \circ \operatorname{Tr}_{L / \mathbb{Q}_{p}}$ where $\psi_{\mathbb{Q}_{p}}\left(p^{-n}\right)=\zeta_{p^{n}}$ for our fixed $\zeta=\left(\zeta_{p^{n}}\right)_{n} \in \Gamma\left(\overline{\mathbb{Q}}_{p}, \mathbb{Z}_{p}(1)\right)$. Setting

$$
\epsilon(r):=\epsilon\left(r, \psi_{L}, \mu_{L}\right) \in E^{\times}
$$

and leaving the dependence on $\zeta$ implicit, we have the following properties (see also [Benois and Berger 2008] for a review, [Fukaya and Kato 2006] only reviews the case $L=\mathbb{Q}_{p}$ ). Let $\pi$ be a uniformizer of $\mathcal{O}_{L}, \delta_{L}$ the exponent of the different of $L / \mathbb{Q}_{p}$ and $q=\left|\mathcal{O}_{L} / \pi\right|$.

(a) If $r: W_{L} \rightarrow E^{\times}$is a homomorphism, set

$$
r_{\sharp}: L^{\times} \stackrel{\mathrm{rec}}{\rightarrow} W_{L}^{\mathrm{ab}} \stackrel{r}{\rightarrow} E^{\times}
$$

where rec is normalized as in [Deligne 1973, (2.3)] and sends a uniformizer to a geometric Frobenius automorphism in $W_{L}^{\text {ab }}$. Then we have

$$
\epsilon(r)= \begin{cases}q^{\delta_{L}} & \text { if } c=0, \\ q^{\delta_{L}} r_{\sharp}\left(\pi^{c+\delta_{L}}\right) \tau\left(r_{\sharp}, \psi_{\pi}\right) & \text { if } c>0,\end{cases}
$$

where $c \in \mathbb{Z}$ is the conductor of $r$ and

$$
\tau\left(r_{\sharp}, \psi_{\pi}\right)=\sum_{u \in\left(\mathcal{O}_{L} / \pi^{c}\right)^{\times}} r_{\sharp}^{-1}(u) \psi_{\pi}(u)
$$

is the Gauss sum associated to the restriction of $r_{\sharp}$ to $\left(\mathcal{O}_{L} /\left(\pi^{c}\right)\right)^{\times}$and the additive character

$$
u \mapsto \psi_{\pi}(u):=\psi_{K}\left(\pi^{-\delta_{L}-c} u\right)
$$

of $\mathcal{O}_{L} /\left(\pi^{c}\right)$.

(b) If $L / K$ is unramified then $\epsilon(r)=\epsilon\left(\operatorname{Ind}_{W_{L}}^{W_{K}} r\right)$ for any representation $r$ of $W_{L}$.

(c) If $r(\alpha)$ is the twist of $r$ with the unramified character with Frob $L_{L}$-eigenvalue $\alpha \in E^{\times}$, and $c(r) \in \mathbb{Z}$ is the conductor of $r$, then

$$
\epsilon(r(\alpha))=\alpha^{-c(r)-\operatorname{dim}_{E}(r) \delta_{L}} \epsilon(r) .
$$

Here Frob $_{L}$ denotes the usual (arithmetic) Frobenius automorphism.

For a potentially semistable representation $V$ of $G_{\mathbb{Q}_{p}}$ one first forms $D_{\text {pst }}(V)$, a finite dimensional $\widehat{\mathbb{Q}}_{p}^{\text {ur }}$-vector space of dimension $\operatorname{dim}_{\mathbb{Q}_{p}} V$ with an action of $G_{\mathbb{Q}_{p}}$, semilinear with respect to the natural action of $G_{\mathbb{Q}_{p}}$ on $\widehat{\mathbb{Q}}_{p}^{u r}$ and discrete on the inertia subgroup. Moreover, $D_{\mathrm{pst}}(V)$ has a Frob-semilinear automorphism $\varphi$. The 
associated linear representation $r_{V}$ of $W_{\mathbb{Q}_{p}}$ over $E=\widehat{\mathbb{Q}}_{p}^{\text {ur }}$ is the space $D_{\mathrm{pst}}(V)$ with action

$$
r_{V}(w)(d)=\iota(w) \varphi^{-v(w)}(d),
$$

where $\iota: W_{\mathbb{Q}_{p}} \rightarrow G_{\mathbb{Q}_{p}}$ is the inclusion and $v(w) \in \mathbb{Z}$ is such that $\operatorname{Frob}^{v(w)}$ is the image of $w$ in $G_{\mathbb{F}_{p}}$.

From now on we are interested in $V=\left(\operatorname{Ind}_{G_{K}}^{G_{Q_{p}}} \mathbb{Q}_{p}\right)(1-r)$. Here one has

$$
D_{\mathrm{pst}}(V)=\left(\operatorname{Ind}_{G_{K}}^{G_{\mathbb{Q}_{p}}} \widehat{\mathbb{Q}}_{p}^{\mathrm{ur}}\right) \cdot t^{r-1}, \quad r_{V}=\left(\operatorname{Ind}_{W_{K}}^{W_{\mathbb{Q}_{p}}} \widehat{\mathbb{Q}}_{p}^{\mathrm{ur}}\right)\left(p^{1-r}\right),
$$

and we notice that $r_{V}$ is the scalar extension from $\mathbb{Q}_{p}^{\mathrm{ur}}$ to $\widehat{\mathbb{Q}}_{p}^{\mathrm{ur}}$ of the representation $\left(\operatorname{Ind}_{W_{K}}^{W_{\mathbb{Q}_{p}}} \mathbb{Q}_{p}^{\text {ur }}\right)\left(p^{1-r}\right)$. So completion of $\mathbb{Q}_{p}^{\text {ur }}$ is not needed in this example. Associated to $r_{V} \otimes_{\mathbb{Q}_{p}^{\text {ur }}} \mathbb{Q}_{p}$ is an $\epsilon$-factor in $\epsilon\left(r_{V}\right) \in \overline{\mathbb{Q}}_{p}^{\times}=K_{1}\left(\overline{\mathbb{Q}}_{p}\right)$. However, as explained above before (3), $r_{V}$ carries a left action of $\mathbb{Q}_{p}^{\text {ur }}[G]$ commuting with the left $W_{\mathbb{Q}_{p}}$-action, so we will actually be able to associate to $r_{V} \otimes_{\mathbb{Q}_{p}^{\text {ur }}} \overline{\mathbb{Q}}_{p}$ a refined $\epsilon$-factor

$$
\epsilon\left(K / \mathbb{Q}_{p}, 1-r\right) \in K_{1}\left(\overline{\mathbb{Q}}_{p}[G]\right) .
$$

For each $\chi \in \widehat{G}$ define a representation $r_{\chi}$ of $W_{\mathbb{Q}_{p}}$ over $E=\overline{\mathbb{Q}}_{p}$ by

$$
W_{\mathbb{Q}_{p}} \stackrel{\iota}{\rightarrow} G_{\mathbb{Q}_{p}} \stackrel{\psi}{\rightarrow} G \stackrel{\rho_{\chi}}{\rightarrow} \mathrm{GL}_{d_{\chi}}(E),
$$

where $\rho_{\chi}: G \rightarrow \mathrm{GL}_{d_{\chi}}(E)$ is a homomorphism realizing $\chi$. Let $E^{d_{\chi}}$ be the space of row vectors on which $G$ acts on the right via $\rho_{\chi}$ and define another representation of $W_{\mathbb{Q}_{p}}$ over $E=\overline{\mathbb{Q}}_{p}$

$$
r_{V, \chi}=E^{d_{\chi}} \otimes_{\mathbb{Q}_{p}^{\text {ur }}[G]} r_{V}=E^{d_{\chi}} \otimes_{\mathbb{Q}_{p}^{\text {ur }}[G]}\left(\operatorname{Ind}_{W_{K}}^{W_{\mathbb{Q}_{p}}} \mathbb{Q}_{p}^{\mathrm{ur}}\right)\left(p^{1-r}\right) \cong E^{d_{\chi}} .
$$

By (3), the left $W_{\mathbb{Q}_{p}}$-action on this last space is given by the contragredient ${ }^{t} \rho_{\chi}(\psi(g))^{-1}$ of $r_{\chi}$, twisted by the unramified character with eigenvalue $p^{1-r}$. So we have

$$
r_{V, \chi} \cong r_{\bar{\chi}}\left(p^{1-r}\right),
$$

where $\bar{\chi}$ is the contragredient character of $\chi$. We view the collection

$$
\epsilon(K / \mathbb{Q}, 1-r):=\left(\epsilon\left(r_{V, \chi}\right)\right)_{\chi \in \widehat{G}}=\left(\epsilon\left(r_{\bar{\chi}}\right) p^{(r-1) c\left(r_{\bar{\chi}}\right)}\right)_{\chi \in \widehat{G}}
$$

as an element of $K_{1}\left(\overline{\mathbb{Q}}_{p}[G]\right)$ in the description (5).

\section{The conjecture in the case $p \nmid|G|$}

In this section and for most of the rest of the paper we assume that $p$ does not divide $|G|=\left[K: \mathbb{Q}_{p}\right]$. In particular $K / \mathbb{Q}_{p}$ is tamely ramified with maximal unramified subfield $F$. Although our methods probably extend to an arbitrary tamely ramified extension $K / \mathbb{Q}_{p}$ (i.e., where $p$ is allowed to divide $\left[F: \mathbb{Q}_{p}\right]$ ) this would add an 
extra layer of notational complexity which we have preferred to avoid. The group $G=\operatorname{Gal}\left(K / \mathbb{Q}_{p}\right)$ is an extension of two cyclic groups

$$
\begin{aligned}
\Sigma & :=\operatorname{Gal}\left(F / \mathbb{Q}_{p}\right) \cong \mathbb{Z} / f \mathbb{Z}, \\
\Delta & :=\operatorname{Gal}(K / F) \cong \mathbb{Z} / e \mathbb{Z},
\end{aligned}
$$

where the action of $\sigma \in \Sigma$ on $\Delta$ is given by $\delta \mapsto \delta^{p}$ and we have $e \mid p^{f}-1$. By Kummer theory $K=F\left(\sqrt[e]{p_{0}}\right)$, where $p_{0} \in\left(F^{\times} /\left(F^{\times}\right)^{e}\right)^{\Sigma}$ has order $e$. We can and will assume that $p_{0}$ has $p$-adic valuation one, and in fact that $p_{0}=\lambda \cdot p$ with $\lambda \in \mu_{F}$. Writing $p_{0}=\lambda^{\prime} \cdot p_{0}^{\prime}$ with $p_{0}^{\prime} \in \mathbb{Q}_{p}$ we see that $K$ is contained in $F^{\prime}\left(\sqrt[e]{p_{0}^{\prime}}\right)$, where $F^{\prime}:=F\left(\sqrt[e]{\lambda^{\prime}}\right)$ is unramified over $\mathbb{Q}_{p}$ and $p_{0}^{\prime}$ is any choice of element in $\mu_{\mathbb{Q}_{p}} \cdot p=\mu_{p-1} \cdot p$. Since for the purpose of proving the local Tamagawa number conjecture we can always enlarge $K$, we may and will assume that

$$
K=F\left(\sqrt[e]{p_{0}}\right), \quad p_{0} \in \mu_{p-1} \cdot p \subseteq \mathbb{Q}_{p} .
$$

We then have

$$
G=\operatorname{Gal}\left(K / \mathbb{Q}_{p}\right) \cong \Sigma \ltimes \Delta
$$

since $\operatorname{Gal}\left(K / \mathbb{Q}_{p}\left(\sqrt[e]{p_{0}}\right)\right)$ is a complement of $\Delta$. If $(e, p-1)=1$, then the fields $K=F\left(\sqrt[e]{p_{0}}\right)$ for $p_{0} \in \mu_{p-1} \cdot p$ are all isomorphic; in fact any Galois extension $K / \mathbb{Q}_{p}$ with invariants $e$ and $f$ is then isomorphic to the field $F(\sqrt[e]{p})$.

The choice of $p_{0}$ (in fact just the valuation of $p_{0}$ ) determines a character

$$
\eta_{0}: \Delta \stackrel{\sim}{\longrightarrow} \mu_{e} \subset F^{\times} \subset \mathbb{Q}_{p}^{\mathrm{ur}, \times} \subset \overline{\mathbb{Q}}_{p}^{\times}
$$

by the usual formula $\delta\left(\sqrt[e]{p_{0}}\right)=\eta_{0}(\delta) \cdot \sqrt[e]{p_{0}}$. Let

$$
\eta: \Delta \rightarrow F^{\times}
$$

be any character of $\Delta$ and

$$
\Sigma_{\eta}:=\left\{g \in \Sigma \mid \eta\left(g \delta g^{-1}\right)=\eta(\delta) \text { for all } \delta \in \Delta\right\}
$$

the stabilizer of $\eta$. Then for any character $\eta^{\prime}: \Sigma_{\eta} \rightarrow \mathbb{Q}_{p}^{\mathrm{ur}, \times}$ we obtain a character

$$
\eta^{\prime} \eta: G_{\eta}:=\Sigma_{\eta} \ltimes \Delta \rightarrow \mathbb{Q}_{p}^{\mathrm{ur}, \times}
$$

and an induced character

$$
\chi:=\operatorname{Ind}_{G_{\eta}}^{G}\left(\eta^{\prime} \eta\right)
$$

of $G$. By [Lang 2002, Exercise XVIII.7], all irreducible characters of $G$ are obtained by this construction, and in fact each $\chi \in \widehat{G}$ is parametrized by a unique pair $\left([\eta], \eta^{\prime}\right)$ where $[\eta]$ denotes the $\Sigma$-orbit of $\eta$. The degree of $\chi$ is given by

$$
d_{\chi}=\chi(1)=f_{\eta}:=\left[\Sigma: \Sigma_{\eta}\right]=\left[F_{\eta}: \mathbb{Q}_{p}\right],
$$

where $F_{\eta} \subseteq F$ is the fixed field of $\Sigma_{\eta}$. 
We have

$$
r_{\chi}=\operatorname{Ind}_{W_{F_{\eta}}}^{W_{\mathbb{Q}_{p}}}\left(r_{\eta^{\prime} \eta}\right),
$$

where $r_{\chi}$ and $r_{\eta^{\prime} \eta}$ are the representations of $W_{\mathbb{Q}_{p}}$ and $W_{F_{\eta}}$, respectively, defined as in (8). By [Serre 1979, Chapter VI, Corollary to Propoposition 4] we have

$$
c\left(r_{\chi}\right)=f_{\eta} c\left(r_{\eta}\right)= \begin{cases}0, & \eta=1, \\ f_{\eta}, & \eta \neq 1 .\end{cases}
$$

Using (b), (c) and (a) of Section 2.2 we have

$$
\begin{aligned}
\epsilon\left(r_{\chi}\right) & =\epsilon\left(r_{\eta^{\prime} \eta}\right) \\
& = \begin{cases}1, & \eta=1, \\
\epsilon\left(r_{\eta}\right) r_{\eta^{\prime}}\left(\operatorname{Frob}_{F_{\eta}}\right)^{-c\left(r_{\eta}\right)}=\eta(\operatorname{rec}(p)) \tau\left(r_{\eta, \sharp}, \psi_{p}\right) \eta^{\prime}\left(\sigma^{f_{\eta}}\right)^{-1}, & \eta \neq 1 .\end{cases}
\end{aligned}
$$

3.1. Gauss sums. If $k_{\eta}$ denotes the residue field of $F_{\eta}$, we have a canonical character

$$
\omega: k_{\eta}^{\times} \stackrel{\sim}{\longleftarrow} \mu_{p^{f_{\eta}-1}} \subseteq F_{\eta}^{\times} \subseteq K^{\times} \subseteq \overline{\mathbb{Q}}_{p}^{\times},
$$

where the first arrow is reduction mod $p$. On the other hand we have our character

$$
r_{\eta, \sharp}: F_{\eta}^{\times} \stackrel{\mathrm{rec}}{\rightarrow} W_{F_{\eta}}^{\mathrm{ab}} \stackrel{\iota}{\rightarrow} G_{F_{\eta}}^{\mathrm{ab}} \stackrel{\psi}{\rightarrow} G_{\eta}^{\mathrm{ab}} \stackrel{\eta}{\rightarrow} \overline{\mathbb{Q}}_{p}^{\times}
$$

of order dividing $e$. So there exists a unique $m_{\eta} \in \mathbb{Z} / e \mathbb{Z}$ such that

$$
\left.r_{\eta, \sharp}\right|_{\mu^{f_{\eta}}-1}=\omega^{m_{\eta}\left(p^{f_{\eta}}-1\right) / e}
$$

and formula (7) gives

$$
\tau\left(r_{\eta, \sharp}, \psi_{p}\right)=\tau\left(\omega^{-m_{\eta}\left(p^{f_{\eta}}-1\right) / e}\right),
$$

where

$$
\tau\left(\omega^{-i}\right):=\sum_{a \in k_{\eta}^{\times}} \omega(a)^{-i} \zeta_{p}^{\operatorname{Tr}_{k_{\eta} / \mathbb{F} p}(a)}
$$

is a Gauss sum associated to the finite field $k_{\eta}$. The $p$-adic valuation of these sums is known:

Lemma 3 [Washington 1997, Proposition 6.13 and Lemma 6.14]. For $0 \leq i \leq p^{f_{\eta}}-1$, let $i=i_{0}+p i_{1}+p^{2} i_{2}+\cdots+i_{f_{\eta}-1} p^{f_{\eta}-1}$ be the $p$-adic expansion with digits $0 \leq i_{j} \leq p-1$. Then

$$
v_{p}\left(\tau\left(\omega^{-i}\right)\right)=\frac{i_{0}+i_{1}+\cdots+i_{f_{\eta}-1}}{p-1}=\sum_{j=0}^{f_{\eta}-1}\left\langle\frac{i p^{j}}{p^{f_{\eta}}-1}\right\rangle,
$$

where $v_{p}: \overline{\mathbb{Q}}_{p}^{\times} \rightarrow \mathbb{Q}$ is the $p$-adic valuation on $\overline{\mathbb{Q}}_{p}$ normalized by $v_{p}(p)=1$ and $0 \leq\langle x\rangle<1$ is the fractional part of the real number $x$. 
Corollary 4. For all $\eta \in \hat{\Delta}$ we have

$$
v_{p}\left(\tau\left(r_{\eta, \sharp}, \psi_{p}\right)\right)=\sum_{j=0}^{f_{\eta}-1}\left\langle\frac{m_{\eta} p^{j}}{e}\right\rangle .
$$

After this interlude on Gauss sums we now prove a statement about periods of certain specific elements in $K$ which will eliminate any further reference to $\epsilon$-factors in the proof of Equation (4).

Proposition 5. Let $K / \mathbb{Q}_{p}$ be Galois with group $G$ of order prime to $p$. Then any fractional $\mathcal{O}_{K}$-ideal is a free $\mathbb{Z}_{p}[G]$-module of rank one and

$$
\left(\epsilon\left(r_{\bar{\chi}}\right)\right)_{\chi \in \widehat{G}} \cdot[\operatorname{per}(b)] \in \operatorname{im}\left(K_{1}\left(\mathbb{Z}_{p}^{\mathrm{ur}}[G]\right)\right)
$$

for any $\mathbb{Z}_{p}[G]$-basis $b$ of the inverse different $\left(\sqrt[e]{p_{0}}\right)^{-\delta_{K}} \mathcal{O}_{K}=\left(\sqrt[e]{p_{0}}\right)^{-(e-1)} \mathcal{O}_{K}$.

Proof. This is a classical result in Galois module theory which can be found in [Fröhlich 1976] but rather than trying to match our notation to that paper we go through the main computations again. In this proof $\sigma$ will temporarily denote a generic element of $\Sigma$ rather than the Frobenius.

The image of $[\operatorname{per}(b)]$ in the $\chi$-component of the decomposition (5) is the $\left(d_{\chi} \times d_{\chi}\right)$-determinant

$$
[\operatorname{per}(b)]_{\chi}:=\operatorname{det} \rho_{\chi}\left(\sum_{g \in G} g(b) \cdot g^{-1}\right)=\operatorname{det} \sum_{g \in G} g(b) \rho_{\chi}(g)^{-1} \in \overline{\mathbb{Q}}_{p}^{\times} .
$$

This character function is traditionally called a resolvent. With notation as above, $\left(\sqrt[e]{p_{0}}\right)^{-(e-1)} \mathcal{O}_{K}$ is a free $\mathbb{Z}_{p}\left[G_{\eta}\right]$-module with basis $\sigma(b)$, where $\sigma \in G_{\eta} \backslash G \cong \Sigma_{\eta} \backslash \Sigma$ runs through a set of right coset representatives. The image of this basis under the period map is

$$
\operatorname{per}(\sigma(b))=\sum_{g \in G} g \sigma(b) \cdot g^{-1}=\sum_{\tau \in \Sigma_{\eta} \backslash \Sigma}\left(\sum_{g \in G_{\eta}} \tau^{-1} g \sigma(b) \cdot g^{-1}\right) \tau
$$

and if $\chi=\operatorname{Ind}_{G_{\eta}}^{G}\left(\chi^{\prime}\right)$ is an induced character we have by [Fröhlich 1976, (5.15)]

$$
\rho_{\chi}\left(\sum_{g \in G} g(b) \cdot g^{-1}\right)=\left(\sum_{g \in G_{\eta}} \tau^{-1} g \sigma(b) \cdot \rho_{\chi^{\prime}}(g)^{-1}\right)_{\sigma, \tau} .
$$

In our case of interest $\chi^{\prime}=\eta^{\prime} \eta$ is a one-dimensional character. Write

$$
b=\xi \cdot x,
$$


where $x$ is an $\mathcal{O}_{F}[\Delta]$-basis of $\left(\sqrt[e]{p}_{0}\right)^{-(e-1)} \mathcal{O}_{K}$ fixed by $\Sigma$ and $\xi$ a $\mathbb{Z}_{p}[\Sigma]$-basis of $\mathcal{O}_{F}$. Then writing $g=\delta \sigma^{\prime}$ with $\delta \in \Delta$ and $\sigma^{\prime} \in \Sigma_{\eta}$ this matrix becomes

$$
\left(\sum_{\sigma^{\prime} \in \Sigma_{\eta}} \tau^{-1} \sigma^{\prime} \sigma(\xi) \eta^{\prime}\left(\sigma^{\prime}\right)^{-1} \sum_{\delta \in \Delta} \tau^{-1} \delta(x) \cdot \eta(\delta)^{-1}\right)_{\sigma, \tau}
$$

and its determinant is

$$
\operatorname{det}\left(\sum_{\sigma^{\prime} \in \Sigma_{\eta}} \tau^{-1} \sigma^{\prime} \sigma(\xi) \eta^{\prime}\left(\sigma^{\prime}\right)^{-1}\right)_{\sigma, \tau} \cdot \prod_{\tau \in \Sigma_{\eta} \backslash \Sigma} \sum_{\delta \in \Delta} \tau^{-1} \delta \tau(x) \cdot \eta(\delta)^{-1} .
$$

The first determinant is a group determinant [Washington 1997, Lemma 5.26] for the group $\Sigma_{\eta} \backslash \Sigma$ and equals

$$
\xi_{\eta^{\prime}}:=\prod_{\kappa \in\left(\Sigma_{\eta} \backslash \Sigma\right)^{\wedge}} \sum_{\sigma \in \Sigma_{\eta} \backslash \Sigma}\left(\sum_{\sigma^{\prime} \in \Sigma_{\eta}} \sigma^{\prime} \sigma(\xi) \eta^{\prime}\left(\sigma^{\prime}\right)^{-1}\right) \kappa(\sigma)^{-1}=\prod_{\kappa} \sum_{\sigma \in \Sigma} \sigma(\xi) \kappa(\sigma)^{-1},
$$

where this last product is over all characters $\kappa$ of $\Sigma$ restricting to $\eta^{\prime}$ on $\Sigma_{\eta}$. The sum $\sum_{\sigma \in \Sigma} \sigma(\xi) \kappa(\sigma)^{-1}$ clearly lies in $\mathbb{Z}_{p}^{\text {ur, } \times}$ since its reduction modulo $p$ is the projection of the $\overline{\mathbb{F}}_{p}[\Sigma]$-basis $\bar{\xi}$ of $\mathcal{O}_{F} /(p) \otimes_{\mathbb{F}_{p}} \overline{\mathbb{F}}_{p}$ into the $\bar{\kappa}$-eigenspace (up to the unit $|\Sigma|=f$ ), hence nonzero. So we find

$$
\xi_{\eta^{\prime}} \in \mathbb{Z}_{p}^{\mathrm{ur}, \times}
$$

We now analyze the second factor

$$
x_{\eta}:=\prod_{\tau \in \Sigma_{\eta} \backslash \Sigma} \sum_{\delta \in \Delta} \tau^{-1} \delta \tau(x) \cdot \eta(\delta)^{-1}
$$

which is the product over the projections of $x$ into the $\eta^{p^{i}}$-eigenspaces for $i=$ $0, \ldots, f_{\eta}-1$ (up to the unit $|\Delta|=e$ ). For $0 \leq j<e$ the $\eta_{0}^{-j}$-eigenspace of the inverse different is generated over $\mathcal{O}_{F}$ by $\left(\sqrt[e]{p_{0}}\right)^{-j}$ and since $x$ was a $\mathcal{O}_{F}[\Delta]$-basis of the inverse different its projection lies in $\mathcal{O}_{F}^{\times} \cdot\left(\sqrt[e]{p_{0}}\right)^{-j}$. So by Lemma 6 below we have

$$
x_{\eta} \in \mathcal{O}_{F}^{\times} \cdot \prod_{i=0}^{f_{\eta}-1}\left(\sqrt[e]{p_{0}}\right)^{-e\left\langle p^{i}\left(-m_{\eta}\right) / e\right\rangle} \subset K
$$

and hence

$$
v_{p}\left(x_{\eta}\right)=-\sum_{i=0}^{f_{\eta}-1}\left\langle\frac{-m_{\eta} p^{i}}{e}\right\rangle=-v_{p}\left(\tau\left(r_{\bar{\eta}, \sharp}, \psi_{p}\right)\right),
$$

using Corollary 4 and the fact that $\bar{\eta}=\eta_{0}^{-m_{\eta}}$. One checks that $\tau\left(r_{\bar{\eta}, \sharp}, \psi_{p}\right) \in \mathbb{Q}_{p}^{\mathrm{ur}}\left(\zeta_{p}\right)$ is an eigenvector for the character

$$
\varrho=\eta_{0}^{-m_{\eta}\left(p^{f_{\eta}}-1\right) /(p-1)}
$$


of the group $\operatorname{Gal}\left(\mathbb{Q}_{p}^{\mathrm{ur}}\left(\zeta_{p}\right) \cap K^{\mathrm{ur}} / \mathbb{Q}_{p}^{\mathrm{ur}}\right)$. Also, since $x_{\eta}$ is an eigenvector for $\varrho^{-1}$, Equation (15) implies

$$
\tau\left(r_{\bar{\eta}, \sharp}, \psi_{p}\right) \cdot x_{\eta} \in \mathbb{Z}_{p}^{\mathrm{ur}, \times} .
$$

Combining this with (14) and (12) we find

$$
\epsilon\left(r_{\bar{\chi}}\right) \cdot[\operatorname{per}(b)]_{\chi}=\bar{\eta}(\operatorname{rec}(p)) \tau\left(r_{\bar{\eta}, \sharp}, \psi_{p}\right) \bar{\eta}^{\prime}\left(\sigma^{f_{\eta}}\right) \cdot x_{\eta} \cdot \xi_{\eta^{\prime}} \in \mathbb{Z}_{p}^{\mathrm{ur}, \times}
$$

and hence

$$
\left(\epsilon\left(r_{\bar{\chi}}\right)\right)_{\chi \in \widehat{G}} \cdot[\operatorname{per}(b)] \in \operatorname{im}\left(K_{1}\left(\mathbb{Z}_{p}^{\mathrm{ur}}[G]\right)\right) .
$$

Lemma 6. We have $\eta=\eta_{0}^{m_{\eta}}$, where $\eta_{0}$ is the character (10) associated to the element $p_{0}$ of valuation 1 and $m_{\eta}$ was defined in (13).

Proof. It suffices to show that the composite map

$$
\omega^{\prime}: \mu_{p^{f_{\eta}-1}} \subset F^{\times} \stackrel{\mathrm{rec}}{\longrightarrow} G_{F}^{\mathrm{ab}} \rightarrow \operatorname{Gal}(K / F) \stackrel{\eta_{0}^{m_{\eta}}}{\longrightarrow} \mu_{e}
$$

agrees with the $\left(m_{\eta}\left(p^{f_{\eta}}-1\right) / e\right)$-th power map. By definition [Neukirch 1999, Theorem V.3.1] of the tame local Hilbert symbol and the fact that our map rec is the inverse of that used in [Neukirch 1999], we have

$$
\omega^{\prime}(\zeta)=\left(\frac{\zeta^{-1}, p_{0}^{m_{\eta}}}{F}\right)
$$

which by [Neukirch 1999, Theorem V.3.4] equals

$$
\left(\frac{\zeta^{-1}, p_{0}^{m_{\eta}}}{F}\right)=\left((-1)^{\alpha \beta} \frac{p_{0}^{\beta}}{\zeta^{-\alpha}}\right)^{\left(p^{f_{\eta}}-1\right) / e}=\zeta^{m_{\eta}\left(p^{f_{\eta}}-1\right) / e},
$$

where $\alpha=v_{p}\left(p_{0}^{m_{\eta}}\right)=m_{\eta}$ and $\beta=v_{p}\left(\zeta^{-1}\right)=0$.

Denote by $\gamma$ a topological generator of

$$
\Gamma:=\operatorname{Gal}\left(\mathbb{Q}_{p}\left(\zeta_{p^{\infty}}\right) / \mathbb{Q}_{p}\right)
$$

and by

$$
\chi^{\text {cyclo }}: \operatorname{Gal}\left(\mathbb{Q}_{p}\left(\zeta_{p^{\infty}}\right) / \mathbb{Q}_{p}\right) \cong \mathbb{Z}_{p}^{\times}
$$

the cyclotomic character. As in the proof of Proposition 5 choose $b$ such that

$$
\mathbb{Z}_{p}[G] \cdot b=\left(\sqrt[e]{p_{0}}\right)^{-(e-1)} \mathcal{O}_{K} .
$$

Denote by $e_{1}=\frac{1}{|\Sigma|} \sum_{g \in \Sigma} g \in \mathbb{Z}_{p}[\Sigma]$ the idempotent for the trivial character of $\Sigma$.

Proposition 7. If $p \nmid|G|$ then one can choose $\beta \in H^{1}\left(K, \mathbb{Z}_{p}(1-r)\right)$ such that

$$
H^{1}\left(K, \mathbb{Z}_{p}(1-r)\right)=H^{1}\left(K, \mathbb{Z}_{p}(1-r)\right)_{\text {tor }} \oplus \mathbb{Z}_{p}[G] \cdot \beta
$$


and the local Tamagawa number conjecture (4) is equivalent to the identity

$[(r-1) !] \cdot\left(p^{(r-1) c(\chi)}\right)_{\chi \in \widehat{G}} \cdot[\operatorname{per}(b)]^{-1} \cdot\left[\operatorname{per}\left(\exp ^{*}(\beta)\right)\right] \cdot\left[C_{\beta}\right]^{-1} \cdot\left[\frac{1-p^{r-1} \sigma}{1-p^{-r} \sigma^{-1}}\right]_{F}=1$

in the group $K_{1}\left(\mathbb{Q}_{p}^{\mathrm{ur}}[G]\right) / \operatorname{im} K_{1}\left(\mathbb{Z}_{p}^{\mathrm{ur}}[G]\right)$. The projection of this identity into the group $K_{1}\left(\mathbb{Q}_{p}^{\mathrm{ur}}[\Sigma]\right) / \operatorname{im} K_{1}\left(\mathbb{Z}_{p}^{\mathrm{ur}}[\Sigma]\right)$ is

$[(r-1) !] \cdot\left[\operatorname{per}\left(\exp ^{*}(\beta)\right)\right]_{F} \cdot\left[\frac{\chi^{\mathrm{cyclo}}(\gamma)^{r}-1}{\chi^{\mathrm{cyclo}}(\gamma)^{r-1}-1} e_{1}+1-e_{1}\right] \cdot\left[\frac{1-p^{r-1} \sigma}{1-p^{-r} \sigma^{-1}}\right]_{F}=1$

and in the components of $K_{1}\left(\mathbb{Q}_{p}^{\mathrm{ur}}[G]\right) / \operatorname{im} K_{1}\left(\mathbb{Z}_{p}^{\mathrm{ur}}[G]\right)$ indexed by $\chi=\left([\eta], \eta^{\prime}\right)$ with

$$
\left.\eta\right|_{\mathrm{Gal}\left(K / K \cap F\left(\zeta_{p}\right)\right)} \neq 1
$$

this identity is equivalent to

$$
((r-1) !)^{f_{\eta}} \cdot p^{(r-1) f_{\eta}} \cdot[\operatorname{per}(b)]_{\chi}^{-1} \cdot\left[\operatorname{per}\left(\exp ^{*}(\beta)\right)\right]_{\chi} \in \mathbb{Z}_{p}^{\mathrm{ur}, \times} .
$$

Proof. If $p \nmid|G|$ then the module $H^{1}\left(K, \mathbb{Z}_{p}(1-r)\right) /$ tor is free over $\mathbb{Z}_{p}[G]$ since this is true for any lattice in a free rank-one $\mathbb{Q}_{p}[G]$-module. The first statement is then clear from (9) and Proposition 5.

Since

$$
R \Gamma\left(K, \mathbb{Z}_{p}(1-r)\right) \otimes_{\mathbb{Z}_{p}[G]}^{L} \mathbb{Z}_{p}[\Sigma] \cong R \Gamma\left(F, \mathbb{Z}_{p}(1-r)\right)
$$

the projection $\left[C_{\beta}\right]_{F}$ of $\left[C_{\beta}\right]$ into $K_{1}\left(\mathbb{Q}_{p}^{\mathrm{ur}}[\Sigma]\right) / \operatorname{im} K_{1}\left(\mathbb{Z}_{p}^{\mathrm{ur}}[\Sigma]\right)$ is the class of the complex

$$
H^{1}\left(F, \mathbb{Z}_{p}(1-r)\right)_{\text {tor }}[-1] \oplus H^{2}\left(F, \mathbb{Z}_{p}(1-r)\right)[-2]
$$

and both modules have trivial $\Sigma$-action. Any finite cyclic $\mathbb{Z}_{p}[\Sigma]$-module $M$ with trivial $\Sigma$-action has a projective resolution

$$
0 \rightarrow \mathbb{Z}_{p}[\Sigma] \stackrel{|M| e_{1}+1-e_{1}}{\longrightarrow} \mathbb{Z}_{p}[\Sigma] \rightarrow M \rightarrow 0
$$

and the class of $M$ in $K_{0}\left(\mathbb{Z}_{p}[\Sigma], \mathbb{Q}_{p}\right)$ is represented by $\left[|M| e_{1}+1-e_{1}\right]^{-1} \in$ $K_{1}\left(\mathbb{Q}_{p}[\Sigma]\right)$. Using Tate local duality we have

$$
\begin{aligned}
{\left[C_{\beta}\right]_{F} } & =\left[H^{1}\left(F, \mathbb{Z}_{p}(1-r)\right)_{\text {tor }}\right]^{-1} \cdot\left[H^{2}\left(F, \mathbb{Z}_{p}(1-r)\right)\right] \\
& =\left[H^{0}\left(F, \mathbb{Q}_{p} / \mathbb{Z}_{p}(1-r)\right)\right]^{-1} \cdot\left[H^{0}\left(F, \mathbb{Q}_{p} / \mathbb{Z}_{p}(r)\right)\right] \\
& =\left[\left(\chi^{\text {cyclo }}(\gamma)^{r-1}-1\right) e_{1}+1-e_{1}\right] \cdot\left[\left(\chi^{\text {cyclo }}(\gamma)^{r}-1\right) e_{1}+1-e_{1}\right]^{-1} \\
& =\left[\frac{\chi^{\text {cyclo }}(\gamma)^{r-1}-1}{\chi^{\text {cyclo }}(\gamma)^{r}-1} e_{1}+1-e_{1}\right] .
\end{aligned}
$$


By Proposition $5[\operatorname{per}(b)]_{\chi}$ is a $p$-adic unit if $\eta=1$, which gives the second statement. The third statement follows from the fact that $\operatorname{Gal}\left(K / K \cap F\left(\zeta_{p}\right)\right)$ acts trivially on $R \Gamma\left(K, \mathbb{Z}_{p}(1-r)\right)$ which implies that $\left[C_{\beta}\right]_{\chi}=1$ if the restriction of $\eta$ to $\operatorname{Gal}\left(K / K \cap F\left(\zeta_{p}\right)\right)$ is nontrivial.

\section{The Cherbonnier-Colmez reciprocity law}

Now that we have reformulated Equation (4) according to Proposition 7 we see that we must compute the image of $\exp ^{*}(\beta)$. In order to do this we will use an explicit reciprocity law of [Cherbonnier and Colmez 1999], which uses the theory of $\left(\varphi, \Gamma_{K}\right)$-modules and the rings of periods of Fontaine. Rather than developing this machinery in full, we will give only the definitions and results needed to state the reciprocity in our case; the reader is invited to read [Cherbonnier and Colmez 1999] to see the theory and the reciprocity law developed in full generality.

4.1. Iwasawa theory. In this subsection and the next we recall results of [Cherbonnier and Colmez 1999] specialized to the representation $V=\mathbb{Q}_{p}(1)$. For this discussion we temporarily suspend our assumption that $p \nmid|G|$. So let $K$ again be an arbitrary finite Galois extension of $\mathbb{Q}_{p}$, define

$$
\begin{gathered}
K_{n}=K\left(\zeta_{p^{n}}\right), \quad K_{\infty}=\bigcup_{n \in \mathbb{N}} K_{n}, \\
\Gamma_{K}:=\operatorname{Gal}\left(K_{\infty} / K\right), \quad \Lambda_{K}=\mathbb{Z}_{p} \llbracket \operatorname{Gal}\left(K_{\infty} / \mathbb{Q}_{p}\right) \rrbracket
\end{gathered}
$$

and

$$
H_{I w}^{m}\left(K, \mathbb{Z}_{p}(1)\right)={\underset{n}{\lim }}_{H^{m}} H^{m}\left(K_{n}, \mathbb{Z}_{p}(1)\right) \cong \underset{n}{\lim } H^{m}\left(K, \operatorname{Ind}_{G_{K_{n}}}^{G_{K}} \mathbb{Z}_{p}(1)\right) \cong H^{m}(K, T),
$$

where the inverse limit is taken with respect to corestriction maps, the second isomorphism is Shapiro's lemma and

$$
T:={\underset{\lim }{n}}_{\operatorname{lnd}_{G_{K}}}^{G_{K}} \mathbb{Z}_{p}(1) \cong{\underset{n}{\lim }}_{\mathbb{Z}_{p}}\left[\operatorname{Gal}\left(K_{n} / K\right)\right](1) \cong \mathbb{Z}_{p} \llbracket \Gamma_{K} \rrbracket(1)
$$

is a free rank-one $\mathbb{Z}_{p} \llbracket \Gamma_{K} \rrbracket$-module with $G_{K}$-action given by $\psi^{-1} \chi^{\text {cyclo }}$, where

$$
\psi: G_{K} \rightarrow \Gamma_{K} \subseteq \mathbb{Z}_{p} \llbracket \Gamma_{K} \rrbracket^{\times}
$$

is the tautological character (see the analogous discussion of (2)). From this it is easy to see that for any $r \in \mathbb{Z}$ one has an exact sequence of $G_{K}$-modules

$$
0 \rightarrow T \stackrel{\gamma_{K} \cdot \chi^{\mathrm{cyclo}}\left(\gamma_{K}\right)^{r-1}-1}{\longrightarrow} T \longrightarrow \mathbb{Z}_{p}(r) \rightarrow 0
$$


where $\gamma_{K} \in \Gamma_{K}$ is a topological generator (our assumption that $p$ is odd assures that $\Gamma_{K}$ is procyclic for any $K$ ). It is clear from the definition that

$$
H_{I w}^{m}\left(K, \mathbb{Z}_{p}(1)\right) \cong H_{I w}^{m}\left(K_{n}, \mathbb{Z}_{p}(1)\right)
$$

for any $n \geq 0$. So $H_{I w}^{m}\left(K, \mathbb{Z}_{p}(1)\right)$ only depends on the field $K_{\infty}$, and it is naturally a $\Lambda_{K}$-module. Since our base field $K$ was arbitrary an analogous sequence holds with $K$ replaced by $K_{n}$ and $T$ by the corresponding $G_{K_{n}}$-module $T_{n}$ so that $T \cong \operatorname{Ind}_{G_{K}}^{G_{K}} T_{n}$. In view of (18) we obtain induced maps

$$
\operatorname{pr}_{n, r}: H_{I w}^{1}\left(K, \mathbb{Z}_{p}(1)\right) \rightarrow H^{1}\left(K_{n}, \mathbb{Z}_{p}(r)\right)
$$

for any $n \geq 0$ and $r \in \mathbb{Z}$.

Lemma 8. Set $\gamma_{n}=\gamma_{K_{n}}$. If $r \neq 1$ then the map $\mathrm{pr}_{n, r}$ induces an isomorphism

$$
H_{I w}^{1}\left(K, \mathbb{Z}_{p}(1)\right) /\left(\gamma_{n}-\chi^{\text {cyclo }}\left(\gamma_{n}\right)^{1-r}\right) H_{I w}^{1}\left(K, \mathbb{Z}_{p}(1)\right) \cong H^{1}\left(K_{n}, \mathbb{Z}_{p}(r)\right) \text {. }
$$

Proof. The short exact sequence (17) over $K_{n}$ induces a long exact sequence of cohomology groups

$$
\begin{aligned}
& 0 \longrightarrow H_{I w}^{0}\left(K, \mathbb{Z}_{p}(1)\right) \stackrel{\gamma_{n}-\chi^{\text {cyclo }}\left(\gamma_{n}\right)^{1-r}}{\longrightarrow} H_{I w}^{0}\left(K, \mathbb{Z}_{p}(1)\right) \longrightarrow H^{0}\left(K_{n}, \mathbb{Z}_{p}(r)\right)
\end{aligned}
$$

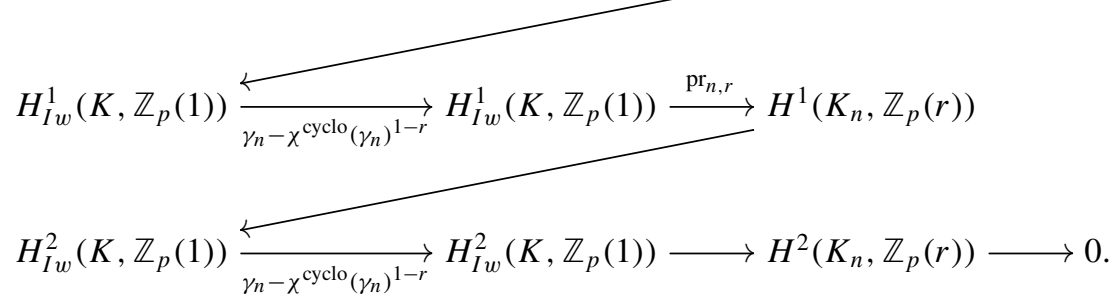

By Tate local duality there is a canonical isomorphism of $\mathrm{Gal}\left(K_{n} / K\right)$-modules

$$
H^{2}\left(K_{n}, \mathbb{Z}_{p}(1)\right) \cong \mathbb{Z}_{p}
$$

for each $n$, and the corestriction map is the identity map on $\mathbb{Z}_{p}$. Hence,

$$
H_{I w}^{2}\left(K, \mathbb{Z}_{p}(1)\right) \cong \mathbb{Z}_{p}
$$

with trivial action of $\Gamma_{K_{n}}$. This implies that for $r \neq 1$ multiplication by

$$
\gamma_{n}-\chi^{\text {cyclo }}\left(\gamma_{n}\right)^{1-r}=1-\chi^{\text {cyclo }}\left(\gamma_{n}\right)^{1-r}
$$

is injective on $H_{I w}^{2}\left(K, \mathbb{Z}_{p}(1)\right)$. Hence $\mathrm{pr}_{n, r}$ is surjective and we obtain the desired isomorphism. 
4.2. The ring $A_{K}$ and the reciprocity law. The theory of $\left(\varphi, \Gamma_{K}\right)$-modules [Cherbonnier and Colmez 1999] involves a ring

$$
A_{K}=\left(\mathcal{O}_{F^{\prime}} \llbracket\left[\pi_{K} \rrbracket\left[1 / \pi_{K}\right]\right)^{\wedge}=\left\{\sum_{n \in \mathbb{Z}} a_{n} \pi_{K}^{n}: a_{n} \in \mathcal{O}_{F^{\prime}}, \lim _{n \rightarrow-\infty} a_{n}=0\right\},\right.
$$

where $\pi_{K}$ is (for now) a formal variable and $F^{\prime} \supseteq F$ is the maximal unramified subfield of $K_{\infty}$. (The notation $(-)^{\wedge}$ means $\widehat{-}$.) The ring $A_{K}$ carries an operator $\varphi$ extending the Frobenius on $\mathcal{O}_{F^{\prime}}$ and an action of $\Gamma_{K}$ commuting with $\varphi$, which are somewhat hard to describe in terms of $\pi_{K}$. However, on the subring

$$
A_{F^{\prime}}=\left(\mathcal{O}_{F^{\prime}} \llbracket \pi \pi[1 / \pi]\right)^{\wedge} \subseteq A_{K}
$$

one has

$$
\varphi(1+\pi)=(1+\pi)^{p}, \quad \gamma(1+\pi)=(1+\pi)^{\chi^{\text {cyclo }}(\gamma)}
$$

for $\gamma \in \Gamma_{K}$.

The ring $A_{K}$ is a complete, discrete valuation ring with uniformizer $p$. We denote by $E_{K} \cong k\left(\left(\bar{\pi}_{K}\right)\right)$ its residue field and by $B_{K}=A_{K}[1 / p]$ its field of fractions. We see that $\varphi\left(B_{K}\right)$ is a subfield of $B_{K}$ (of degree $p$ ), and thus we can define

$$
\psi=p^{-1} \varphi^{-1} \operatorname{Tr}_{B_{K} / \varphi B_{K}}
$$

and

$$
\mathcal{N}=\varphi^{-1} N_{B_{K} / \varphi B_{K}}
$$

as further operators on $B_{K}$. We observe that if $f \in B_{K}$, then

$$
\psi(\varphi(f))=f .
$$

Thus $\psi$ is an additive left inverse of $\varphi$. We write $A_{K}^{\psi=1} \subset A_{K}$ for the set of elements fixed by the operator $\psi$. The $\left(\varphi, \Gamma_{K}\right)$-module associated to the representation $\mathbb{Z}_{p}(1)$ is $A_{K}(1)$ where the Tate twist refers to the $\Gamma_{K}$-action being twisted by the cyclotomic character.

By [Cherbonnier and Colmez 1999, III.2] the field $B_{K}$ is contained in a field $\widetilde{B}$ on which $\varphi$ is bijective and $\widetilde{B}$ contains a $G_{K}$-stable subring $\widetilde{B}^{\dagger, n}$ consisting of elements $x$ for which $\varphi^{-n}(x)$ converges to an element in $B_{\mathrm{dR}}$. So one has a $G_{K^{-}}$ equivariant ring homomorphism

$$
\varphi^{-n}: \widetilde{B}^{\dagger, n} \rightarrow B_{\mathrm{dR}},
$$

which again is rather inexplicit in general but is given by

$$
\varphi^{-n}(\pi)=\zeta_{p^{n}} e^{t / p^{n}}-1
$$

on the element $\pi$.

The main result [Cherbonnier and Colmez 1999, théorème IV.2.1] specialized to the representation $V=\mathbb{Q}_{p}(1)$ can now be summarized as follows. 
Theorem 9. Let $K / \mathbb{Q}_{p}$ be any finite Galois extension and

$$
\Lambda_{K}:=\mathbb{Z}_{p} \llbracket \operatorname{Gal}\left(K_{\infty} / \mathbb{Q}_{p}\right) \rrbracket
$$

its Iwasawa algebra.

(a) There is an isomorphism of $\Lambda_{K}$-modules

$$
\operatorname{Exp}_{\mathbb{Z}_{p}}^{*}: H_{I w}^{1}\left(K, \mathbb{Z}_{p}(1)\right) \cong A_{K}^{\psi=1}(1) .
$$

(b) There is $n_{0} \in \mathbb{Z}$ such that for $n \geq n_{0}$ the following hold:

(b1) $A_{K}^{\psi=1} \subseteq \widetilde{B}^{\dagger, n}$.

(b2) The $G_{K}$-equivariant map $\varphi^{-n}: A_{K}^{\psi=1} \rightarrow B_{\mathrm{dR}}$ factors through

$$
\varphi^{-n}: A_{K}^{\psi=1} \rightarrow K_{n} \llbracket t \rrbracket \subseteq B_{\mathrm{dR}}
$$

(b3) One has

$$
p^{-n} \varphi^{-n}\left(\operatorname{Exp}_{\mathbb{Z}_{p}}^{*}(u)\right)=\sum_{r=1}^{\infty} \exp _{\mathbb{Q}_{p}(r)}^{*}\left(\operatorname{pr}_{n, 1-r}(u)\right) \cdot t^{r-1}
$$

for any $u \in H_{I w}^{1}\left(K, \mathbb{Z}_{p}(1)\right)$.

Theorem 9 contains all the information we shall need when analyzing the case of tamely ramified $K$ in Section 6 below. However, the paper [Cherbonnier and Colmez 1999] contains further information on the map $\operatorname{Exp}_{\mathbb{Z}_{p}}^{*}$, which we summarize in the next proposition. We shall only need this proposition when reproving the unramified case of the local Tamagawa number in Section 5 below. First recall from [Cherbonnier and Colmez 1999, p. 257] that the ring $B_{K}$ carries a derivation

$$
\nabla: B_{K} \rightarrow B_{K}
$$

uniquely specified by its value on $\pi$ :

$$
\nabla(\pi)=1+\pi
$$

We set

$$
\nabla \log (x)=\frac{\nabla(x)}{x}
$$

and denote by

$$
\widehat{M}:=\lim _{n} M / p^{n} M
$$

the $p$-adic completion of an abelian group $M$. 
Proposition 10. There is a commutative diagram of $\Lambda_{K}$-modules, where the maps labeled by $\cong$ are isomorphism.

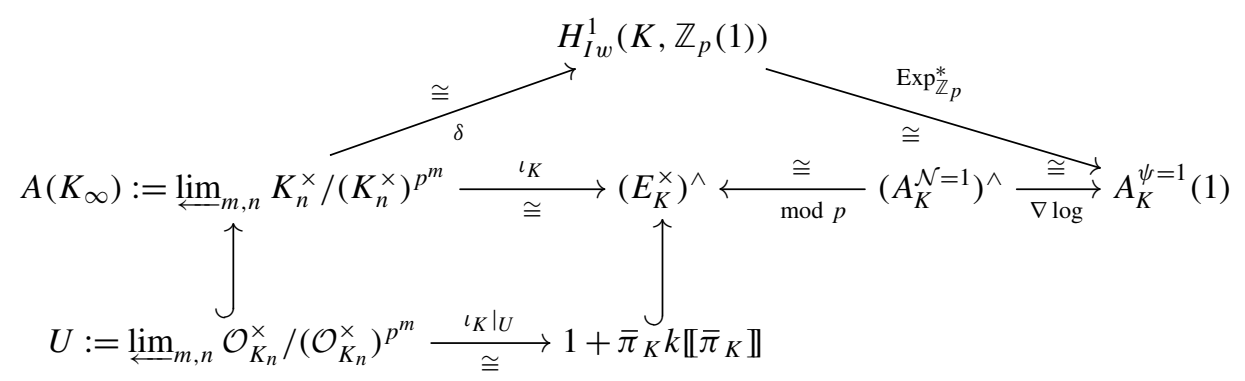

Proof. The isomorphism $\delta$ arises from Kummer theory. The theory of the field of norms gives an isomorphism of multiplicative monoids [Cherbonnier and Colmez 1999, proposition I.1.1]

$$
\lim _{n} \mathcal{O}_{K_{n}} \cong k \llbracket \bar{\pi}_{K} \rrbracket
$$

which induces our isomorphism $\left.\iota_{K}\right|_{U}$ after restricting to units and passing to $p$-adic completions and our isomorphism $\iota_{K}$ by taking the field of fractions and passing to $p$-adic completions of its units.

By [Cherbonnier and Colmez 1999, corollaire V.1.2] (see also [Daigle 2014, 3.2.1] for more details), the reduction-mod- $p$-map $\left(A_{K}^{\mathcal{N}=1}\right)^{\wedge} \rightarrow\left(E_{K}^{\times}\right)^{\wedge}$ is an isomorphism.

By [Cherbonnier and Colmez 1999, proposition V.3.2(iii)] the map $\nabla \log$ makes the upper triangle in our diagram commute. Since all other maps in this triangle are isomorphisms, the map $\nabla \log$ is an isomorphism as well.

4.3. Specialization to the tamely ramified case. We now resume our assumption that $p$ does not divide the degree of $\left[K: \mathbb{Q}_{p}\right]$ together with (most of) the notation from Section 3. In addition we assume that

$$
\zeta_{p} \in K
$$

which implies that $K_{\infty} / K$ is totally ramified and hence that $F=F^{\prime}$ is the maximal unramified subfield of $K_{\infty}$. The theory of fields of norms [Cherbonnier and Colmez 1999, remarque I.1.2] shows that $E_{K}$ is a Galois extension of $E_{F}$ of degree

$$
e:=\left[K_{\infty}: F_{\infty}\right]=\left[K: F\left(\zeta_{p}\right)\right]
$$

with group

$$
\operatorname{Gal}\left(E_{K} / E_{F}\right) \cong \operatorname{Gal}\left(K_{\infty} / F_{\infty}\right) \cong \operatorname{Gal}\left(K / F\left(\zeta_{p}\right)\right) .
$$

Note that with this notation the ramification degree of $K / \mathbb{Q}_{p}$ is $e(p-1)$ whereas it was denoted by $e$ in Section 3. The element $p_{0}$ of Section 3 we choose to be $-p$, 
i.e., we assume that

$$
K=F(\sqrt[e(p-1)]{-p})
$$

An easy computation shows that $\left(\zeta_{p}-1\right)^{p-1}=-p \cdot u$ with $u \in 1+\left(\zeta_{p}-1\right) \mathbb{Z}_{p}\left[\zeta_{p}\right]$ and hence we can choose the root $\sqrt[(p-1)]{-p}$ such that

$$
\zeta_{p}-1=\sqrt[(p-1)]{-p} \cdot u^{\prime}
$$

with $u^{\prime} \in 1+\left(\zeta_{p}-1\right) \mathbb{Z}_{p}\left[\zeta_{p}\right]$. By Kummer theory we then also have

$$
K=F\left(\sqrt[e]{\zeta_{p}-1}\right)
$$

and $B_{K}=B_{F}(\sqrt[e]{\pi})$. Any choice of $\pi_{K}=\sqrt[e]{\pi}$ fixes a choice of

$$
\sqrt[e]{\zeta_{p}-1}=\left.\varphi^{-1}\left(\pi_{K}\right)\right|_{t=0}
$$

and of

$$
\sqrt[e(p-1)]{-p}=\sqrt[e]{\zeta_{p}-1} \cdot\left(u^{\prime}\right)^{-1 / e}
$$

We have

$$
G \cong \Sigma \ltimes \Delta
$$

with $\Sigma$ cyclic of order $f$ and $\Delta$ cyclic of order $e(p-1)$ and

$$
\Lambda_{K} \cong \mathbb{Z}_{p} \llbracket G \times \Gamma_{K} \rrbracket \cong \mathbb{Z}_{p}[\Sigma \ltimes \Delta] \llbracket \gamma_{1}-1 \rrbracket,
$$

where $\gamma_{1}=\gamma^{p-1}$ is a topological generator of $\Gamma_{K}$.

Proposition 11. There is an isomorphism of $\Lambda_{K}$-modules

$$
H_{I w}^{1}\left(K, \mathbb{Z}_{p}(1)\right) \cong \Lambda_{K} \cdot \beta_{I w} \oplus \mathbb{Z}_{p}(1) .
$$

Proof. In view of the Kummer theory isomorphism

$$
\delta: A\left(K_{\infty}\right) \cong H_{I w}^{1}\left(K, \mathbb{Z}_{p}(1)\right)
$$

it suffices to quote the structure theorem for the $\Lambda_{K}$-module $A\left(K_{\infty}\right)$ given in [Neukirch et al. 2000, Theorem 11.2.3] (where $k=\mathbb{Q}_{p}$ and our group $\Sigma \ltimes \Delta$ is the group $\Delta$ of [loc. cit.]).

Corollary 12. There is an isomorphism of $\mathbb{Z}_{p}[G]$-modules

$$
H^{1}\left(K, \mathbb{Z}_{p}(1-r)\right) \cong \mathbb{Z}_{p}[G] \cdot \beta \oplus H^{1}\left(K, \mathbb{Z}_{p}(1-r)\right)_{\text {tor }},
$$

where $\beta=\operatorname{pr}_{0,1-r}\left(\beta_{I w}\right)=\operatorname{pr}_{1,1-r}\left(\beta_{I w}\right)$. 
Proof. This is clear from Proposition 11 and Lemma 8 (with $r$ replaced by $1-r$ ) in view of the isomorphisms

$$
\mathbb{Z}_{p}[G] \stackrel{\sim}{\longrightarrow} \Lambda_{K} /\left(\gamma_{1}-\chi^{\text {cyclo }}\left(\gamma_{1}\right)^{r}\right) \Lambda_{K}
$$

and

$$
\begin{aligned}
\mathbb{Z}_{p}(1) /\left(\gamma_{1}-\chi^{\mathrm{cyclo}}\left(\gamma_{1}\right)^{r}\right) \mathbb{Z}_{p}(1) & =\mathbb{Z}_{p} /\left(\chi^{\mathrm{cyclo}}\left(\gamma_{1}\right)-\chi^{\mathrm{cyclo}}\left(\gamma_{1}\right)^{r}\right) \mathbb{Z}_{p} \\
& \left.\cong H^{0}\left(K, \mathbb{Q}_{p} / \mathbb{Z}_{p}(1-r)\right)\right) \\
& \cong H^{1}\left(K, \mathbb{Z}_{p}(1-r)\right)_{\text {tor }} .
\end{aligned}
$$

If we choose the element $\beta$ of Corollary 12 to verify the identity in Proposition 7 it remains to get an explicit hold on some $\Lambda_{K}$-basis $\beta_{I w}$, or rather of its image

$$
\alpha=\operatorname{Exp}_{\mathbb{Z}_{p}}^{*}\left(\beta_{I w}\right) \in A_{K}^{\psi=1}(1) .
$$

Since $\alpha$ is a (infinite) Laurent series in $\pi_{K}$ it will be amenable to somewhat explicit analysis. In the unramified components of Proposition $7(\eta=1)$ we can compute $\alpha$ in terms of the well-known Perrin-Riou basis (see Proposition 24 below) which is a main ingredient in all known proofs of the unramified case of the local Tamagawa number conjecture. In the other components $(\eta \neq 1)$ we shall simply use Nakayama's lemma to analyze $\alpha$ as much as we can in Section 6.

In order to compute $\exp _{\mathbb{Q}_{p}(r)}^{*}(\beta)$ we also need to be able to apply Theorem 9 for $n=1$.

Proposition 13. Part (b) of Theorem 9 holds with $n_{0}=1$.

Proof. It will follow from an explicit analysis of elements in $A_{K}^{\psi=1}$ in Corollary 37 below that $\varphi^{-1}(a)$ converges for $a \in A_{K}^{\psi=1}$, which shows (b1). Since $\pi_{K}^{e}=\pi$ and $\varphi^{-n}(\pi)=\zeta_{p^{n}} e^{t / p^{n}}-1$ it is also clear that the values of $\varphi^{-n}$ on $A_{K}$, if convergent, lie in $F\left(\sqrt[e]{\zeta p^{n}-1}\right) \llbracket t \rrbracket=K_{n} \llbracket t \rrbracket$. This shows (b2). By [Cherbonnier and Colmez 1999, théorème IV.2.1] the right-hand side of (b3) is given by $T_{n} \varphi^{-m}\left(\operatorname{Exp}_{\mathbb{Z}_{p}}^{*}(u)\right)$ for $m \geq n$ large enough (see the next section for the definition of $T_{n}$ ). The statement in (b3) then follows from Corollary 17 below.

4.4. Some power series computations. The purpose of this section is simply to record some computations justifying Theorem 9(b3) for $n \geq 1$. Another aim is to write the coefficients of the right-hand side of Theorem 9(b3) in terms of the derivation $\nabla$ applied to the left-hand side. First we have

Lemma 14 [Cherbonnier and Colmez 1999, lemme III.2.3]. Suppose $\varphi^{-n} f$ and $\varphi^{-n}(\nabla f)$ both converge in $B_{\mathrm{dR}}$. Then

$$
\varphi^{-n}(\nabla f)=p^{n} \frac{d}{d t}\left(\varphi^{-n}(f)\right) .
$$


Proof. It's enough to check that $\varphi^{-n} \circ \nabla$ and $p^{n} \frac{d}{d t} \circ \varphi^{-n}$ both agree on $1+\pi$, since they are both derivations. We see that

$$
\begin{aligned}
\varphi^{-n} \nabla(1+\pi) & =\varphi^{-n}(1+\pi)=\zeta_{p^{n}} e^{t / p^{n}} \\
p^{n} \frac{d}{d t} \varphi^{-n}(1+\pi) & =p^{n} \frac{d}{d t} \zeta_{p^{n}} e^{t / p^{n}}=\zeta_{p^{n}} e^{t / p^{n}} .
\end{aligned}
$$

The next Lemma shows that $\nabla$ is compatible with other operators that we have introduced. The ring $B$ is defined as in [Cherbonnier and Colmez 1999].

Lemma 15. Let $f \in B_{K}$. Then we have

(a) $\nabla \gamma f=\chi^{\text {cyclo }}(\gamma) \cdot \gamma \nabla f$,

(b) $\nabla \varphi f=p \cdot \varphi \nabla f$,

(c) $\nabla \operatorname{Tr}_{B / \varphi B} f=\operatorname{Tr}_{B / \varphi B} \nabla f$,

(d) $\nabla \psi f=p^{-1} \cdot \psi \nabla f$.

Proof. This is a straightforward computation. For example, to see (c) note that $(1+\pi)^{i}, i=0, \ldots, p-1$ is a $\varphi B$-basis of $B$ and

$$
\operatorname{Tr}_{B / \varphi B}(x)=\operatorname{Tr}_{B / \varphi B}\left(\sum_{i=0}^{p-1} \varphi x_{i} \cdot(1+\pi)^{i}\right)=p \cdot \varphi x_{0} .
$$

Hence

$$
\begin{aligned}
\operatorname{Tr}_{B / \varphi B}(\nabla x) & =\operatorname{Tr}_{B / \varphi B}\left(\sum_{i=0}^{p-1} \nabla \varphi x_{i} \cdot(1+\pi)^{i}+\varphi x_{i} \cdot i \cdot(1+\pi)^{i}\right) \\
& =\operatorname{Tr}_{B / \varphi B}\left(\sum_{i=0}^{p-1} \varphi\left(p \nabla x_{i}+x_{i} \cdot i\right) \cdot(1+\pi)^{i}\right) \\
& =p^{2} \varphi \nabla x_{0}=\nabla\left(p \cdot \varphi x_{0}\right)=\nabla \operatorname{Tr}_{B / \varphi B}(x) .
\end{aligned}
$$

See [Daigle 2014, Lemma 3.1.3] for more details.

Recall the normalized trace maps

$$
T_{n}: K_{\infty} \rightarrow K_{n}
$$

from [Cherbonnier and Colmez 1999, p. 259] which are given by

$$
T_{n}(x)=p^{-m} \operatorname{Tr}_{K_{m} / K_{n}} x
$$

for any $m \geq n$ such that $x \in K_{m}$, and extend to a map

$$
T_{n}: K_{\infty} \llbracket t \rrbracket \rightarrow K_{n} \llbracket t \rrbracket
$$

by linearity. By [Cherbonnier and Colmez 1999, théorème IV.2.1] the right-hand side of Theorem 9(b3) is given by $T_{n} \varphi^{-m}(f)$ for $f=\operatorname{Exp}_{\mathbb{Z}_{p}}^{*}(u) \in A_{K}^{\psi=1}$ and $m \geq n$ 
large enough. In order to get access to individual Taylor coefficients of the righthand side we wish to compute $\frac{d^{r-1}}{d t^{r-1}} T_{n} \varphi^{-m}(f)$, but from Lemmas 14 and 15 we see that

$$
\frac{d^{r-1}}{d t^{r-1}} T_{n} \varphi^{-m}=p^{-m(r-1)} T_{n} \varphi^{-m} \nabla^{r-1}
$$

and thus we can study the map $T_{n} \varphi^{-m}$ on $\nabla^{r-1} A_{K}^{\psi=1}$. But since $\psi \nabla x=p \nabla \psi x$, we see that $\nabla^{r-1} A_{K}^{\psi=1} \subseteq A_{K}^{\psi=p^{r-1}}$, and so we wish to study $T_{n} \varphi^{-m}$ on $A_{K}^{\psi=p^{r-1}}$.

Lemma 16. Let $P \in A_{K}^{\psi=p^{r-1}}$ be such that

$$
\left(\varphi^{-n} P\right)(0):=\left.\varphi^{-n} P\right|_{t=0}
$$

converges and assume $m \geq n$. Then if $n \geq 1$ we have

$$
\left(T_{n} \varphi^{-m} P\right)(0)=p^{(r-1) m-r n}\left(\varphi^{-n} P\right)(0) .
$$

and if $n=0$ we have

$$
\left(T_{0} \varphi^{-m} P\right)(0)=p^{(r-1) m}\left(1-p^{-r} \sigma^{-1}\right)\left(\varphi^{-0} P\right)(0) .
$$

Proof. Since $P \in A_{K}^{\psi=p^{r-1}}$, we know that $\psi(P)=p^{r-1} P$ and thus that

$$
p^{-r} \operatorname{Tr}_{B / \varphi B}(P)=\varphi(P) .
$$

Recall that we can choose $\pi_{K}$ such that $\pi_{K}^{e}=\pi$. Then $\left\{((1+\pi) \zeta-1)^{1 / e}: \zeta \in \mu_{p}\right\}$ is the set of conjugates of $\pi_{K}$ over $\varphi(B)$ in an algebraic closure of $B$, so this gives

$$
p^{-r} \sum_{\zeta \in \mu_{p}} P\left(((1+\pi) \zeta-1)^{1 / e}\right)=P^{\sigma}\left(\left((1+\pi)^{p}-1\right)^{1 / e}\right) .
$$

Whenever $\varphi^{-(l+1)} P$ converges for some $l \in \mathbb{N}$, the operator $\left.\varphi^{-(l+1)} P\right|_{t=0}$ corresponds to setting $\pi=\zeta_{p^{l+1}}-1$ and applying $\sigma^{-(l+1)}$ to each coefficient. We get

$$
p^{-r} \sum_{\zeta \in \mu_{p}} P^{\sigma^{-(l+1)}}\left(\left(\zeta \cdot \zeta_{p^{l+1}}-1\right)^{1 / e}\right)=P^{\sigma^{-l}}\left(\left(\zeta_{p^{l}}-1\right)^{1 / e}\right)
$$

If $l \geq 1$, this simplifies to

$$
p^{-r} \operatorname{Tr}_{K_{l+1} / K_{l}} P^{\sigma^{-(l+1)}}\left(\left(\zeta_{p^{l+1}}-1\right)^{1 / e}\right)=P^{\sigma^{-l}}\left(\left(\zeta_{p^{l}}-1\right)^{1 / e}\right),
$$

and by induction, we see that for any $1 \leq n<m$,

$$
p^{m-r(m-n)} T_{n} P^{\sigma^{-m}}\left(\left(\zeta_{p^{m}}-1\right)^{1 / e}\right)=P^{\sigma^{-n}}\left(\left(\zeta_{p^{n}}-1\right)^{1 / e}\right) .
$$


Since $P^{\sigma^{-m}}\left(\left(\zeta_{p^{m}}-1\right)^{1 / e}\right)=\left(\varphi^{-m} P\right)(0)$, this proves Equation (23). If $l=0$ then Equation (25) becomes

$$
p^{-r} \sum_{\zeta \in \mu_{p}} P^{\sigma^{-1}}\left(\left(\zeta \cdot \zeta_{p}-1\right)^{1 / e}\right)=\left(\varphi^{-0} P\right)(0) .
$$

The left-hand side is now equal to

$$
p^{-r} P^{\sigma^{-1}}(0)+p^{-r} \operatorname{Tr}_{K_{1} / K_{0}} P^{\sigma^{-1}}\left(\left(\zeta_{p}-1\right)^{1 / e}\right)
$$

and we have

$$
p^{-r} \operatorname{Tr}_{K_{1} / K_{0}}\left(P^{\sigma^{-1}}\left(\left(\zeta_{p}-1\right)^{1 / e}\right)\right)=\left(1-p^{-r} \sigma^{-1}\right)\left(\varphi^{-0} P\right)(0) .
$$

By induction we get

$$
p^{m-r m} T_{0} P^{\sigma^{-m}}\left(\left(\zeta_{p^{m}}-1\right)^{1 / e}\right)=\left(1-p^{-r} \sigma^{-1}\right)\left(\varphi^{-0} P\right)(0),
$$

which proves Equation (24).

Corollary 17. If $P \in A_{K}^{\psi=1}$ is such that $\varphi^{-n} P$ converges and $m \geq n$, then we have

$$
T_{n} \varphi^{-m} P=p^{-n} \varphi^{-n} P
$$

if $n \geq 1$, and

$$
T_{0} \varphi^{-m} P=\left(1-p^{-1} \sigma^{-1}\right) \varphi^{-0} P
$$

if $n=0$.

Proof. This follows by combining Lemma 16 for all $r$.

\section{The unramified case}

In this section we reprove the local Tamagawa number conjecture (4) in the case where $K=F$ is unramified over $\mathbb{Q}_{p}$. This was first proven in [Bloch and Kato 1990] and other proofs can be found in [Perrin-Riou 1994; Benois and Berger 2008]. The proofs differ in the kind of "reciprocity law" which they employ but all proofs, including ours, use the "Perrin-Riou basis," i.e., the $\Lambda_{F}$-basis in Proposition 24 below.

5.1. An extension of Proposition 10 in the unramified case. In this section we use results of Perrin-Riou [1990] to extend the diagram in Proposition 10 to the 
diagram in Corollary 21 below. Define

$$
\begin{aligned}
\mathcal{P}_{F} & :=\left\{\sum_{n \geq 0} a_{n} \pi^{n} \in F \llbracket \pi \rrbracket: n a_{n} \in \mathcal{O}_{F}\right\}, \\
\overline{\mathcal{P}}_{F} & :=\mathcal{P}_{F} / p \mathcal{O}_{F} \llbracket \pi \rrbracket, \\
\overline{\mathcal{P}}_{F, \log }: & =\left\{f \in \overline{\mathcal{P}}_{F}:(p-\varphi)(f)=0\right\}, \\
\mathcal{P}_{F, \log }: & =\left\{f \in \mathcal{P}_{F}: \bar{f} \in \overline{\mathcal{P}}_{F, \log }\right\}=\left\{f \in \mathcal{P}_{F}:(p-\varphi)(f) \in p \mathcal{O}_{F} \llbracket \pi \rrbracket\right\}, \\
\mathcal{O}_{F} \llbracket \pi \rrbracket_{\log }: & =\left\{f \in \mathcal{O}_{F} \llbracket \pi \rrbracket^{\times}: f \bmod p \mathcal{O}_{F} \llbracket \pi \rrbracket \in 1+\pi k \llbracket \pi \rrbracket\right\} \\
& =1+(\pi, p) .
\end{aligned}
$$

Note that $\mathcal{P}_{F}$ is the space of power series in $F$ whose derivative with respect to $\pi$ lies in $\mathcal{O}_{F} \llbracket \pi \rrbracket$. Observe that the map $d \log$ is given by an integral power series, and therefore $\log \mathcal{O}_{F} \llbracket \pi \rrbracket_{\log } \subseteq \mathcal{P}_{F}$ where the logarithm map

$$
\log (1+x)=\sum_{n \geq 1}(-1)^{n-1} \frac{x^{n}}{n}
$$

is given by the usual power series. Since $\varphi$ reduces modulo $p$ to the Frobenius, i.e., to the $p$-th power map, the logarithm series in fact induces a map

$$
\log : \mathcal{O}_{F} \llbracket \pi \rrbracket_{\log } \rightarrow \mathcal{P}_{F, \log } .
$$

We wish to show that this map is an isomorphism, and to do this we first recall a couple of lemmas from [Perrin-Riou 1990].

Lemma 18 [Perrin-Riou 1990, lemme 2.1]. Let

$$
f \in 1+\pi k \llbracket \pi \rrbracket=\widehat{\mathbb{G}}_{m}(k \llbracket \pi \rrbracket)
$$

and let $\hat{f}$ be any lift of $f$ to $\mathcal{O}_{F} \llbracket \pi \rrbracket_{\log }$. Then

$$
\log (\hat{f}) \bmod p \mathcal{O}_{F} \llbracket \pi \rrbracket \in \overline{\mathcal{P}}_{F, \log }
$$

does not depend on the choice of $\hat{f}$, and the map $f \mapsto \log (\hat{f}) \bmod p \mathcal{O}_{F} \llbracket \pi \rrbracket$ is an isomorphism $\log _{k}: 1+\pi k \llbracket \pi \rrbracket \stackrel{\sim}{\longrightarrow} \overline{\mathcal{P}}_{F, \log }$.

Lemma 19 [Perrin-Riou 1990, lemme 2.2]. Let $f \in \mathcal{P}_{F, \log }$. Then the sequence $p^{m} \psi^{m}(f)$ converges to a limit $f^{\infty} \in \mathcal{P}_{F, \log }$, and we have

(1) $f^{\infty} \equiv f \bmod p \mathcal{O}_{F} \llbracket \pi \rrbracket$,

(2) $\psi\left(f^{\infty}\right)=p^{-1} f^{\infty}$,

(3) $\left(1-p^{-1} \varphi\right) f^{\infty} \in \mathcal{O}_{F} \llbracket \pi \rrbracket$,

(4) $f^{\infty}=0$ if $f \in \mathcal{O}_{F} \llbracket \pi \rrbracket$,

(5) $f^{\infty}=g^{\infty} \quad$ if $f \equiv g \bmod p \mathcal{O}_{F} \llbracket \pi \rrbracket$. 
Corollary 20. (1) The map $\log : \mathcal{O}_{F} \llbracket \pi \rrbracket \log \rightarrow \mathcal{P}_{F, \log }$ is an isomorphism.

(2) One has a commutative diagram of isomorphisms

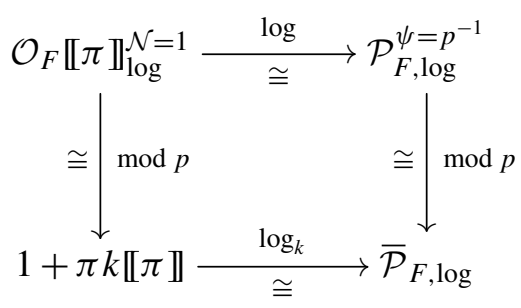

Proof. To see the first part, note that we have a commutative diagram

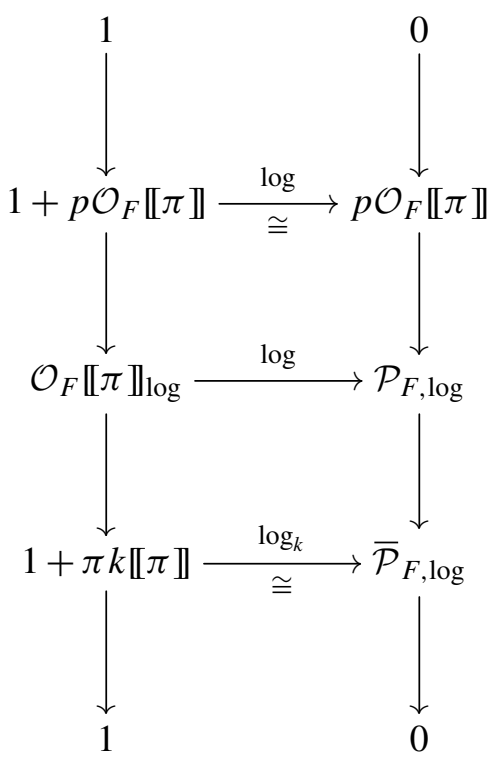

and that the logarithm map on $1+p \mathcal{O}_{F} \llbracket \pi \rrbracket$ is an isomorphism since its inverse is given by the exponential series. By the five lemma, the middle arrow is an isomorphism. For the second part, it suffices to note that Lemma 19 shows that any element in $\overline{\mathcal{P}}_{F, \log }$ has a unique lift in $\mathcal{P}_{F, \log }^{\psi=p^{-1}}$ and that $\log \mathcal{N}(x)=p \psi \log (x)$.

Corollary 21. For $K=F$ the commutative diagram from Proposition 10 extends to a commutative diagram of $\Lambda_{F}$-modules:

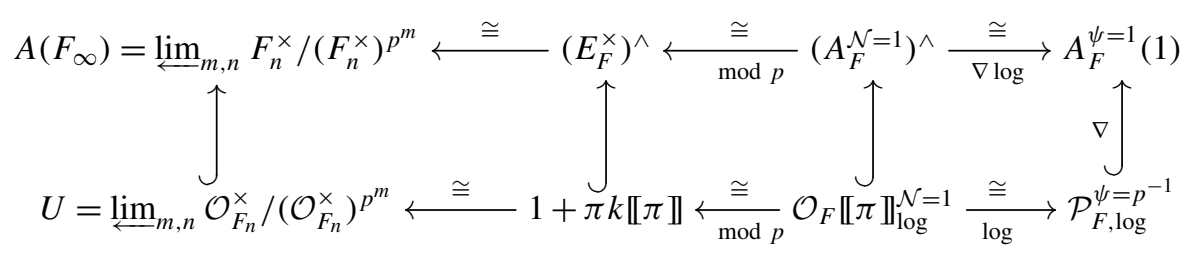

Proof. This is immediate from Corollary 20(2). 
This diagram allows us to determine the exact relationship between $\mathcal{P}_{F, \log }^{\psi=p^{-1}}$ and $A_{F}^{\psi=1}(1)$ since the relationship between $A\left(F_{\infty}\right)$ and $U$ is quite transparent. There is an exact sequence of $\Lambda_{F}$-modules

$$
0 \rightarrow U \rightarrow A\left(F_{\infty}\right) \stackrel{v}{\rightarrow} \mathbb{Z}_{p} \rightarrow 0
$$

where $v$ is the valuation map and $\mathbb{Z}_{p}$ carries the trivial $\Sigma \times \Gamma$-action. By [Neukirch et al. 2000, Theorem 11.2.3], already used in the proof of Proposition 11, there is an isomorphism

$$
A\left(F_{\infty}\right) \cong \Lambda_{F} \oplus \mathbb{Z}_{p}(1)
$$

and the torsion submodule $\mathbb{Z}_{p}(1)$ is clearly contained in $U$. Hence we obtain an exact sequence

$$
0 \rightarrow U_{\mathrm{tf}} \rightarrow A\left(F_{\infty}\right)_{\mathrm{tf}} \stackrel{v}{\rightarrow} \mathbb{Z}_{p} \rightarrow 0,
$$

where $M_{\mathrm{tf}}:=M / M_{\mathrm{tors}}$. The module $A\left(F_{\infty}\right)_{\mathrm{tf}}$ is free of rank one and since the $(\Sigma \times \Gamma)$-action on $\mathbb{Z}_{p}$ is trivial we find

$$
U_{\mathrm{tf}}=I \cdot A\left(F_{\infty}\right)_{\mathrm{tf}},
$$

where

$$
I:=(\sigma-1, \gamma-1) \subseteq \Lambda_{F}
$$

is the augmentation ideal.

Lemma 22. The augmentation ideal I is principal, generated by the element

$$
\left(1-e_{1}\right)+(\gamma-1) e_{1},
$$

where $e_{1} \in \mathbb{Z}_{p}[\Sigma]$ is the idempotent for the trivial character of $\Sigma$.

Proof. This hinges on our assumption that $p$ does not divide the order of $\Sigma$, which implies that $e_{1}$ has coefficients in $\mathbb{Z}_{p}$. Using $e_{1}^{2}=e_{1}$ we then find immediately

$$
\begin{aligned}
& \sigma-1=(\sigma-1)\left(1-e_{1}\right)=(\sigma-1)\left(1-e_{1}\right) \cdot\left[\left(1-e_{1}\right)+(\gamma-1) e_{1}\right], \\
& \gamma-1=\left((\gamma-1)\left(1-e_{1}\right)+e_{1}\right) \cdot\left[\left(1-e_{1}\right)+(\gamma-1) e_{1}\right] .
\end{aligned}
$$

Lemma 23. There are elements $\alpha \in A_{F}^{\psi=1}(1), \tilde{\alpha} \in \mathcal{P}_{F, \log }^{\psi=p^{-1}}$ such that

(1) $A_{F}^{\psi=1}(1)=\Lambda_{F} \cdot \alpha \oplus \mathbb{Z}_{p}(1) \cdot 1$,

(2) $\mathcal{P}_{F, \log }^{\psi=p^{-1}}=\Lambda_{F} \cdot \tilde{\alpha} \oplus \mathbb{Z}_{p} \cdot \log (1+\pi)$,

(3) $\nabla \tilde{\alpha}=\left(\left(1-e_{1}\right)+(\gamma-1) e_{1}\right) \cdot \alpha$. 
Proof. Part (1) follows from (27) and Corollary 21. For part (2) one checks easily that $\mathbb{Z}_{p} \cdot \log (1+\pi)$ is the torsion submodule of $\mathcal{P}_{F, \log }^{\psi=p^{-1}}$ and that $\left(\mathcal{P}_{F, \log }^{\psi=p^{-1}}\right)_{\mathrm{tf}}$ is free of rank one over $\Lambda_{F}$, since it is isomorphic under $\nabla$ to the free module

$$
I \cdot \alpha=\Lambda_{F} \cdot\left(\left(1-e_{1}\right)+(\gamma-1) e_{1}\right) \cdot \alpha
$$

by Lemma 22 . Note that we view $\alpha$ here as an element of $A_{F}(1)$, i.e., the action of $\gamma$ is $\chi^{\text {cyclo }}(\gamma)$ times the standard action (20) of $\gamma$ on $A_{F}$. Setting

$$
\tilde{\alpha}:=\nabla^{-1}\left(\left(1-e_{1}\right)+(\gamma-1) e_{1}\right) \cdot \alpha
$$

we obtain (3).

5.2. The Coleman exact sequence and the Perrin-Riou basis. Lemma 23 tells us that $\left(\mathcal{P}_{F, \log }^{\psi=p^{-1}}\right)_{\mathrm{tf}}$ is generated over $\Lambda_{F}$ by a single element $\tilde{\alpha}$, but not what this $\tilde{\alpha}$ is. By studying one more space, $\mathcal{O}_{F} \llbracket \pi \rrbracket^{\psi=0}$, we are able to describe $\tilde{\alpha}$ and hence $\alpha$.

Proposition 24. (1) There is an exact sequence of $\Lambda_{F}$-modules

$$
0 \rightarrow \mathbb{Z}_{p} \cdot \log (1+\pi) \rightarrow \mathcal{P}_{F, \log }^{\psi=p^{-1}} \stackrel{1-\varphi / p}{\longrightarrow} \mathcal{O}_{F} \llbracket \pi \rrbracket^{\psi=0} \rightarrow \mathbb{Z}_{p}(1) \rightarrow 0 .
$$

(2) $\mathcal{O}_{F} \llbracket \pi \rrbracket^{\psi=0}$ is a free $\Lambda_{F}$-module of rank one generated by $\xi(1+\pi)$, where $\xi \in \mathcal{O}_{F}$ is a basis of $\mathcal{O}_{F}$ over $\mathbb{Z}_{p}[\Sigma]$.

Proof. Part (1) is Theorem 2.3 in [Perrin-Riou 1990] and goes back to Coleman [1979]. See also [Daigle 2014, Proposition 4.1.10]. Part (2) is Lemma 1.5 in [Perrin-Riou 1990].

Corollary 25. The bases $\alpha$ and $\tilde{\alpha}$ in Lemma 23 can be chosen such that

$$
(1-\varphi / p) \cdot \tilde{\alpha}=\left(\left(1-e_{1}\right)+\left(\gamma-\chi^{\text {cyclo }}(\gamma)\right) e_{1}\right) \cdot \xi(1+\pi) .
$$

Proof. The cokernel of $(1-\varphi / p)$ in (28) is isomorphic to

$$
\mathbb{Z}_{p}(1) \cong \Lambda_{F} /\left(\sigma-1, \gamma-\chi^{\text {cyclo }}(\gamma)\right)
$$

so the image of $(1-\varphi / p)$ must be $\left(\sigma-1, \gamma-\chi^{\text {cyclo }}(\gamma)\right) \cdot \xi(1+\pi)$. As in Lemma 22 we can show that this ideal is principal, and is generated by

$$
\left(1-e_{1}\right)+\left(\gamma-\chi^{\text {cyclo }}(\gamma)\right) e_{1} .
$$

5.3. Proof of the conjecture for unramified fields. We now have the tools we need to explicitly compute $\exp _{\mathbb{Q}_{p}(r)}^{*}\left(H^{1}\left(F, \mathbb{Z}_{p}(1-r)\right)\right)$ and prove the equality of Proposition 7 for $K=F$ (i.e., $e=1$ ). By Lemma 8 we can take

$$
\beta:=\operatorname{pr}_{0,1-r}\left(\beta_{I w}\right),
$$


where $\beta_{I w}$ satisfies

$$
\begin{aligned}
\alpha & =\operatorname{Exp}_{\mathbb{Z}_{p}}^{*}\left(\beta_{I w}\right), \\
\nabla \tilde{\alpha} & =\left(\left(1-e_{1}\right)+(\gamma-1) e_{1}\right) \cdot \alpha, \\
(1-\varphi / p) \cdot \tilde{\alpha} & =\left(\left(1-e_{1}\right)+\left(\gamma-\chi^{\mathrm{cyclo}}(\gamma)\right) e_{1}\right) \cdot \xi(1+\pi),
\end{aligned}
$$

using (22), Lemma 23(3) and (29). We cannot immediately apply Theorem 9 to $n=0$, but going back to [Cherbonnier and Colmez 1999, théorème IV.2.1] we have

$$
\sum_{r=1}^{\infty} \exp _{\mathbb{Q}_{p}(r)}^{*}\left(\operatorname{pr}_{0,1-r}(u)\right) \cdot t^{r-1}=T_{0} \varphi^{-m} \operatorname{Exp}_{\mathbb{Z}_{p}}^{*}(u) .
$$

Applying this to

$$
u=\left(\left(1-e_{1}\right)+(\gamma-1) e_{1}\right) \cdot \beta_{I w}
$$

assures that

$$
\operatorname{Exp}_{\mathbb{Z}_{p}}^{*}(u)=\nabla \tilde{\alpha} \in \mathcal{O}_{F} \llbracket \pi \rrbracket
$$

and therefore

$$
\varphi^{-0} P:=\varphi^{-0} \nabla^{r-1} \operatorname{Exp}_{\mathbb{Z}_{p}}^{*}(u)=\varphi^{-0} \nabla^{r} \tilde{\alpha}
$$

converges in $B_{\mathrm{dR}}$ for any $r \geq 1$. Lemma 16 then implies

$$
\begin{aligned}
\exp _{\mathbb{Q}_{p}(r)}^{*}\left(\operatorname{pr}_{0,1-r}(u)\right) & =\left.\frac{1}{(r-1) !}\left(\frac{d}{d t}\right)^{r-1} T_{0} \varphi^{-m} \operatorname{Exp}_{\mathbb{Z}_{p}}^{*}(u)\right|_{t=0} \\
& =\left.\frac{1}{(r-1) !} T_{0} p^{-(r-1) m} \varphi^{-m} \nabla^{r-1} \operatorname{Exp}_{\mathbb{Z}_{p}}^{*}(u)\right|_{t=0} \\
& =\left.\frac{1}{(r-1) !}\left(1-p^{-r} \sigma^{-1}\right) \varphi^{-0} \nabla^{r} \tilde{\alpha}\right|_{t=0} \\
& =\left.\frac{1}{(r-1) !}\left(1-p^{-r} \sigma^{-1}\right) \nabla^{r} \tilde{\alpha}\right|_{\pi=0}
\end{aligned}
$$

Applying $\nabla^{r}$ to (29) and using Lemma 15 we have

$$
\begin{aligned}
\left(1-p^{r-1} \varphi\right) \cdot \nabla^{r} \tilde{\alpha} & =\left(\left(1-e_{1}\right)+\left(\chi^{\text {cyclo }}(\gamma)^{r} \gamma-\chi^{\text {cyclo }}(\gamma)\right) e_{1}\right) \cdot \nabla^{r} \xi(1+\pi) \\
& =\left(\left(1-e_{1}\right)+\left(\chi^{\text {cyclo }}(\gamma)^{r} \gamma-\chi^{\text {cyclo }}(\gamma)\right) e_{1}\right) \cdot \xi(1+\pi)
\end{aligned}
$$

and so we find

$$
\begin{aligned}
& \exp _{\mathbb{Q}_{p}(r)}^{*}\left(\operatorname{pr}_{0,1-r}(u)\right) \\
& \quad=\frac{1}{(r-1) !} \cdot \frac{1-p^{-r} \sigma^{-1}}{1-p^{r-1} \sigma} \cdot\left(\left(1-e_{1}\right)+\left(\chi^{\text {cyclo }}(\gamma)^{r}-\chi^{\text {cyclo }}(\gamma)\right) e_{1}\right) \cdot \xi .
\end{aligned}
$$


By Lemma 8 the action of $\gamma \in \Lambda_{F}$ on $H^{1}\left(F, \mathbb{Z}_{p}(1-r)\right)$ is via the character $\chi^{\text {cyclo }}(\gamma)^{r}$. Hence, for our choice (31) of $u$, we have

$$
\begin{aligned}
\operatorname{pr}_{0,1-r}(u) & =\left(\left(1-e_{1}\right)+\left(\chi^{\text {cyclo }}(\gamma)^{r}-1\right) e_{1}\right) \cdot \operatorname{pr}_{0,1-r}\left(\beta_{I w}\right) \\
& =\left(\left(1-e_{1}\right)+\left(\chi^{\text {cyclo }}(\gamma)^{r}-1\right) e_{1}\right) \cdot \beta
\end{aligned}
$$

and we can finally compute

$$
\exp _{\mathbb{Q}_{p}(r)}^{*}(\beta)=\frac{1}{(r-1) !} \cdot \frac{1-p^{-r} \sigma^{-1}}{1-p^{r-1} \sigma} \cdot \frac{\left(1-e_{1}\right)+\left(\chi^{\text {cyclo }}(\gamma)^{r}-\chi^{\text {cyclo }}(\gamma)\right) e_{1}}{\left(1-e_{1}\right)+\left(\chi^{\mathrm{cyclo}}(\gamma)^{r}-1\right) e_{1}} \cdot \xi
$$

This verifies the identity of Proposition 7.

\section{Results in the tamely ramified case}

We resume our notation and assumptions from Section 4.3. Our first aim in this section is to prove Proposition 44 below which is a yet more explicit reformulation of the identity (16) in Proposition 7. We then prove this identity for $e<p$ and $r=1$ as well as for $e<p / 4$ and $r=2$. In the isotypic components where $\left.\eta\right|_{\operatorname{Gal}\left(K / F\left(\zeta_{p}\right)\right)}=1$ this can easily be done (for any $r$ ) using computations similar to those in Section 5.3 with

$$
\beta_{1}:=\operatorname{pr}_{1,1-r}\left(\beta_{I w}\right)
$$

and $\beta_{I w}$ defined in (30). The notation here is relative to the base field $K=F$. In any case, the equivariant local Tamagawa number conjecture is known for any $r$ in those isotypic components by [Benois and Berger 2008]. We shall therefore entirely focus on isotypic components with

$$
\left.\eta\right|_{\operatorname{Gal}\left(K / F\left(\zeta_{p}\right)\right)} \neq 1
$$

In this case we need to verify Equation (16). The main problem is that we do not have any closed formula for a $\Lambda_{K}$-basis of (the torsion free part of) $A_{K}^{\psi=1}$. We shall analyze a general basis using Nakayama's lemma, and to do this we first need to analyze which restrictions are put on a power series

$$
a=\sum_{n} a_{n} \pi_{K}^{n} \in A_{K}
$$

by the condition $\psi(a)=a$.

6.1. Analyzing the condition $\psi=1$. Proposition 34 below, which is the main result of this subsection, gives the rate of convergence of $a_{n} \rightarrow 0$ as $n \rightarrow-\infty$ for $a \in A_{K}^{\psi=1}$. 
Definition 26. For $n \in \mathbb{N}_{0}$ and $m \in \mathbb{Z}_{(p)}$ define

$$
\begin{aligned}
b_{m, n} & :=p^{-1} \sum_{\zeta \in \mu_{p}} \zeta^{m}\left(1-\zeta^{-1}\right)^{n} \\
& =p^{-1} \operatorname{Tr}_{\mathbb{Q}\left(\zeta_{p}\right) / \mathbb{Q}} \zeta_{p}^{m}\left(1-\zeta_{p}^{-1}\right)^{n}, \quad \text { if } n \geq 1 .
\end{aligned}
$$

Clearly $b_{m, n}$ only depends on $m(\bmod p)$.

Lemma 27. One has $b_{m, n} \in \mathbb{Z}$ and

$$
b_{m, n}= \begin{cases}(-1)^{\bar{m}}\left(\begin{array}{l}
n \\
\bar{m}
\end{array}\right), & 0 \leq n<p, \\
(-1)^{\bar{m}}\left(\begin{array}{c}
n \\
\bar{m}
\end{array}\right)-(-1)^{\bar{m}}\left(\begin{array}{c}
n \\
\bar{m}+p
\end{array}\right), & p \leq n<2 p,\end{cases}
$$

where $0 \leq \bar{m}<p$ is the representative for $m(\bmod p)$. Moreover,

$$
p^{\left\lfloor\frac{n+p-2}{p-1}\right\rfloor-1} \mid b_{m, n}
$$

for $n \geq 1$ and hence

$$
p^{j} \mid b_{m, n}
$$

for $j(p-1)<n \leq(j+1)(p-1)$.

Proof. Formula (32) follows from the binomial expansion of $\left(1-\zeta^{-1}\right)^{n}$ and the fact that

$$
\sum_{\zeta \in \mu_{p}} \zeta^{k}= \begin{cases}0, & p \nmid k \\ p, & p \mid k\end{cases}
$$

In particular $b_{m, 0}=0,1$ according to whether $p \nmid m$ or $p \mid m$. The different of the extension $\mathbb{Q}\left(\zeta_{p}\right) / \mathbb{Q}$ is $\left(1-\zeta_{p}\right)^{p-2}$, so we have

$$
\begin{aligned}
& \operatorname{Tr}_{\mathbb{Q}\left(\zeta_{p}\right) / \mathbb{Q}}\left(\zeta_{p}^{m}\left(1-\zeta_{p}^{-1}\right)^{n}\right) \subseteq p^{N} \mathbb{Z} \\
& \Longleftrightarrow\left(\left(1-\zeta_{p}\right)^{n}\right) \subseteq\left(p^{N}\left(1-\zeta_{p}\right)^{2-p}\right)=\left(\left(1-\zeta_{p}\right)^{N(p-1)+2-p}\right) \\
& \Longleftrightarrow n \geq N(p-1)+2-p \Longleftrightarrow N \leq \frac{n+p-2}{p-1} .
\end{aligned}
$$

Definition 28. Define integers $\beta_{n, j} \in \mathbb{Z}$ by $\beta_{1, j}:=\frac{1}{p}\left(\begin{array}{l}p \\ j\end{array}\right)$ for $1 \leq j \leq p-1$ and

$$
\left(\sum_{j=1}^{p-1} \beta_{1, j} x^{j}\right)^{n}=\sum_{j=n}^{n(p-1)} \beta_{n, j} x^{j}
$$

Proposition 29. An element $a=\sum_{i} a_{i} \pi_{K}^{i} \in A_{K}$ lies in $A_{K}^{\psi=1}$ if and only if for all $N \in \mathbb{Z}$ one has

$$
\sum_{n=0}^{\infty} a_{N+e n}\left(\begin{array}{c}
\frac{N}{e}+n \\
n
\end{array}\right) b_{(N / e)+n, n}=\sum_{0 \leq n \leq j \leq n(p-1)} a_{(N+j e) / p}^{\sigma}\left(\begin{array}{c}
\frac{N+j e}{p e} \\
n
\end{array}\right) \beta_{n, j} \cdot p^{n}
$$


with the convention that $a_{r}=0$ for $r \notin \mathbb{Z}$. Equation (33) holds for all $N \in \mathbb{Z}$ if and only if it holds for all $N \in p \mathbb{Z}$.

Proof. This is just comparing coefficients in the identity $p^{-1} \operatorname{Tr}_{B / \varphi(B)}(a)=\varphi(a)$. One has $\varphi(\pi)=(1+\pi)^{p}-1=\pi^{p}(1+p \cdot y)$ with $y=\sum_{j=1}^{p-1} \beta_{1, j} \pi^{-j}$ and hence

$$
\varphi\left(\pi_{K}\right)=\pi_{K}^{p} \cdot \lambda \cdot(1+p \cdot y)^{1 / e}
$$

with $\lambda \in \mu_{e}$ and $(1+Z)^{1 / e}$ the binomial series. In fact, $\lambda=1$ since $\varphi\left(\pi_{K}\right) \equiv \pi_{K}^{p}$ $\bmod p$. Therefore

$$
\begin{aligned}
\varphi\left(\pi_{K}^{m}\right) & =\pi_{K}^{p m}(1+p \cdot y)^{m / e}=\pi_{K}^{p m} \sum_{n=0}^{\infty}\left(\begin{array}{c}
\frac{m}{e} \\
n
\end{array}\right) y^{n} \cdot p^{n} \\
& =\sum_{n=0}^{\infty}\left(\begin{array}{c}
\frac{m}{e} \\
n
\end{array}\right) \sum_{j=n}^{n(p-1)} \beta_{n, j} \pi_{K}^{p m-e j} \cdot p^{n}
\end{aligned}
$$

and the coefficient of $\pi_{K}^{N}$ in $\varphi(a)=\sum_{m} a_{m}^{\sigma} \varphi\left(\pi_{K}^{m}\right)$ is

$$
\sum_{m, n, j, N=p m-e j} a_{m}^{\sigma}\left(\begin{array}{c}
\frac{m}{e} \\
n
\end{array}\right) \beta_{n, j} \cdot p^{n}
$$

which is the right-hand side of (33). The conjugates of $\pi$ over $\varphi(B)$ are

$$
(1+\pi) \zeta-1=\pi \cdot \zeta \cdot\left(1+\left(1-\zeta^{-1}\right) \pi^{-1}\right),
$$

hence the conjugates of $\pi_{K}^{m}$ are

$$
\pi_{K}^{m} \cdot \zeta^{m / e} \cdot\left(1+\left(1-\zeta^{-1}\right) \pi^{-1}\right)^{m / e}=\pi_{K}^{m} \cdot \zeta^{m / e} \cdot \sum_{n=0}^{\infty}\left(\begin{array}{c}
\frac{m}{e} \\
n
\end{array}\right)\left(1-\zeta^{-1}\right)^{n} \pi^{-n}
$$

and

$$
p^{-1} \operatorname{Tr}_{B / \varphi(B)}\left(\pi_{K}^{m}\right)=\pi_{K}^{m} \cdot \sum_{n=0}^{\infty}\left(\begin{array}{c}
\frac{m}{e} \\
n
\end{array}\right) b_{m / e, n} \pi^{-n}=\sum_{n=0}^{\infty}\left(\begin{array}{c}
\frac{m}{e} \\
n
\end{array}\right) b_{m / e, n} \pi_{K}^{m-e n},
$$

and the coefficient of $\pi_{K}^{N}$ in $p^{-1} \operatorname{Tr}_{B / \varphi(B)}(a)$ is the left-hand side of (33). Note here that $B(\zeta) / \varphi(B)$ is totally ramified, so all the conjugates must be congruent modulo $1-\zeta$.

Denote by (33) $)_{m}$ the equation (33) modulo $p^{m}$. By Lemma 30 below, (33) for all $N \in \mathbb{Z}$ is equivalent to (33) $)_{1}$ for all $N \in p \mathbb{Z}$. We shall show by induction on $m$ that this equivalence holds for all $m$. Suppose $a \in A_{K}$ satisfies (33) s $_{m+1}$ for all $N \in p \mathbb{Z}$. Let $b \in A_{K}^{\psi=1}$ be a lift of $\bar{a} \in E_{K}^{\psi=1}$, which exists by Lemma 32 below, and write $a-b=c \cdot p$. Then $a-b$ satisfies (33) $)_{m+1}$ for all $N \in p \mathbb{Z}$, hence $c$ satisfies (33) ${ }_{m}$ for all $N \in p \mathbb{Z}$. By the induction assumption $c$ satisfies (33) $)_{m}$ for all $N \in \mathbb{Z}$. But then $p \cdot c$ satisfies (33) $)_{m+1}$ for all $N \in \mathbb{Z}$, hence so does $a=b+c \cdot p$. 
Lemma 30. An element $a=\sum_{i} a_{i} \pi_{K}^{i} \in E_{K}$ lies in $E_{K}^{\psi=1}$ if and only iffor all $k \in \mathbb{Z}$ one has

$$
\sum_{n=0}^{p-1} a_{k p+n e}(-1)^{n}=a_{k}^{\sigma}
$$

Proof. The only nonzero term on the right-hand side of $(33)_{1}$ is $a_{N / p}^{\sigma}$, corresponding to $n=j=0$, and the nonzero terms on the left-hand side are for $n \leq p-1$ by Lemma 27. For $m \in \mathbb{Z}_{(p)}$ one has

$$
\left(\begin{array}{l}
m \\
n
\end{array}\right)\left(\begin{array}{l}
n \\
\bar{m}
\end{array}\right)=\frac{m(m-1) \cdots(m-n+1)}{n !} \cdot \frac{n !}{\bar{m} !(n-\bar{m}) !} \equiv \begin{cases}0, & \bar{m}<n, \\
1, & \bar{m}=n,\end{cases}
$$

since for $\bar{m}<n$ one of the factors in $m(m-1) \cdots(m-n+1)$ is divisible by $p$, whereas for $\bar{m}=n$ this product is congruent to $\bar{m}$ ! modulo $p$. For $\bar{m}>n$ one has $\left(\begin{array}{l}n \\ \bar{m}\end{array}\right)=0$, so $\left(\begin{array}{c}m \\ n\end{array}\right)\left(\begin{array}{l}n \\ \bar{m}\end{array}\right) \equiv 0$ whenever $\bar{m} \neq n$. Using (32) the left-hand side of $(33)_{1}$ is

$$
\sum_{n=0}^{p-1} a_{N+e n}\left(\begin{array}{l}
m \\
n
\end{array}\right)\left(\begin{array}{l}
n \\
\bar{m}
\end{array}\right)(-1)^{\bar{m}}
$$

for $m=(N / e)+n$. So the left-hand side vanishes for $N \notin p \mathbb{Z}$ and is equal to the left-hand side of (34) for $N=p k$.

For later reference we also record here a more explicit version of $(33)_{2}$.

Lemma 31. Let $H_{0}=0$ and $H_{n}=\sum_{i=1}^{n} 1 / i$ be the harmonic number. Then (33) holds if and only if for all $k \in \mathbb{Z}$ one has

$$
\sum_{n=0}^{p-1} a_{k p+n e}(-1)^{n}\left(1+\frac{k p}{e} H_{n}\right)+\sum_{n=p+1}^{2(p-1)} a_{k p+n e}(-1)^{n-p} \cdot p \cdot H_{n-p}\left(1+\frac{k}{e}\right) \equiv a_{k}^{\sigma} \text {. }
$$

Proof. The only nonzero term on the right-hand side of $(33)_{2}$ for $N=k p$ is $a_{k}^{\sigma}$, corresponding to $n=j=0$, since for $n=1$ there is no $1 \leq j \leq(p-1)$ with $p \mid(N+j e)=k p+j e$. The nonzero terms on the left-hand side are for $n \leq 2(p-1)$ by Lemma 27 . Note that for $1 \leq j \leq n<2 p$ only $j=p$ is divisible by $p$. So computing modulo $p^{2}$ we have

$$
\begin{aligned}
\left(\begin{array}{c}
\frac{k p}{e}+n \\
n
\end{array}\right) & =\frac{\prod_{j=1}^{n}\left(\frac{k p}{e}+j\right)}{n !} \equiv \frac{n !+\frac{k p}{e} \sum_{j=1}^{n} \frac{n !}{j}+\left(\frac{k p}{e}\right)^{2} \sum_{1 \leq j_{1}<j_{2} \leq n} \frac{n !}{j_{1} j_{2}}}{n !} \\
& \equiv 1+\frac{k p}{e} H_{n}+\left(\frac{k p}{e}\right)^{2} \sum_{1 \leq j_{1}<j_{2} \leq n} \frac{1}{j_{1} j_{2}} \\
& \equiv \begin{cases}1+\frac{k p}{e} H_{n}, & n<p, \\
1+\frac{k}{e}+\frac{k p}{e} H_{n-p}+\left(\frac{k}{e}\right)^{2} \cdot p \cdot H_{n-p}, & p \leq n<2 p .\end{cases}
\end{aligned}
$$


Here we have used $H_{p-1} \equiv 0 \bmod p$ and $\sum_{j=p+1}^{n} 1 / j \equiv H_{n-p} \bmod p$. By (32) we have

$$
b_{(k p / e)+n, n}= \begin{cases}\left(\begin{array}{l}
n \\
n
\end{array}\right)(-1)^{n}=(-1)^{n}, & n<p, \\
0, & n=p, \\
(-1)^{n-p}\left(\left(\begin{array}{c}
n \\
n-p
\end{array}\right)-\left(\begin{array}{l}
n \\
n
\end{array}\right)\right), & p<n<2 p\end{cases}
$$

and

$$
\left(\begin{array}{c}
n \\
n-p
\end{array}\right)-\left(\begin{array}{l}
n \\
n
\end{array}\right)=\frac{(p+n-p)(p+n-p-1) \cdots(p+1)}{(n-p) !}-1 \equiv p \cdot \sum_{j=1}^{n-p} \frac{1}{j} .
$$

So the summand for $n=p$ vanishes and for $p<n<2 p$ we have

$$
\begin{aligned}
\left(\begin{array}{c}
\frac{k p}{e}+n \\
n
\end{array}\right) b_{(k p / e)+n, n} & \equiv\left(1+\frac{k}{e}+\frac{k p}{e} H_{n-p}+\left(\frac{k}{e}\right)^{2} \cdot p \cdot H_{n-p}\right)(-1)^{n-p} \cdot p \cdot H_{n-p} \\
& \equiv(-1)^{n-p}\left(1+\frac{k}{e}\right) \cdot p \cdot H_{n-p} .
\end{aligned}
$$

Lemma 32. The map $A_{K}^{\psi=1} \rightarrow E_{K}^{\psi=1}$ is surjective.

Proof. This follows from the snake lemma applied to

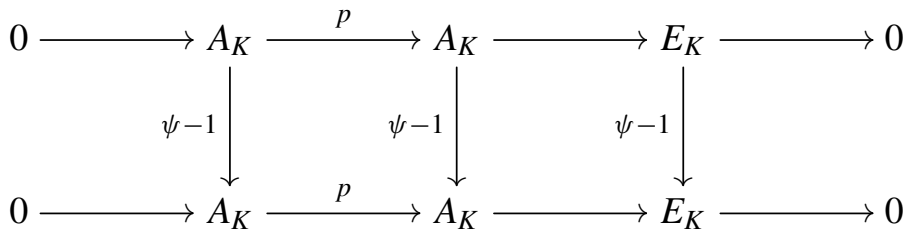

and the fact that $A_{K} /(\psi-1) A_{K} \cong H_{I w}^{2}\left(K, \mathbb{Z}_{p}(1)\right) \cong \mathbb{Z}_{p}$ (see [Cherbonnier and Colmez 1999, remarque II.3.2.]) is $p$-torsion free.

Definition 33. For $a=\sum_{i} a_{i} \pi_{K}^{i} \in A_{K}$ and $v \geq 1$ we set

$$
l_{v}(a):=\min \left\{i \mid p^{\nu} \nmid a_{i}\right\} .
$$

In particular

$$
l(a):=l_{1}(a)=v_{\pi_{K}}(\bar{a})
$$

is the valuation of $\bar{a} \in E_{K}$.

Note that $l(a)$ is independent of a choice of uniformizer for $A_{K}$, but for $v \geq 2$, $l_{v}(a)$ is not.

Proposition 34. Let $a \in A_{K}^{\psi=1}$.

(a) For all $v \geq 1$ we have

$$
l_{\nu}(a) \geq-\frac{v(p-1)+1}{p} \cdot e .
$$


In particular $l(a) \geq-e$.

(b) If $l(a)<-e+e(p-1)$ then

$$
l_{2}(a)>l(a)-e(p-1),
$$

while if $l(a) \geq-e+e(p-1)$ then $l_{2}(a) \geq-e$.

(c) If $l(a)<-e+2 e(p-1)$ and $l_{2}(a) \geq l(a)-e(p-1)$ then

$$
l_{3}(a)>l(a)-2 e(p-1),
$$

while if $l(a) \geq-e+2 e(p-1)$ and $l_{2}(a) \geq l(a)-e(p-1)$ then $l_{3}(a) \geq-e$.

Remark 35. Part (b) is a small improvement of part (a) for $v=2$ and $a$ with

$$
l(a)>-\left(2-\frac{1}{p}\right) e+e(p-1),
$$

while part (c) improves (a) for $v=3$ and $a$ with

$$
l(a)>-\left(3-\frac{2}{p}\right) e+2 e(p-1)
$$

and $l_{2}(a) \geq l(a)-e(p-1)$.

Proof. Suppose $a=\sum_{i} a_{i} \pi_{K}^{i} \in A_{K}^{\psi=1}$. Part (a) is equivalent to the statement

$$
i<-\frac{v(p-1)+1}{p} \cdot e \Rightarrow p^{v} \mid a_{i},
$$

which we denote by $(36)_{v}$ if we want to emphasize dependence on $v$. We shall prove (36) $v$ by induction on $v$, the statement $(36)_{0}$ being trivial. Now assume (36) $)_{v^{\prime}}$ for $v^{\prime} \leq v$ and assume $p^{v+1} \nmid a_{i}$ for some

$$
i<-\frac{(\nu+1)(p-1)+1}{p} \cdot e .
$$

We shall show that there is another $i^{\prime}<i$ with $p^{\nu+1} \nmid a_{i^{\prime}}$. Hence there are infinitely many $i<0$ with $p^{v+1} \nmid a_{i}$ which contradicts the fact that $a \in A_{K}$. This proves $(36)_{v+1}$.

In order to find $i^{\prime}$ we look at (33) for $N=p i$

$$
\sum_{n=0}^{\infty} a_{p i+e n}\left(\begin{array}{c}
\frac{p i}{e}+n \\
n
\end{array}\right) b_{(p i / e)+n, n}=a_{i}^{\sigma}+\sum_{1 \leq n \leq p \lambda \leq n(p-1)} a_{i+\lambda e}^{\sigma}\left(\begin{array}{c}
\frac{i}{e}+\lambda \\
n
\end{array}\right) \beta_{n, p \lambda} \cdot p^{n}
$$

and first notice that

$$
p^{v+1-n} \mid a_{i+\lambda e}
$$


for $n / p \leq \lambda \leq n(p-1) / p$. This is because of

$$
i+\lambda e<-\frac{(v+1)(p-1)+1}{p} \cdot e+\frac{n(p-1)}{p} \cdot e=-\frac{(v+1-n)(p-1)+1}{p} \cdot e
$$

and the induction assumption. Since $\left(\begin{array}{c}(i / e)+\lambda \\ n\end{array}\right) \beta_{n, p \lambda}$ is a $p$-adic integer we conclude that $p^{v+1}$ divides the sum over $\lambda, n$ in the right-hand side of (37) and hence does not divide the right-hand side of (37).

Considering the left-hand side of (37) we first recall that Lemma 27 implies that

$$
p^{j} \mid b_{(p i / e)+n, n}
$$

for $j(p-1)<n \leq(j+1)(p-1)$. For $n$ in this range we have

$$
\begin{aligned}
p i+n e & \leq p i+(j+1)(p-1) e<-((v+1)(p-1)+1) e+(j+1)(p-1) e \\
& =-((v+1-j)(p-1)+1) e+(p-1) e \\
& \leq-\frac{(v+1-j)(p-1)+1}{p} \cdot e
\end{aligned}
$$

provided this last inequality holds which is equivalent to

$$
\begin{aligned}
p((v+ & 1-j)(p-1)+1)-p(p-1) \geq(v+1-j)(p-1)+1 \\
& \Longleftrightarrow(p-1)((v+1-j)(p-1)+1) \geq p(p-1) \\
& \Longleftrightarrow((v+1-j)(p-1)+1) \geq p \\
& \Longleftrightarrow(v+1-j) \geq 1 \Longleftrightarrow v \geq j .
\end{aligned}
$$

So for $1 \leq j \leq v$ inequality (39) holds, and the induction assumption implies

$$
p^{v+1-j} \mid a_{p i+n e} .
$$

Using (38) we conclude that $p^{v+1}$ divides all summands in the left-hand side of (37) except perhaps those with $n<p$ (corresponding to $j=0$ ). Since $p^{v+1}$ does not divide the right-hand side, it does not divide the left-hand side of (37). So there must be one summand with $n<p$ not divisible by $p^{v+1}$ and hence some $i^{\prime}:=p i+e n$ with $n \leq p-1$ such that $p^{v+1} \nmid a_{i^{\prime}}$. It remains to remark that

$$
i^{\prime}=p i+e n \leq p i+e(p-1)<p i-i(p-1)=i
$$

since $i<-e$.

To prove (b) we use the same argument. Assuming the existence of

$$
i \leq \min \{l(a)-e(p-1),-e-1\}
$$

with $p^{2} \nmid a_{i}$ we find another $i^{\prime}<i$ with $p^{2} \nmid a_{i^{\prime}}$. On the right-hand side of (37), apart from $a_{i}^{\sigma}$, all summands are divisible by $p^{2}$ (note there are none with $n=1$ since $\lambda$ 
has to be an integer). On the left-hand side, summands for $n>2(p-1)$ are divisible by $p^{2}$ by Lemma 27. For $p \leq n \leq 2(p-1)$ we have, assuming $l(a)<-e+e(p-1)$,

$$
\begin{aligned}
p i+e n & \leq(l(a)-e(p-1))+2(p-1) e=l(a)+(p-1) l(a)-(p-2)(p-1) e \\
& <l(a)+(p-1)(-e+e(p-1))-(p-2)(p-1) e=l(a)
\end{aligned}
$$

and therefore $p \mid a_{p i+e n}$. If $l(a) \geq-e+e(p-1)$ we have

$$
p i+e n<p(-e)+2(p-1) e=-e+e(p-1) \leq l(a)
$$

and again conclude $p \mid a_{p i+e n}$. So all summands on the left-hand side with $n \geq p$ are divisible by $p^{2}$. Hence some $i^{\prime}:=p i+e n$ with $n \leq p-1$ satisfies $p^{2} \nmid a_{i^{\prime}}$. Moreover, (40) holds since $i<-e$.

For (c) we use this argument yet another time. Assume

$$
i \leq \min \{l(a)-2 e(p-1),-e-1\}
$$

and $p^{3} \nmid a_{i}$. On the right-hand side of (37) we need $p \mid a_{i+\lambda e}$ for $2 / p \leq \lambda \leq 2(p-1) / p$, i.e., $\lambda=1$. But

$$
i+e \leq \min \{l(a)-2 e(p-1)+e,-1\}<l(a),
$$

so $p \mid a_{i+e}$. Assume first $l(a)<-e+2 e(p-1)$. On the left-hand side we have for $p \leq n \leq 2(p-1)$

$$
\begin{aligned}
p i+e n & \leq p(l(a)-2 e(p-1))+2(p-1) e \\
& =l(a)-e(p-1)+(p-1) l(a)+e(p-1)-(2 p-2)(p-1) e \\
& <l(a)-e(p-1)+(p-1)(-e+2 e(p-1))-(2 p-3)(p-1) e \\
& =l(a)-e(p-1) \leq l_{2}(a)
\end{aligned}
$$

and therefore $p^{2} \mid a_{p i+e n}$. For $2 p-1 \leq n \leq 3(p-1)$ we just add $(p-1) e$ to this last estimate to conclude

$$
\begin{aligned}
p i+e n & \leq p(l(a)-2 e(p-1))+3(p-1) e \\
& <l(a)-e(p-1)+e(p-1)=l(a)
\end{aligned}
$$

and hence $p \mid a_{p i+e n}$. Now assume $l(a) \geq-e+2 e(p-1)$. For $p \leq n \leq 2(p-1)$ we have

$$
p i+e n \leq p(-e)+2(p-1) e \leq l(a)-e(p-1) \leq l_{2}(a)
$$

and therefore $p^{2} \mid a_{p i+e n}$. For $2 p-1 \leq n \leq 3(p-1)$ we again add $(p-1) e$ to this last estimate to conclude $p i+e n<l(a)$ and $p \mid a_{p i+e n}$. As before we conclude that, for some $i^{\prime}:=p i+e n$ with $n \leq p-1$, we have $p^{3} \nmid a_{i^{\prime}}$. Moreover (40) holds since $i<-e$. 
Before drawing consequences of Proposition 34 we make the following definition.

Definition 36. Let $\varpi$ be the uniformizer of $K$ given by

$$
\varpi=\sqrt[e]{\zeta_{p}-1}=\left.\varphi^{-1}\left(\pi_{K}\right)\right|_{t=0}
$$

and denote by $v_{\varpi}$ the unnormalized valuation of the field $K$, i.e.,

$$
v_{\varpi}(p)=e(p-1) .
$$

For $a \in B_{K}^{\dagger, 1}$ define

$$
v_{\varpi}(a):=v_{\varpi}\left(\left.\varphi^{-1}(a)\right|_{t=0}\right) .
$$

Corollary 37. For all $a \in A_{K}^{\psi=1}$ the series $\varphi^{-1}(a)$ converges, i.e., $A_{K}^{\psi=1} \subseteq B_{K}^{\dagger, 1}$. Proof. By (a) we have $p^{v} \mid a_{i}$ for

$$
-\frac{(v+1)(p-1)+1}{p} \cdot e \leq i<-\frac{v(p-1)+1}{p} \cdot e
$$

and hence

$$
v_{p}\left(a_{i}\right) \geq v \geq-\frac{i p+e}{e(p-1)}-1
$$

and

$$
v_{\varpi}\left(a_{i} \varpi^{i}\right) \geq-(i p+e)-e(p-1)+i=-(p-1) i-p e .
$$

This implies

$$
\lim _{i \rightarrow-\infty} v_{\varpi}\left(a_{i} \varpi^{i}\right)=\infty
$$

and hence the series $\sum_{i \in \mathbb{Z}} a_{i} \varpi^{i}$ converges in $K \subseteq \widehat{\overline{\mathbb{Q}}}_{p}$. By [Colmez 1999, proposition II.25] this implies that $\varphi^{-1}(a)$ converges in $B_{\mathrm{dR}}$.

Proposition 38. For each $a \in E_{K}^{\psi=1}$ we have $l(a) \geq-e$. If $l(a)>-e$ then $l(a) \not \equiv-e$ $\bmod p$. Conversely, for each $c \in k^{\times}$and $n \in \mathbb{Z}$ with

$$
-e<n \not \equiv-e \bmod p
$$

there is an element $a \in E_{K}^{\psi=1}$ with $l(a)=n$ and leading coefficient $c$.

Proof. That $l(a) \geq-e$ is Proposition 34(a). Assume that $l(a)>-e$ and $l(a) \equiv-e$ $\bmod p$. Then $l(a)=k p+(p-1) e$ for some $k \in \mathbb{Z}$ and

$$
k=\frac{l(a)-(p-1) e}{p}=l(a)-\left(1-\frac{1}{p}\right)(l(a)+e)<l(a),
$$

so we have $a_{k}=0$. Further, $a_{k p+i e}=0$ for $i=0, \ldots, p-2$ since $k p+i e<l(a)$. Hence there is only one nonzero term in (34) which gives a contradiction. 
To show the second part one can solve (34) by an easy recursion. Alternatively, Proposition 10 implies that $\nabla \log (a) \in E_{K}^{\psi=1}$ for any $a \in E_{K}^{\times}$. Now compute

$$
\nabla \log \left(1+c \pi_{K}^{n}\right)=\frac{\nabla\left(1+c \pi_{K}^{n}\right)}{1+c \pi_{K}^{n}}=\frac{c n / e \cdot\left(\pi_{K}^{n-e}+\pi_{K}^{n}\right)}{1+c \pi_{K}^{n}}=\frac{c n}{e} \cdot \pi_{K}^{n-e}+\cdots
$$

and note that for $p \nmid n$ one can produce any leading coefficient.

Remark 39. Elements $a \in E_{K}^{\psi=1}$ with $l(a)=-e$ exist, e.g.,

$$
\nabla \log \left(\pi^{j}\right)=j \cdot \pi^{-1}+j=j \cdot \pi_{K}^{-e}+j,
$$

but their leading coefficient is restricted to elements in $\mathbb{F}_{p}$.

Corollary 40. If $a \in A_{K}^{\psi=1}$ and

$$
l(a)<-e+e(p-1),
$$

we have $v_{\varpi}(a)=l(a)$.

Proof. Since $v_{\varpi}\left(a_{l(a)} \varpi^{l(a)}\right)=l(a)$ we need to show

$$
v_{\varpi}\left(a_{i} \varpi^{i}\right)>l(a)
$$

for $i \neq l(a)$. This is clear for $i>l(a)$, and also for

$$
l(a)-e(p-1)<i<l(a)
$$

since in that range $p \mid a_{i}$ and so $v_{\varpi}\left(a_{i} \varpi^{i}\right) \geq e(p-1)+i>l(a)$. For

$$
l(a)-2 e(p-1)<i \leq l(a)-e(p-1)
$$

we have $p^{2} \mid a_{i}$ by part (b) and hence $v_{\varpi}\left(a_{i} \varpi^{i}\right) \geq 2 e(p-1)+i>l(a)$. Finally for

$$
i \leq l(a)-2 e(p-1)<-e-e(p-1)=-e p<-2 e
$$

we have by (41)

$$
v_{\varpi}\left(a_{i} \varpi^{i}\right) \geq-(p-1) i-p e>(p-1) 2 e-p e=(p-2) e>l(a),
$$

using the assumption on $l(a)$.

In order to study $v_{\varpi}(a)$ for $a \in A_{K}^{\psi=1}$ with $l(a)>-e+e(p-1)$ we need to use Lemma 31 . The next proposition will show that $v_{\varpi}(a)$ cannot only depend on $l(a)$ in this case. In the situation of Proposition 41(b) one can have $v_{\varpi}(a)=l(a)$ but for any $b \in A_{K}^{\psi=1}$ with $l(b)<l(a)-e(p-1)$ and $p^{2} \nmid a_{l(b)}+p b_{l(b)}$ one has

$$
l(a+p b)=l(a), \quad v_{\varpi}(a+p b) \leq l(b)+e(p-1)<l(a)=v_{\varpi}(a) .
$$


Proposition 41. Let $a^{\prime} \in A_{K}^{\psi=1}$ with

$$
l\left(a^{\prime}\right)=\mu p-e+e(p-1)
$$

for some $\mu \in \mathbb{Z}$ with $1 \leq \mu<\frac{e(p-1)}{p}$.

(a) There exists $a \equiv a^{\prime} \bmod p$ with

$$
l_{2}(a) \geq \mu p-e=l(a)-e(p-1) .
$$

(b) For a as in (a) we have $v_{\varpi}(a) \geq l(a)$ with equality if $p \nmid \mu-e$. This last condition is automatic for $e<p$.

Proof. First note that $l_{2}\left(a^{\prime}\right) \geq-e$ by Proposition 34(b). If $l_{2}\left(a^{\prime}\right)=-e$ then Equation (35) for $k:=-e$ reads

$$
{a^{\prime}}_{-e}^{\sigma} \equiv a_{k p+e(p-1)}^{\prime}=a_{-e}^{\prime}
$$

since $i=k p+e n<l_{2}\left(a^{\prime}\right)$ for $n<p-1$ and $i=k p+e n \leq-e+e(p-1)<l\left(a^{\prime}\right)$ for $p+1 \leq n \leq 2(p-1)$. Hence $a_{-e}^{\prime} / p \bmod p \in \mathbb{F}_{p}$. Adding an element $p b$ to $a^{\prime}$, where $b$ with $l(b)=-e$ is as in Remark 39, we can assume that $l_{2}\left(a^{\prime}\right)>-e$. More generally, as long as $l_{2}\left(a^{\prime}\right)<l\left(a^{\prime}\right)$, we can add elements $p b$ to $a^{\prime}$ whose existence is guaranteed by Proposition 38 and increase $l_{2}\left(a^{\prime}\right)$ until $l_{2}\left(a^{\prime}\right)$ is not one of the possible $l(b)$, i.e.,

$$
l_{2}\left(a^{\prime}\right)=\mu^{\prime} p-e=\left(\mu^{\prime}-e\right) p+(p-1) e
$$

for some $\mu^{\prime} \geq 1$. Equation (35) for $k:=\mu^{\prime}-e$ then reads

$$
0 \equiv a_{k p+e(p-1)}^{\prime}+\sum_{n=p+1}^{2(p-1)} a_{k p+n e}^{\prime} \cdot(-1)^{n-p} \cdot p \cdot H_{n-p}\left(1+\frac{k}{e}\right)
$$

since $i=k p+e n<l_{2}\left(a^{\prime}\right)$ for $n<p-1$ and also $i=k<l_{2}\left(a^{\prime}\right)$, so $a_{i}^{\prime} \equiv 0$ for those $i$. If $\mu^{\prime}<\mu$ we have for $p+1 \leq n \leq 2(p-1)$

$$
k p+n e<(\mu-e) p+2(p-1) e=l\left(a^{\prime}\right)
$$

and hence $p \mid a_{k p+n e}^{\prime}$. So if $\mu^{\prime}<\mu$ then $a_{k p+e(p-1)}^{\prime}$ is the only nonzero term in (42) and we arrive at a contradiction. Therefore $\mu^{\prime} \geq \mu$ and we have found our $a$, or otherwise we arrive at an $a$ with $l_{2}(a)=l(a)$. In either case this proves part (a).

Equation (42) for $k:=\mu-e$ gives

$$
\begin{aligned}
0 & \equiv a_{k p+e(p-1)}+a_{l(a)} \cdot(-1) \cdot p \cdot H_{p-2}\left(1+\frac{\mu-e}{e}\right) \\
& \equiv a_{k p+e(p-1)}-a_{l(a)} \cdot p \cdot \frac{\mu}{e}\left(\bmod p^{2}\right)
\end{aligned}
$$


since $p \mid a_{k p+n e}$ for $k p+n e<k p+2(p-1) e=l(a)$. Note also

$$
H_{p-2}=H_{p-1}-\frac{1}{p-1} \equiv 0-(-1)=1(\bmod p) .
$$

For part (b) we need to show that $v_{\varpi}\left(a_{i} \varpi^{i}\right) \geq l(a)$ for all $i \in \mathbb{Z}$ (and compute the sum over those $i$ for which there is equality). As in the proof of Corollary 40 for $i>l(a)$ and $l(a)-e(p-1)<i<l(a)$ we obviously have $v_{\varpi}\left(a_{i} \varpi^{i}\right)>l(a)$. By (43) we have

$$
\begin{aligned}
a_{l(a)-e(p-1)} \varpi^{l(a)-e(p-1)}+a_{l(a)} \varpi^{l(a)} & \equiv\left(\frac{p \mu}{\varpi^{e(p-1)} e}+1\right) a_{l(a)} \varpi^{l(a)} \\
& =\left(-\frac{\mu}{e}+1\right) a_{l(a)} \varpi^{l(a)}+O\left(\varpi^{l(a)+1}\right)
\end{aligned}
$$

since

$$
\varpi^{e(p-1)}=\left(\zeta_{p}-1\right)^{p-1} \equiv-p\left(\bmod \left(\zeta_{p}-1\right)^{p}\right) .
$$

So if $p \nmid-(\mu / e)+1$ this is the leading term of valuation $l(a)$. For

$$
l(a)-2 e(p-1)<i<l(a)-e(p-1),
$$

since $l_{2}(a) \geq l(a)-e(p-1)$ by part (a), we have $p^{2} \mid a_{i}$ and hence $v_{\varpi}\left(a_{i} \varpi^{i}\right) \geq$ $2 e(p-1)+i>l(a)$. For

$$
l(a)-3 e(p-1)<i \leq l(a)-2 e(p-1)
$$

we have $p^{3} \mid a_{i}$ by (c) of Proposition 34 and hence $v_{\varpi}\left(a_{i} \varpi^{i}\right) \geq 3 e(p-1)+i>l(a)$. Finally for

$$
i \leq l(a)-3 e(p-1)<-e-e(p-1)=-e p
$$

we have by (41)

$v_{\varpi}\left(a_{i} \varpi^{i}\right) \geq-(p-1) i-p e>(p-1) p e-p e=(p-2) p e \geq(2 p-3) e>l(a)$

using the assumption on $l(a)$.

6.2. Isotypic components. We introduce some notation for isotypic components. Recall that

$$
G \cong \Sigma \ltimes \Delta
$$

with $\Sigma$ cyclic of order $f$ and $\Delta$ cyclic of order $e(p-1)$. For any $\Sigma$-orbit $[\eta]$ we define the idempotent

$$
e_{[\eta]}=\sum_{\eta^{\prime} \in \widehat{\Sigma}_{\eta}} e_{\chi} \in \mathbb{Z}_{p}[G]
$$


where the irreducible characters $\chi=\left([\eta], \eta^{\prime}\right)$ of $G$ are parametrized as in Section 3. For any $\mathbb{Z}_{p}[G]$-module $M$ its $[\eta]$-isotypic component

$$
M^{[\eta]}:=e_{[\eta]} M
$$

is a again a $\mathbb{Z}_{p}[G]$-module. The $\Sigma$-orbit

$$
[\eta]=\left\{\eta, \eta^{p}, \eta^{p^{2}}, \ldots, \eta^{p^{f_{\eta}-1}}\right\}=\left\{\eta_{0}^{n_{1}}, \ldots, \eta_{0}^{n_{f_{\eta}}}\right\}
$$

corresponds to an orbit $\left\{n_{1}, \ldots, n_{f_{n}}\right\} \subseteq \mathbb{Z} / e(p-1) \mathbb{Z}$ of residue classes modulo $e(p-1)$ under the multiplication-by- $p$ map, i.e., we have $n_{i+1} \cong n_{i} p \bmod e(p-1)$ where we view the index $i$ as a class in $\mathbb{Z} / f_{\eta} \mathbb{Z}$. We shall use the notation

$$
[\eta]=\left\{n_{1}, \ldots, n_{f_{\eta}}\right\}=\left[n_{i}\right]
$$

to denote both the orbit of residue classes in $\mathbb{Z} / e(p-1) \mathbb{Z}$ and the orbit of characters. By (21) the group

$$
\Delta_{e}:=\operatorname{Gal}\left(K / F\left(\zeta_{p}\right)\right)
$$

acts on $\sqrt[e]{\zeta_{p}-1}=\left.\varphi^{-1}\left(\pi_{K}\right)\right|_{t=0}$ via the character $\eta_{0}$ defined in Section 3 and acts on $\pi_{K}$ via $\eta_{0}^{p}$. The $[\eta]=\left\{n_{1}, \ldots, n_{f_{\eta}}\right\}$-isotypic component of the $\mathbb{Z}_{p}\left[\Sigma \ltimes \Delta_{e}\right]$-module $A_{K}$ is

$$
\left\{a=\sum a_{n} \pi_{K}^{n} \mid a_{n}=0 \quad \text { for } n \bmod e \notin\left\{n_{1}, \ldots, n_{f_{\eta}}\right\}\right\},
$$

but $A_{K}^{[\eta]}$ is much harder to describe since $\pi_{K}$ is not an eigenvector for the full group $\Delta$. However, there is the following fact about leading terms.

Lemma 42. Fix $v \geq 1, a=\sum_{j} a_{j} \pi_{K}^{j} \in A_{K}$ and denote by $e_{\eta} \in \mathcal{O}_{F}[\Delta]$ the idempotent for $\eta=\eta_{0}^{n}$. If

$$
p \cdot l_{v}(a) \equiv n \quad \bmod e(p-1)
$$

then

$$
l_{v}\left(e_{\eta} a\right)=l_{v}(a)
$$

and the leading coefficients modulo $p^{v}$ of $e_{\eta} a$ and a agree. If $a=e_{\eta} a$ is an eigenvector for $\Delta$ then (46) holds.

Proof. Denote by

$$
\omega: \Delta \rightarrow \operatorname{Gal}\left(F\left(\zeta_{p}\right) / F\right) \rightarrow \mathbb{Z}_{p}^{\times}
$$

the Teichmüller character. For $\delta \in \Delta$ we have

$$
\begin{aligned}
\delta\left(\pi_{K}\right) & =\left((1+\pi)^{\omega(\delta)}-1\right)^{1 / e}=\left(\sum_{i=1}^{\infty}\left(\begin{array}{c}
\omega(\delta) \\
i
\end{array}\right) \pi^{i}\right)^{\frac{1}{e}} \\
& =\lambda(\delta) \pi_{K}\left(1+\sum_{i=2}^{\infty} \frac{1}{\omega(\delta)}\left(\begin{array}{c}
\omega(\delta) \\
i
\end{array}\right) \pi^{i-1}\right)^{\frac{1}{e}}
\end{aligned}
$$


where $\lambda(\delta) \in \mu_{e(p-1)}$ satisfies $\lambda(\delta)^{e}=\omega(\delta)$ and $(1+Z)^{1 / e}$ denotes the usual binomial series. Applying $\left.\varphi^{-1}\right|_{t=0}$ we find

$$
\delta\left(\sqrt[e]{\zeta_{p}-1}\right) \equiv \lambda(\delta)^{1 / p} \cdot \sqrt[e]{\zeta_{p}-1} \bmod \varpi^{2}
$$

and since $\sqrt[e]{\zeta_{p}-1} \equiv \sqrt[e(p-1)]{-p} \bmod \varpi^{2}$ we obtain $\lambda(\delta)=\eta_{0}(\delta)^{p}$. In particular, for any $a \in A_{K}$

$$
\delta(a) \equiv \eta_{0}(\delta)^{p \cdot l_{v}(a)} \cdot a_{l_{v}(a)} \cdot \pi_{K}^{l_{v}(a)} \bmod \left(p^{\nu}, \pi_{K}^{l_{v}(a)+1}\right)
$$

and

$$
\begin{aligned}
e_{\eta} a=\frac{1}{e(p-1)} \sum_{\delta \in \Delta} \eta^{-1}(\delta) \delta(a) & \equiv \frac{1}{e(p-1)} \sum_{\delta \in \Delta} \eta_{0}(\delta)^{p \cdot l_{v}(a)-n} \cdot a_{l_{v}(a)} \cdot \pi_{K}^{l_{v}(a)} \\
& \equiv \begin{cases}a_{l_{v}(a)} \cdot \pi_{K}^{l_{v}(a)} & \text { if } p \cdot l_{v}(a) \equiv n \bmod e(p-1), \\
0 & \text { if } p \cdot l_{v}(a) \neq \equiv \bmod e(p-1),\end{cases}
\end{aligned}
$$

where the congruences are modulo $\left(p^{v}, \pi_{K}^{l_{v}(a)+1}\right)$. This implies both statements in the lemma.

Remark 43. With the notation introduced in this section we have

$$
e_{[\eta]}=\sum_{i=1}^{f_{\eta}} e_{\eta^{p^{i}}} .
$$

6.3. The main result. We view $\Sigma$ as a subgroup of $G$ such that $\sqrt[e(p-1)]{-p} \in K^{\Sigma}$, where $\sqrt[e(p-1)]{-p}$ is the choice of root corresponding to our choice of root $\pi_{K}$ of $\pi$. Then the $\mathbb{Z}_{p}[\Sigma]$-algebra $\mathbb{Z}_{p}[G]$ is finite free of rank $e(p-1)$. For each choice of $\eta$ the $[\eta]$-isotypic component of $\mathbb{Z}_{p}[G]$ is free of rank $f_{\eta}$ over $\mathbb{Z}_{p}[\Sigma]$ and for each $\eta \neq \omega$ the $[\eta]$-isotypic component

$$
\left(A_{K}^{\psi=1}(1)\right)^{[\eta]}
$$

of $A_{K}^{\psi=1}(1)$ is free of rank $f_{\eta}$ over $\mathbb{Z}_{p}[\Sigma] \llbracket \gamma_{1}-1 \rrbracket$. Write

$$
[\eta]=\left\{n_{1}, \ldots, n_{f_{\eta}}\right\}=\left[n_{1}\right] \subseteq \mathbb{Z} / e(p-1) \mathbb{Z}
$$

and pick representatives $n_{i} \in \mathbb{Z}$ with

$$
0<n_{i}<e(p-1), \quad i=1, \ldots, f_{\eta} .
$$

Note that our running assumption $\left.\eta\right|_{\Delta_{e}} \neq 1$ implies $e \nmid n_{i}$.

Proposition 44. Fix $\left.\eta\right|_{\Delta_{e}} \neq 1$ and let $\left\{\alpha_{i} \mid i=1, \ldots, f_{\eta}\right\}$ be $a \mathbb{Z}_{p}[\Sigma] \llbracket\left[\gamma_{1}-1 \rrbracket\right.$-basis of $\left(A_{K}^{\psi=1}(1)\right)^{[\eta]}$. Let $n_{i, r}$ be representatives for the residue classes

$$
\left[n_{1}-r e\right] \subseteq \mathbb{Z} / e(p-1) \mathbb{Z}
$$


with

$$
0<n_{i, r}<e(p-1)
$$

indexed such that $n_{i}-r e \equiv n_{i, r} \bmod e(p-1)$. Consider the two $\mathbb{Z}_{p}[\Sigma]$-lattices

and

$$
L_{r}:=\bigoplus_{i=1}^{f_{\eta}} \mathbb{Z}_{p}[\Sigma] \cdot\left(\nabla^{r-1} \alpha_{i}^{\sigma^{-1}}\right)\left(\sqrt[e]{\zeta_{p}-1}\right)
$$

$$
\mathcal{O}_{K}^{\left[n_{1}-r e\right]}=\bigoplus_{i=1}^{f_{\eta}} \mathcal{O}_{F} \cdot(\sqrt[e(p-1)]{-p})^{n_{i, r}}
$$

in the $\left[n_{1}-r e\right]$-isotypic component

$$
K^{\left[n_{1}-r e\right]}=\bigoplus_{i=1}^{f_{\eta}} F \cdot(\sqrt[e(p-1)]{-p})^{n_{i, r}}=\bigoplus_{i=1}^{f_{\eta}} F \cdot(\sqrt[e(p-1)]{-p})^{n_{i}-r e}
$$

of $K$. Then the conjunction of (16) (in Proposition 7) for $\chi=\left(\left[n_{1}-r e\right], \eta^{\prime}\right)$ over all $\eta^{\prime}$ holds if and only if $L_{r}$ and $\mathcal{O}_{K}^{\left[n_{1}-r e\right]}$ have the same $\mathbb{Z}_{p}[\Sigma]$-volume, i.e.,

$$
\operatorname{Det}_{\mathbb{Z}_{p}[\Sigma]} L_{r}=\operatorname{Det}_{\mathbb{Z}_{p}[\Sigma]} \mathcal{O}_{K}^{\left[n_{1}-r e\right]}
$$

inside $\operatorname{Det}_{\mathbb{Q}_{p}[\Sigma]} K^{\left[n_{1}-r e\right]}$.

Proof. Let $\alpha$ be a $\Lambda_{K} e_{\left[n_{1}\right]}$-basis of $\left(A_{K}^{\psi=1}(1)\right)^{\left[n_{1}\right]}$. Then

$$
\beta_{I w}:=\left(\operatorname{Exp}_{\mathbb{Z}_{p}}^{*}\right)^{-1}(\alpha)
$$

is a $\Lambda_{K} e_{\left[n_{1}\right]}$-basis of $H_{I w}^{1}\left(K, \mathbb{Z}_{p}(1)\right)^{\left[n_{1}\right]}$ and the element

$$
\beta=\operatorname{pr}_{1,1-r}\left(\beta_{I w}\right)
$$

of Corollary 12 is a $\mathbb{Z}_{p}[G] e_{\left[n_{1}-r e\right]}$-basis of $\left(H^{1}\left(K, \mathbb{Z}_{p}(1-r)\right) / \text { tor }\right)^{\left[n_{1}-r e\right]}$. This follows from the fact that the isomorphism $\mathrm{pr}_{1,1-r}$ of Lemma 8 is not $\Lambda_{K}$-linear but $\Lambda_{K}-\kappa_{-r}$-semilinear, where $\kappa_{j}$ is the automorphism of $\Lambda_{K}$ given by $g \mapsto g \chi^{\text {cyclo }}(g)^{j}$ for $g \in G \times \Gamma_{K}$. Theorem 9 and Proposition 13 imply

$$
\begin{aligned}
\exp _{\mathbb{Q}_{p}(r)}^{*}(\beta) & =\left.\frac{1}{(r-1) !}\left(\frac{d}{d t}\right)^{r-1} p^{-1} \varphi^{-1}(\alpha)\right|_{t=0} \\
& =\frac{p^{-r}}{(r-1) !}\left(\nabla^{r-1} \alpha^{\sigma^{-1}}\right)\left(\sqrt[e]{\zeta_{p}-1}\right)
\end{aligned}
$$

Hence the $\mathbb{Z}_{p}[G] e_{\left[n_{1}-r e\right]}$-lattice

$$
\mathbb{Z}_{p}[G] \cdot(r-1) ! \cdot p^{r-1} \cdot \exp _{\mathbb{Q}_{p}(r)}^{*}(\beta) \subset K^{\left[n_{1}-r e\right]}
$$

is free over $\mathbb{Z}_{p}[\Sigma]$ with basis

$$
(r-1) ! \cdot p^{r-1} \cdot \frac{p^{-r}}{(r-1) !}\left(\nabla^{r-1} \alpha_{i}^{\sigma^{-1}}\right) \sqrt[e]{\zeta_{p}-1}=p^{-1} \cdot\left(\nabla^{r-1} \alpha_{i}^{\sigma^{-1}}\right)\left(\sqrt[e]{\zeta_{p}-1}\right)
$$


where $i=1, \ldots, f_{\eta}$. Now the conjunction of (16) for $\chi=\left(\left[n_{1}-r e\right], \eta^{\prime}\right)$ over all $\eta^{\prime}$ is equivalent to the statement that the lattice (48) and the $\left[n_{1}-r e\right]$-isotypic component of the inverse different

$$
\left(\sqrt[e]{\zeta_{p}-1}\right)^{-(e(p-1)-1)} \mathcal{O}_{K}
$$

have the same $\mathbb{Z}_{p}[\Sigma]$-volume. Since $e \nmid n_{1}$ we have

$$
\left(\left(\sqrt[e]{\zeta_{p}-1}\right)^{-(e(p-1)-1)} \mathcal{O}_{K}\right)^{\left[n_{1}-r e\right]}=\left(p^{-1} \mathcal{O}_{K}\right)^{\left[n_{1}-r e\right]}
$$

and the statement follows.

6.4. Prooffor $r=1,2$ and small $e$. We retain the notation of the previous section. As in Proposition 24 denote by $\xi$ a $\mathbb{Z}_{p}[\Sigma]$-basis of $\mathcal{O}_{F}$.

Proposition 45. There exists $a \mathbb{Z}_{p}[\Sigma] \llbracket \gamma_{1}-1 \rrbracket$-basis

$$
\alpha_{i}=\xi \cdot \pi_{K}^{l\left(\alpha_{i}\right)}+\cdots \in A_{K}^{\psi=1}, \quad i=1, \ldots, f_{\eta}
$$

of $\left(A_{K}^{\psi=1}\right)^{\left[n_{1}-e\right]}$ with

$$
l\left(\alpha_{i}\right)= \begin{cases}n_{i}-e & \text { if } p \nmid n_{i}, \\ n_{i}-e+e(p-1) & \text { if } p \mid n_{i} .\end{cases}
$$

Proof. By Nakayama's lemma it suffices to find a $\mathbb{F}_{p}[\Sigma]$-basis for

$$
\left(A_{K}^{\psi=1}\right)^{\left[n_{1}-e\right]} /\left(p, \gamma_{1}-1\right) \cong\left(A_{K}^{\psi=1} /\left(p, \gamma_{1}-1\right)\right)^{\left[n_{1}-e\right]} .
$$

By Lemma 32 we have $A_{K}^{\psi=1} / p A_{K}^{\psi=1}=E_{K}^{\psi=1}$. By Proposition 38 (reductions mod $p$ of) elements $\alpha_{i}$ as described in Proposition 45 exist in $E_{K}^{\psi=1}$. By projection and Lemma 42 we can also assume that they are in the $\left[n_{1}-e\right]$-isotypic component. Let $a^{\prime}$ be a nonzero $\mathbb{Z}_{p}[\Sigma]$-linear combination of the $\alpha_{i}$ and assume

$$
a^{\prime} \equiv\left(\gamma_{1}-1\right) a \bmod p
$$

for some $a \in A_{K}^{\psi=1}$. By Lemma 46 below we have $l\left(a^{\prime}\right) \geq-e+e(p-1)$. Since $l\left(a^{\prime}\right)=l\left(\alpha_{i}\right)$ for some $i$, this implies

$$
l\left(a^{\prime}\right) \equiv-e+e(p-1) \equiv-2 e \bmod p .
$$

Using Lemma 46 again we have $l(a) \leq l\left(a^{\prime}\right)-e(p-1) \equiv-e \bmod p$. Since $l(a) \not \equiv-e \bmod p$ by Proposition 38 we have strict inequality. Lemma 46 then shows $p \mid l(a)$ and hence $p \mid l\left(a^{\prime}\right)$, contradicting $l\left(a^{\prime}\right) \equiv-2 e \bmod p$. We conclude that the $\alpha_{i}$ are linearly independent in (49). Since the $\mathbb{F}_{p}[\Sigma]$-rank of (49) is $f_{\eta}$ this finishes the proof. 
Lemma 46. For $a \in E_{K}^{\psi=1}$ with $l(a)=j p^{\kappa}$ with $p \nmid j$ we have

$$
l\left(\left(\gamma_{1}-1\right) a\right)=(j+e(p-1)) p^{\kappa} .
$$

In particular

$$
l\left(\left(\gamma_{1}-1\right) a\right) \geq l(a)+e(p-1)
$$

with equality if and only if $p \nmid l(a)$, and

$$
l\left(\left(\gamma_{1}-1\right) a\right) \geq-e+e(p-1)
$$

for all $a \in E_{K}^{\psi=1}$.

Proof. Since $\chi^{\text {cyclo }}\left(\gamma_{1}\right)=1+p$ we find from (20) that (in $E_{K}$ )

$$
\gamma_{1}(\pi)=\pi+\pi^{p}+\pi^{p+1}
$$

and hence for $n=j p^{\kappa}$

$$
\begin{aligned}
\left(\gamma_{1}-1\right) \pi_{K}^{n} & =\left(\pi+\pi^{p}+\pi^{p+1}\right)^{n / e}-\pi^{n / e}=\pi_{K}^{n}\left(\left(1+\pi^{p-1}+\pi^{p}\right)^{n / e}-1\right) \\
& =\pi_{K}^{n}\left(\left(1+\pi^{p^{\kappa}(p-1)}+\pi^{p^{k+1}}\right)^{j / e}-1\right) \\
& =\frac{j}{e} \cdot \pi_{K}^{n+e p^{\kappa}(p-1)}+\cdots
\end{aligned}
$$

and this is indeed the leading term since $p \nmid j$. The last assertion follows from Proposition 34(a).

Proposition 47. If $e<p$, the identity (47) holds for $r=1$.

Proof. We first remark that for each $i$ we have

$$
v_{\varpi}\left(\alpha_{i}\right)=l\left(\alpha_{i}\right)= \begin{cases}n_{i}-e & \text { if } p \nmid n_{i}, \\ n_{i}-e+e(p-1) & \text { if } p \mid n_{i}\end{cases}
$$

by Corollary 40 and Proposition 41 . Note that there is at most one $n_{i}, n_{1}$ say, with

$$
0<n_{1} \leq e-1
$$

since all the $n_{i}$ lie in the same residue class modulo $p-1$ and $e \leq p-1$. Then

$$
n_{2}=p n_{1} \leq e p-p<e p-e=e(p-1)
$$

and conversely, $p \mid n_{2}$ if and only if $0<n_{1}:=n_{2} / p \leq e-1$. For all other $i$ we have $n_{i}-e=n_{i, 1}$. So if no $n_{i}-e$ is negative then

$$
q_{i}:=\alpha_{i}^{\sigma^{-1}}\left(\sqrt[e]{\zeta_{p}-1}\right) \in K
$$

is already a basis of $\mathcal{O}_{K}^{\left[n_{1}-e\right]}$. Otherwise

$$
p \cdot q_{1}, p^{-1} \cdot q_{2}, q_{3}, \ldots, q_{f_{\eta}}
$$


is a basis of $\mathcal{O}_{K}^{\left[n_{1}-e\right]}$. Since $L_{1}$ is the span of the $q_{i}$ the statement follows.

Remark 48. Although not covered by Proposition 2, it is in fact true that the equivariant local Tamagawa number conjecture for $r=1$ is equivalent to (47) for $r=1$ and so Proposition 47 proves this conjecture for $e<p$. However, for $r=1$ one can give a direct proof without any assumption on $e$ other than $p \nmid e$ by studying the exponential map instead of the dual exponential map. Since the exponential power series gives a $G$-equivariant isomorphism

$$
\exp : p \cdot \mathcal{O}_{K} \cong 1+p \cdot \mathcal{O}_{K},
$$

the (equivariant) relative volume of $\exp \left(\mathcal{O}_{K}\right)$ and $\left(\mathcal{O}_{K}^{\times}\right)^{\wedge} \subseteq H^{1}\left(K, \mathbb{Z}_{p}(1)\right)$ can be easily computed. For more work on the case $r=1$, see [Bley and Cobbe 2016] and references therein.

To prepare for the proof of Proposition 51 below we need to compute $v_{\varpi}\left(\nabla \alpha_{i}\right)$, i.e., prove the analogues of Corollary 40 and Proposition 41 for $\nabla a \in A_{K}^{\psi=p}$.

Lemma 49. Assume $e<p / 2$. For $a \in A_{K}^{\psi=1}$ with

$$
p \nmid l(a)<-e+e(p-1)
$$

or with

$$
l(a)=\mu p-e+e(p-1)
$$

and chosen as in Proposition 41(a) we have

$$
v_{\varpi}(\nabla a)=l(\nabla a)=l(a)-e .
$$

Proof. Since

$$
\nabla \pi_{K}^{j}=\frac{j}{e} \pi_{K}^{j-e}+\frac{j}{e} \pi_{K}^{j}
$$

it is clear that $l(\nabla a)=l(a)-e$ if $p \nmid l(a)$. To compute $v_{\varpi}(\nabla a)$, note that from the proof of Corollary 40 we already know

$$
v_{\varpi}\left(a_{j} \varpi^{j}\right)>l(a)
$$

for $j \neq l(a)$. But this implies

$$
v_{\varpi}\left(a_{j} \frac{j}{e} \varpi^{j-e}\right)>l(a)-e, \quad v_{\varpi}\left(a_{j} \frac{j}{e} \varpi^{j}\right)>l(a)>l(a)-e
$$

for $j \neq l(a)$. This finishes the proof for the case $p \nmid l(a)<-e+e(p-1)$. If

$$
l(a)=\mu p-e+e(p-1)
$$

then recall from the proof of Proposition 41(b) that we had to compute modulo $p^{2}$ and there were two terms in (44) with valuation $l(a)$ arising from $j=l(a)$ and 
$j=l(a)-e(p-1)$. Normalizing the leading coefficient to be $\xi$ (as in the $\left.\alpha_{i}\right)$ we have

$$
a \equiv \xi \cdot \frac{\mu p}{e} \cdot \pi_{K}^{l(a)-e(p-1)}+\cdots+\xi \cdot \pi_{K}^{l(a)}+\cdots \bmod p^{2}
$$

and

$$
\nabla a \equiv \xi \cdot \frac{\mu p}{e} \cdot \frac{\mu p-e}{e} \cdot \pi_{K}^{l(a)-e-e(p-1)}+\cdots+\xi \cdot \frac{l(a)}{e} \cdot \pi_{K}^{l(a)-e}+\cdots \bmod p^{2}
$$

and hence

$$
\begin{aligned}
\frac{\mu p}{e} \cdot \frac{\mu p-e}{e} \cdot \varpi^{l(a)-e-e(p-1)}+\frac{l(a)}{e} & \cdot \varpi^{l(a)-e} \\
& \equiv\left(-\frac{\mu}{e} \cdot \frac{\mu p-e}{e}+\frac{l(a)}{e}\right) \cdot \varpi^{l(a)-e} \bmod p^{2} .
\end{aligned}
$$

Computing the leading coefficient modulo $p$ we find

$$
\left(\frac{\mu}{e}+\frac{-2 e}{e}\right)=\frac{\mu}{e}-2,
$$

which is divisible by $p$ if and only if $p \mid \mu-2 e$. Since $e<p / 2$ we have

$$
-p<-2 e<\mu-2 e<\frac{e(p-1)}{p}-2 e=\left(-1-\frac{1}{p}\right) e<0
$$

and hence $p \nmid \mu-2 e$. In the proof of Proposition 41(b) we showed $v_{\varpi}\left(a_{j} \varpi^{j}\right)>l(a)$ for $j \neq l(a), l(a)-e(p-1)$ and as above this implies that the corresponding terms in $\nabla a$ all have valuation larger than $l(a)-e$.

We handle the case $p \mid l(a)$ in a separate lemma. Similar to Proposition 41 we need to compute modulo $p^{2}$.

Lemma 50. Assume $e<p / 4$ and $0<\mu p<-e+e(p-1)$. Then there exists $a \in\left(A_{K}^{\psi=1}\right)^{[\mu p]}$ with $l(a)=\mu p$ and

$$
v_{\varpi}(\nabla a)=l(\nabla a)=\mu p-e+e(p-1) .
$$

Moreover we can choose $a$ with any leading coefficient.

Proof. The statement about the leading coefficient will be clear from the proof, so to alleviate notation we take the leading coefficient to be 1 . First we can find $a^{\prime} \in A_{K}^{\psi=1}$ with

$$
a^{\prime} \equiv \pi_{K}^{\mu p}-\pi_{K}^{\mu p+e(p-1)}+\cdots\left(\bmod p^{2}\right),
$$

i.e., with $a_{i}^{\prime} \equiv 0$ for all $i<\mu p+e(p-1)$ and $i \neq \mu p$. To see this, first note that (35) is satisfied for $k=\mu$ since $H_{p-1} \equiv 0(\bmod p)$ (and we take $a_{\mu p+n e}^{\prime}$ arbitrary but divisible by $p$ for $n=p+1, \ldots, 2(p-1)$ ). In any Equation (35) with index $k<\mu$ the coefficient $a_{\mu p}^{\prime}$ does not occur on the left-hand side since $k p+n e$ is a multiple of $p$ only for $n=0$ among $n \in\{0, \ldots, p-1, p+1, \ldots, 2(p-1)\}$. On the right-hand 
side we always have $a_{k}^{\prime} \equiv 0$ since $k<\mu<\mu p$. Similarly, the coefficient $a_{\mu p+e(p-1)}^{\prime}$ does not occur on the left-hand side for $k<\mu$ since $k p+n e=\mu p+e(p-1)$ implies $n \equiv-1(\bmod p)$, i.e., $n=p-1$. So the fact that $a_{i}^{\prime} \neq \equiv 0$ for $i=\mu p, \mu p+e(p-1)$ forces no further nonzero terms in equations with index $k<\mu$. Equations (35) with index $k>\mu$ can always be satisfied inductively by adjusting the variable $a_{k p+(p-1) e}^{\prime}$ since $a_{k p+(p-1) e}^{\prime}$ does not occur in any equation with index $k^{\prime}<k$.

With the notation introduced in Section 6.2 set

$$
a=e_{[\mu p]} a^{\prime} \in\left(A_{K}^{\psi=1}\right)^{[\mu p]}
$$

so that $l(a)=l_{2}(a)=\mu p$ by Lemma 42 . We have

$$
\nabla a^{\prime} \equiv \frac{\mu p}{e} \cdot \pi_{K}^{\mu p-e}+\frac{\mu p}{e} \cdot \pi_{K}^{\mu p}-\frac{\mu p+e(p-1)}{e} \cdot \pi_{K}^{\mu p-e+e(p-1)}+\cdots\left(\bmod p^{2}\right)
$$

and hence

$$
\begin{aligned}
\nabla a & =\nabla e_{[\mu p]} a^{\prime}=e_{[\mu p-e]} \nabla a^{\prime} \\
& \equiv \frac{\mu p}{e} \cdot \pi_{K}^{\mu p-e}+\cdots-\left(\frac{\mu p+e(p-1)}{e}-\frac{\mu p}{e} x\right) \cdot \pi_{K}^{\mu p-e+e(p-1)}+\cdots\left(\bmod p^{2}\right),
\end{aligned}
$$

where $x$ is the coefficient of $\pi_{K}^{\mu p-e+e(p-1)}$ in the expansion of $e_{[\mu p-e]}\left(\pi_{K}^{\mu p-e}+\pi_{K}^{\mu p}\right)$. Moreover

$$
l(\nabla a)=l\left(\nabla e_{[\mu p]} a^{\prime}\right)=l\left(e_{[\mu p-e]} \nabla a^{\prime}\right)=l\left(\nabla a^{\prime}\right)=\mu p-e+e(p-1) .
$$

In order to show that $v_{\varpi}(\nabla a)=l(\nabla a)$ write

$$
\nabla a=\sum_{i} b_{i} \cdot \pi_{K}^{i}
$$

The terms for $i=\mu p-e$ and $i=\mu p-e+e(p-1)$ contribute the leading term in the variable $\varpi$

$$
\begin{aligned}
\frac{\mu p}{e} \cdot \varpi^{\mu p-e}-\left(\frac{\mu p+e(p-1)}{e}-\frac{\mu p}{e} x\right) \cdot \varpi^{\mu p-e+e(p-1)}+\cdots \\
=\left(-\frac{\mu}{e}-\frac{\mu p+e(p-1)}{e}+\frac{\mu p}{e} x\right) \cdot \varpi^{\mu p-e+e(p-1)}+\cdots
\end{aligned}
$$

since, similarly to (44), we have $p \nmid-\frac{\mu}{e}+1$ as $e<p$. For the terms with $i \neq$ $\mu p-e+e(p-1), \mu p-e$ we must again verify that

$$
v_{\varpi}\left(b_{i} \varpi^{i}\right)>\mu p-e+e(p-1) .
$$

This is clear for $i>\mu p-e+e(p-1)$ and for

$$
\mu p-e<i<\mu p-e+e(p-1)
$$


since then $p \mid b_{i}$. For $i<\mu p-e$ it suffices to show by (51) that we have instead

$$
v_{\varpi}\left(a_{i} \varpi^{i}\right)>\mu p+e(p-1)
$$

for $i<\mu p$. Since $l_{2}(a)=\mu p$ we have $v_{\varpi}\left(a_{i}\right) \geq 2 e(p-1)$ for

$$
\mu p-e(p-1)<i<\mu p
$$

and hence $v_{\varpi}\left(a_{i} \varpi^{i}\right)>\mu p+e(p-1)$. For

$$
\mu p-2 e(p-1)<i \leq \mu p-e(p-1)
$$

we have by $v_{\varpi}\left(a_{i}\right) \geq 3 e(p-1)$ by Proposition 34(a) since

$$
i \leq \mu p-e(p-1)<-\left(3-\frac{2}{p}\right) \cdot e .
$$

Indeed this last inequality is equivalent to

$$
\mu p<\left((p-1)-\left(3-\frac{2}{p}\right)\right) \cdot e \Longleftrightarrow \mu<e-\left(\frac{4}{p}-\frac{2}{p^{2}}\right) \cdot e,
$$

which holds by our assumption $4 e<p$, noting that $e-1$ is the maximal value for $\mu$. Finally for

$$
i \leq \mu p-2 e(p-1)<-e-e(p-1)=-e p
$$

we have by (41)

$$
\begin{aligned}
v_{\varpi}\left(a_{i} \varpi^{i}\right) & \geq-(p-1) i-p e>(p-1) p e-p e=(p-2) p e \\
& \geq(2 p-3) e=-e+2 e(p-1)>\mu p+e(p-1) .
\end{aligned}
$$

Proposition 51. If $e<p / 4$ the identity (47) holds for $r=2$.

Proof. By Lemmas 49 and 50 we can choose $\alpha_{i}$ such that

$$
v_{\varpi}\left(\nabla \alpha_{i}\right)=l\left(\nabla \alpha_{i}\right)= \begin{cases}n_{i}-2 e & \text { if } p \nmid n_{i} \text { and } p \nmid n_{i}-e, \\ n_{i}-2 e+e(p-1) & \text { if } p \mid n_{i} \text { or } p \mid n_{i}-e .\end{cases}
$$

As in the proof of Proposition 47, for each $0<n_{1}<e$ there is a unique $n_{2}=p n_{1}$ divisible by $p$. Similarly for each $n_{h}$ with $e<n_{h}<2 e$ (which is unique if it exists) there is a unique

$$
n_{h+1}-e=p\left(n_{h}-e\right)
$$

divisible by $p$. Note here that $n_{h} \leq 2 e-1$ and hence

$$
n_{h+1} \leq p(e-1)+e<e(p-1)
$$

using $2 e<p$. Let

$$
q_{i}:=\nabla \alpha_{i}^{\sigma^{-1}}\left(\sqrt[e]{\zeta_{p}-1}\right) \in K
$$


be the basis of $L_{2}$. We again find that

$$
\begin{aligned}
p \cdot q_{1}, p^{-1} \cdot q_{2}, \ldots, p \cdot q_{h}, p^{-1} \cdot q_{h+1}, \ldots, q_{f_{\eta}} & \text { if } n_{1}<e \text { and } e<n_{h}<2 e, \\
p \cdot q_{1}, p^{-1} \cdot q_{2}, \ldots, q_{h}, q_{h+1}, \ldots, q_{f_{\eta}} & \text { if } n_{1}<e \text { and } \nexists e<n_{h}<2 e, \\
q_{1}, q_{2}, \ldots, p \cdot q_{h}, p^{-1} \cdot q_{h+1}, \ldots, q_{f_{\eta}} & \text { if } \nexists n_{1}<e \text { and } e<n_{h}<2 e, \\
q_{1}, q_{2}, \ldots, q_{h}, q_{h+1}, \ldots, q_{f_{\eta}} & \text { if } \nexists n_{1}<e \text { nor } e<n_{h}<2 e
\end{aligned}
$$

is a basis of $\mathcal{O}_{K}^{\left[n_{1}-2 e\right]}$ and the statement follows.

\section{Acknowledgements}

We would like to thank the referee for a very careful reading of the manuscript, which helped to improve our exposition a lot.

\section{References}

[Benois and Berger 2008] D. Benois and L. Berger, "Théorie d'Iwasawa des représentations cristallines, II”, Comment. Math. Helv. 83:3 (2008), 603-677. MR 2410782 Zbl 1157.11041

[Bley and Cobbe 2016] W. Bley and A. Cobbe, "The equivariant local $\epsilon$-constant conjecture for unramified twists of $\mathbb{Z}_{p}(1)$ ", preprint, 2016. arXiv 1602.07858

[Bloch and Kato 1990] S. Bloch and K. Kato, " $L$-functions and Tamagawa numbers of motives", pp. 333-400 in The Grothendieck Festschrift, I, edited by P. Cartier et al., Progr. Math. 86, Birkhäuser, Boston, 1990. MR 1086888 Zbl 0768.14001

[Breuning 2004] M. Breuning, "Equivariant local epsilon constants and étale cohomology", J. London Math. Soc. (2) 70:2 (2004), 289-306. MR 2078894 Zbl 1068.11075

[Burns and Flach 2006] D. Burns and M. Flach, "On the equivariant Tamagawa number conjecture for Tate motives, II”, Doc. Math. Extra Vol. (2006), 133-163. MR 2290586 Zbl 1156.11042

[Cherbonnier and Colmez 1999] F. Cherbonnier and P. Colmez, "Théorie d'Iwasawa des représentations $p$-adiques d'un corps local”, J. Amer. Math. Soc. 12:1 (1999), 241-268. MR 1626273 Zbl 0933.11056

[Coleman 1979] R. F. Coleman, "Division values in local fields", Invent. Math. 53:2 (1979), 91-116. MR 560409 Zbl 0429.12010

[Colmez 1999] P. Colmez, "Représentations cristallines et représentations de hauteur finie", J. Reine Angew. Math. 514 (1999), 119-143. MR 1711279 Zbl 1191.11032

[Daigle 2014] J. Daigle, On the local Tamagawa Number Conjecture for Tate motives, Ph.D. thesis, Caltech, 2014, available at http://thesis.library.caltech.edu/8427/.

[Deligne 1973] P. Deligne, "Les constantes des équations fonctionnelles des fonctions $L$ ", pp. 501597 in Modular functions of one variable, II (Antwerp, 1972), edited by P. Deligne and W. Kuijk, Lecture Notes in Math 349, Springer, Berlin, 1973. MR 0349635 Zbl 0271.14011

[Deligne 1987] P. Deligne, "Le déterminant de la cohomologie", pp. 93-177 in Current trends in arithmetical algebraic geometry (Arcata, CA, 1985), edited by K. A. Ribet, Contemp. Math. 67, Amer. Math. Soc., Providence, RI, 1987. MR 902592 Zbl 0629.14008

[Fontaine and Perrin-Riou 1994] J.-M. Fontaine and B. Perrin-Riou, "Autour des conjectures de Bloch et Kato: Cohomologie galoisienne et valeurs de fonctions L", pp. 599-706 in Motives (Seattle, 
WA, 1991), edited by U. Jannsen et al., Proc. Sympos. Pure Math. 55, Amer. Math. Soc., Providence, RI, 1994. MR 1265546 Zbl 0821.14013

[Fröhlich 1976] A. Fröhlich, "Arithmetic and Galois module structure for tame extensions", J. Reine Angew. Math. 286/287 (1976), 380-440. MR 0432595 Zbl 0385.12004

[Fukaya and Kato 2006] T. Fukaya and K. Kato, "A formulation of conjectures on p-adic zeta functions in noncommutative Iwasawa theory", pp. 1-85 in Proceedings of the St. Petersburg Mathematical Society, XII, edited by N. N. Uraltseva, Amer. Math. Soc. Transl. (2) 219, Amer. Math. Soc., Providence, RI, 2006. MR 2276851 Zbl 1238.11105

[Kato 1993] K. Kato, "Lectures on the approach to Iwasawa theory for Hasse-Weil $L$-functions via $B_{\mathrm{dR}}$, II”, unpublished preprint, 1993.

[Lang 2002] S. Lang, Algebra, 3rd ed., Graduate Texts in Mathematics 211, Springer, New York, 2002. MR 1878556 Zbl 0984.00001

[Neukirch 1999] J. Neukirch, Algebraic number theory, Grundlehren der Mathematischen Wissenschaften 322, Springer, Berlin, 1999. MR 1697859 Zbl 0956.11021

[Neukirch et al. 2000] J. Neukirch, A. Schmidt, and K. Wingberg, Cohomology of number fields, Grundlehren der Mathematischen Wissenschaften 323, Springer, Berlin, 2000. MR 1737196 Zbl 0948.11001

[Perrin-Riou 1990] B. Perrin-Riou, "Théorie d'Iwasawa $p$-adique locale et globale", Invent. Math. 99:2 (1990), 247-292. MR 1031902 Zbl 0715.11030

[Perrin-Riou 1994] B. Perrin-Riou, “Théorie d'Iwasawa des représentations $p$-adiques sur un corps local”, Invent. Math. 115:1 (1994), 81-149. MR 1248080 Zbl 0838.11071

[Serre 1979] J.-P. Serre, Local fields, Graduate Texts in Mathematics 67, Springer, New York, 1979. MR 554237 Zbl 0423.12016

[Venjakob 2013] O. Venjakob, “On Kato's local $\epsilon$-isomorphism conjecture for rank-one Iwasawa modules”, Algebra Number Theory 7:10 (2013), 2369-2416. MR 3194646 Zbl 1305.11095

[Washington 1997] L. C. Washington, Introduction to cyclotomic fields, 2nd ed., Graduate Texts in Mathematics 83, Springer, New York, 1997. MR 1421575 Zbl 0966.11047

Communicated by Kiran S. Kedlaya

Received 2015-08-25 Revised 2016-03-09 Accepted 2016-05-18

gdaigle@oxy.edu

Department of Mathematics, Occidental College, 1600 Campus Road, Los Angeles, CA 90041, United States

flach@caltech.edu

Department of Mathematics, Caltech, 253/37,

Pasadena, CA 91125, United States 


\section{Algebra \& Number Theory}

msp.org/ant

\section{EDITORS}

MANAGING EDITOR

Bjorn Poonen

Massachusetts Institute of Technology

Cambridge, USA

\author{
EDITORIAL BOARD CHAIR \\ David Eisenbud \\ University of California \\ Berkeley, USA
}

BOARD OF EDITORS

$\begin{aligned} \text { Dave Benson } & \text { University of Aberdeen, Scotland } & \text { Susan Montgomery } & \text { University of Southern California, USA } \\ \text { Richard E. Borcherds } & \text { University of California, Berkeley, USA } & \text { Shigefumi Mori } & \text { RIMS, Kyoto University, Japan } \\ \text { John H. Coates } & \text { University of Cambridge, UK } & \text { Raman Parimala } & \text { Emory University, USA } \\ \text { J-L. Colliot-Thélène } & \text { CNRS, Université Paris-Sud, France } & \text { Jonathan Pila } & \text { University of Oxford, UK } \\ \text { Brian D. Conrad } & \text { Stanford University, USA } & \text { Anand Pillay } & \text { University of Notre Dame, USA } \\ \text { Hélène Esnault } & \text { Freie Universität Berlin, Germany } & \text { Victor Reiner } & \text { University of Minnesota, USA } \\ \text { Hubert Flenner } & \text { Ruhr-Universität, Germany } & \text { Peter Sarnak } & \text { Princeton University, USA } \\ \text { Sergey Fomin } & \text { University of Michigan, USA } & \text { Joseph H. Silverman } & \text { Brown University, USA } \\ \text { Edward Frenkel } & \text { University of California, Berkeley, USA } & \text { Michael Singer } & \text { North Carolina State University, USA } \\ \text { Andrew Granville } & \text { Université de Montréal, Canada } & \text { Vasudevan Srinivas } & \text { Tata Inst. of Fund. Research, India } \\ \text { Joseph Gubeladze } & \text { San Francisco State University, USA } & \text { J. Toby Stafford } & \text { University of Michigan, USA } \\ \text { Roger Heath-Brown } & \text { Oxford University, UK } & \text { Ravi Vakil } & \text { Stanford University, USA } \\ \text { Craig Huneke } & \text { University of Virginia, USA } & \text { Michel van den Bergh } & \text { Hasselt University, Belgium } \\ \text { Kiran S. Kedlaya } & \text { Univ. of California, San Diego, USA } & \text { Marie-France Vignéras } & \text { Université Paris VII, France } \\ \text { János Kollár } & \text { Princeton University, USA } & \text { Kei-Ichi Watanabe } & \text { Nihon University, Japan } \\ \text { Yuri Manin } & \text { Northwestern University, USA } & \text { Efim Zelmanov } & \text { University of California, San Diego, USA } \\ \text { Philippe Michel } & \text { École Polytechnique Fédérale de Lausanne } & \text { Shou-Wu Zhang } & \text { Princeton University, USA }\end{aligned}$

PRODUCTION

production@msp.org

Silvio Levy, Scientific Editor

See inside back cover or msp.org/ant for submission instructions.

The subscription price for 2016 is US $\$ 290$ /year for the electronic version, and $\$ 485 /$ year (+\$55, if shipping outside the US) for print and electronic. Subscriptions, requests for back issues and changes of subscribers address should be sent to MSP.

Algebra \& Number Theory (ISSN 1944-7833 electronic, 1937-0652 printed) at Mathematical Sciences Publishers, 798 Evans Hall \#3840, c/o University of California, Berkeley, CA 94720-3840 is published continuously online. Periodical rate postage paid at Berkeley, CA 94704, and additional mailing offices.

ANT peer review and production are managed by EditFLow ${ }^{\circledR}$ from MSP.

\section{PUBLISHED BY}

- mathematical sciences publishers

nonprofit scientific publishing

http://msp.org/

() 2016 Mathematical Sciences Publishers 


\section{Algebra \& Number Theory}

Volume $10 \quad$ No. $6 \quad 2016$

Modular elliptic curves over real abelian fields and the generalized Fermat equation $x^{2 \ell}+y^{2 m}=z^{p}$

SAMUELE ANNI and SAMIR SIKSEK

Geometry and stability of tautological bundles on Hilbert schemes of points

DAVID STAPLETON

Anabelian geometry and descent obstructions on moduli spaces

Stefan PATRIKIS, José Felipe Voloch and Yuri G. Zarhin

On the local Tamagawa number conjecture for Tate motives over tamely ramified fields JAY DAIGLE and MATTHIAS FLACH

Heegner divisors in generalized Jacobians and traces of singular moduli

JAN HENDRIK BRUINIER and YINGKUN LI

On 2-dimensional 2-adic Galois representations of local and global fields VYTAUTAS PAŠKŪNAS

A probabilistic Tits alternative and probabilistic identities

Michael LARSEN and ANER SHALEV 